\title{
Sensory renal nerves and hypertension
}

Citation for published version (APA):

Janssen, B. J. A. (1988). Sensory renal nerves and hypertension. [Doctoral Thesis, Maastricht University]. Rijksuniversiteit Limburg. https://doi.org/10.26481/dis.19881028bj

Document status and date:

Published: 01/01/1988

DOI:

10.26481/dis.19881028bj

Document Version:

Publisher's PDF, also known as Version of record

\section{Please check the document version of this publication:}

- A submitted manuscript is the version of the article upon submission and before peer-review. There can be important differences between the submitted version and the official published version of record.

People interested in the research are advised to contact the author for the final version of the publication, or visit the DOI to the publisher's website.

- The final author version and the galley proof are versions of the publication after peer review.

- The final published version features the final layout of the paper including the volume, issue and page numbers.

Link to publication

\footnotetext{
General rights rights.

- You may freely distribute the URL identifying the publication in the public portal. please follow below link for the End User Agreement:

www.umlib.nl/taverne-license

Take down policy

If you believe that this document breaches copyright please contact us at:

repository@maastrichtuniversity.nl

providing details and we will investigate your claim.
}

Copyright and moral rights for the publications made accessible in the public portal are retained by the authors and/or other copyright owners and it is a condition of accessing publications that users recognise and abide by the legal requirements associated with these

- Users may download and print one copy of any publication from the public portal for the purpose of private study or research.

- You may not further distribute the material or use it for any profit-making activity or commercial gain

If the publication is distributed under the terms of Article $25 \mathrm{fa}$ of the Dutch Copyright Act, indicated by the "Taverne" license above, 
SENSORY RENAL NERVES AND HYPERTENSION 



\section{SENSORY RENAL NERVES AND HYPERTENSION}

\section{PROEFSCHRIFT}

ter verkrijging van de graad van doctor aan de Rijksuniversiteit Limburg te Maastricht, op gezag van de Rector Magnificus, Prof. dr. F.I.M. Bonke, volgens het besluit van het College van Dekanen, in het openbaar te verdedigen op vrijdag 28 oktober 1988 om 13.30 uur

door

BERNARDUS JACOBUS AGNES JANSSEN

geboren te Sittard 1958 
promotor:

Prof. dr. H.A.J. Struyker Boudier

co-promotor:

Dr. J.F.M. Smits

beoordelingskommissie: Prof. dr. M.A. Allessie

Prof. dr. J. Greven

Prof. dr. R.A. Janknegt

Dr. H.A. Koomans

Prof. dr. S. de Lange

Met dank aan iedereen die een bijdrage heeft geleverd aan het tot stand komen van dit proefschrift.

Experinentele assistentie $H$. van Essen en L. Vervoort-Peters

Sekretariële ondersteuning $\mathbb{E}$. Geurts en $M$. Hogenboom

Omslag

D. Nijhof

Druk

Groenevelt BV, Landgraaf 
voor Dianne

Tiny en Gerrit 


\begin{tabular}{|c|c|}
\hline ADO & adenosine \\
\hline AII & angiotensin II \\
\hline MHOVH & analysis of variance \\
\hline ARN & afterent renal nerve(s) \\
\hline AFMA & afferent renal nerve activity \\
\hline ARN- $x$ & surgical elimination of ARN \\
\hline AVE & arginlne wasopressin \\
\hline bpm & beats per minute \\
\hline BK & bradykinjn \\
\hline BRS & batroreflex sensitivity \\
\hline bew & body wetight \\
\hline 60 & cardlac output \\
\hline DOCA & deoxycort \\
\hline ERN & efferent renal merve(s) \\
\hline $\mathbf{P}$ & furosemide \\
\hline GR & gomerular filtration rate \\
\hline $\mathrm{HP}$ & heart period \\
\hline HOR/HOR & hindquarter flow resistance \\
\hline$H R$ & heart rate \\
\hline $5-\mathrm{HT}^{2}$ & 5-hydroxy tryptamine \\
\hline $1 \mathrm{~m}$ & intramesenteric \\
\hline ip & intraperitoneal \\
\hline Ipv & intrapelvic \\
\hline it & intrarenal \\
\hline iv & Intravenous \\
\hline $1 \mathrm{~K}-1 \mathrm{C}$ & 1 kidney - 1 clip \\
\hline LRF / LRR & left renal flow/resistance \\
\hline MAP & nean arterial pressure \\
\hline MF / MR & mesenteric flow/resistance \\
\hline $\mathrm{NE}$ & norepinephrine \\
\hline NTS & nucleus tractus solitarius \\
\hline PRC & plasma renin concentration \\
\hline RBF & renal blood flow \\
\hline $\mathrm{RF} / \mathrm{RR}$ & renal flow/resistance \\
\hline $\mathrm{RN}-\mathrm{X}$ & complete renal denervation \\
\hline RRF/RRR & right renal flow/resistance \\
\hline SBP & systolic blood pressure \\
\hline SHR & spontaneously hypertensive rate(s \\
\hline $\mathrm{IPR}$ & total perlpheral resistance \\
\hline Uosin & urtnary osmolality \\
\hline UP & ureteral pressure \\
\hline Uv & unimary volume \\
\hline WKV & urinary potassiun excretion \\
\hline UNaV & urinary sodium excretion \\
\hline WK & Wistar-Kyoto \\
\hline
\end{tabular}


Abbreviations

1. Introduction

1.1. General introduction

1.2 The role of the kidney in hypertension

1.3 Renal neuroanatomy 11

1.3.1 Efferent renal nerves 11

1.3.2 Afferent renal nerves 12

1.4 Renal nerves in hypertension 14

1.4.1 Efferent renal nerves 15

1.4 .2 Afferent renall nerves 19

1.5 The present thesis 21

2. Materials and methods

2.1 Animals 22

2.2 Blood pressure measurements 22

2.2.1 Direct intra-arterial measurements 22

2.2.2 Indirect blood pressure measurements 23

2.3 Administration of drugs in conscious
unrestrained rats

2.3.1 Intravenous administration $\quad 24$

2.3.2 Intrarenal adininistration $\quad 24$

2.3.3 Intramesenteric administration 25

2.4 Renal denervation techniques 25

2.4.1 Complete renal denervation 25

2.4 .2 Renal deafferentation 26

2.5 Measurement of baroreflex sensitivity 27

2.6 Measurement of urinary excretion of water and sodium 27

2.7 Measurement of regional blood flow 28

2.8. Electrophysiological recordings of afferent renal
necve activity

2.9 Plasma and tissue analyses 31

2.9.1 Determination of plasma renin concentration 31

2.9.2 Determination of plasma and tissue
norepinephrine concentration

2.10 Data processing and statistical analyses 33

3. Electrophysiological characteristics of chemosensitive afferent renal nerves

3.1 Introduction 34

3.2 Experimental protocols 35

3.3 Results 39

3.4 Discussion 43

3 . 5 Acknowledgement 46

4. Acute hemodynamic effects of activation of renal and mesenteric sensory nerves in conscious rats

4.1 Introduction

4.2 Experimental protocols 47 
5. Hemodynamic effects of prolonged stimulation of afferent renal nerves in conscious rats

5.1 Introduction

5.2 Experimental protocols

5.3 Results

5.4 Discussion

6. Role of afferent renal nerves in renal sodium excretion

6.1 Introduction

6.2 Experimental protocols

6.3 Results

6.4 Discussion

7. Acute hemodynamic effects of diuretics: role for ARN in body fluid volume regulation?

7.1 Introduction

7.2 Experimental protocols

7.4 Discussion

8. Effects of complete renal denerwation and selective afferent renal denervation on the hypertension induced by intrarenal norepinephrine infusions in conscious rats

8.1 Introduction

8.2 Experimental protocols

8.3 Results

8.4 Discussion

9. Role of afferent renal nerves in the development and maintenance of spontaneous hypertension in rats

9.1 Introduction

9.2 Experimental protocols 104

9.3 Results

9.4 Discussion

10. General discussion: the functional role of afferent renall nerves in body fluid homeostasis, cardiovascular control and hypertension

Summary

References 


\section{1 General introduction}

High blood pressure is one of the endemic disorders of the developed Western world, which affects approximately $15 \%$ of all adults. It is one of the leading causes of stroke, congestive heart failure, sudden death and renal failure. Classically, hypertension in man may be divided into primary and secondary forms. More than $90 \%$ of the patients exhibit primary or essential hypertension. The term essential refers to the unknown cause of the disease. The secondary forms include hypertension of established origin such as phaeochromocytoma, primary aldosteronism, chronic glomerulonephritis or renowascular hypertension. However, also in these cases there is disagreement on the mechanisms by which arterial pressure is raised.

Despite a veritable explosion of research over the past few decades, no major cause of the disease has been identified. Considerable advances: have been achieved in our knowledge of the pathophysiology and pathogenesis of arterial hypertension. However, the ever-increasing discovery of new factors involved (e.g. atrial natriuretic peptides, endothelium derived substances) concomitantly raises the number of hypotheses on the aetiology of hypertension. At the same time, this development strengthens the view that no single abnormality of any pressor or antipressor system will emerge as the cause of hypertension. As the complex, schematic diagrams of Guyton and Page have suggested, we will gain more insight by elucidating the multiple interrelations between the various mechanisms. In this respect, the purpose of this thesis is not to introduce the sensory renal nerves as a new factor in the aetiology of hypertension. Rather the ain is to portray these nerves as one of the pathways explaining how the kidneys are interrelated with other systems involved in the regulation of blood pressure.

\subsection{The role of the kidney in hypertension}

The dominance of the kidney in the induction and maintenance of several forns of hypertension has been acknowledged for a long time. Transplantation studies in genetically hypertensive animals (Bianchi et al., 1973 , 1974; Dahl et all, 1974; Kawabe et al., 1979; Rettig et a1., 1988) and

evaluation of data on postransplantation hypertension in man (Guidi et 
al. 1982) have indicated that the development of high blood pressure goes w the kidney. These findings have strengthened the theories on the pathogenesis of hypertension, which are based on the view that abnormal kidney function is essential in the initiation of high blood pressure. The concept that long-term blood pressure is primarily controlled by the intrinsic capacity of the kidney to excrete sodium, was originally developed by Borst and Borst-de Geus (1963). This idea has been elaborated by Guyton and co-workers (1974). Extensive research has revealed that renal excretory function is strongly affected by a number of extrinsic factors. On the other hand, it became evident that blood pressure is to a considerable extent influenced by factors of renal origin. Not only humoral mechanisms are known to be involved. Over the last decade our understanding of neural mechanisms in the control of renal function and blood pressure has advanced remarkably. Many exciting new data hawe become available, concerning the function of the renal nerves.

Already in 1840 (Pappenheim) renal nerves have been identifled as bundles of axons runing along the renal artery and vein into the hilus of the kidney. Later Mitchell (1950) pointed out that in relation to its size, the kidney has a richer innervation than almost any other organ. Nevertheless, until recently, the prevaling view on the function of renal nerves was that they exert no tonic regulatory effect under normal conditions. This view was supported by clinical experience with human transplantation, which necessarily involves denervation of the extrinsic nerves of the transplanted kidney. However, a large number of new experimental data have challenged this interpretation.

The concept of an important role of neural influences on renal excretory function is part of a changing view on the functional role of the ationomic innervation of visceral organs in general. It is becoming clear that integrative neural reflex mechanisms are important in the adaptive responses of nany visceral organs. Hth respect to the renal nerves, it was shown that they do not only conduct intormation from the central nervous system to the kidney. firm evidence has also been obtained for nervous impulses travelling in the opposite direction. Thus, renal nerves must be considered as mixed nerves, containing both efferent (centrifugal) as well as afferent (sensory or centripetal) libers. The contribution of the efferent renal nerves to the control of renal function has been well established. Comprehensive reviews on this subject have recently appeared (DiBona 1982; Gottschalk et al., 1985). The functional significance of the nervous information axising from the kidney remains obscure. Evidence is 
growing for functional role of the sensory renal nerves in the control of renal function and blood pressure. The present thesis is mainly concerned with this concept. For reasons of completeness and understanding of the reflexes arising from the kidney the functions of the efferent as well as afferent renal nerves will be discussed. Their role in the regulation of blood pressure is examined primarily in the context of hypertension.

\subsection{Renal neuro-anatomy}

\subsubsection{Bfferent renal nerves}

There has been extensive discussion on the nature of the efferent renal innerwation. It is commonly accepted that the postganglionic renal. nerves are adrenergic fibers. The presence of cholinesterase containing fibers has been reported (Gosling, 1969; Barajas, 1975). However, functional studies (Zambraski et al., 1978) as well as studies using the specific adrenergic neurotoxin 6-hydroxydopamine (6-0HDA) (Barajas and Wang, 1979) have led to the understanding that the renal inmervation is predominantly adrenergic.

The existence of dopaminergic fibers in the kidney is still a matter of much controversy. The presence of renal dopaminergic innervation may be strongly species dependent (Mc Grath et al., 1983). However, extraneuronal dopamine production by the kidney may have a significant influence on renal function (Struyker-Boudier, 1986).

As has been reported for most visceral organs, it is likely that purimergic and peptidergic nerve fibers are also present in the mammalian kidney (Drukker et al., 1987). Anatomical studies on their existence are, however, scanty (Barajas et al., 1983).

Advanced electron microscopic and histochemical studies have shown adrenergic nerve terminals in association with renal blood vessels, yuxtaglomerular cells, as well as basement membranes of both proximal and distal tubules (Barajas, 1973, 1978). In a more recent quantitative study, Barajas et al. (1984) found the highest density of nerve endings in association with the thick ascending limb of Henle's loop, followed by the distal convoluted tubule and the proximal tubules. These anatomical data are in good correlation with the results from functional studies. As depicted in table 1.1 efferent renal nerves are implicated in a number of processes that relate to blood pressure control. There is an abundance of evidence that upon activation, they stimulate sodium reabsorption and renin release and 
Table 1.1. Adrenoceptor type and functional correlation in different parts of the kidney

\begin{tabular}{|c|c|c|}
\hline Structure & Receptor type & Functional correlation \\
\hline Arteries & Alpha-1 & Vasoconstriction \\
\hline Juxtaglomerular cells & $\begin{array}{l}\text { Beta-1 } \\
\text { Alpha-1 } \\
\text { Alpha-2 }\end{array}$ & $\begin{array}{l}\text { Stimulation renin release } \\
\text { Stimulation renin release } \\
\text { Inhibition renin release }\end{array}$ \\
\hline Proximal tubules & $\begin{array}{l}\text { Alpha-1 } \\
\text { Alpha-2 }\end{array}$ & $\begin{array}{l}\text { Stimulation sodium reab- } \\
\text { sorption } \\
\text { Inhibition sodium reab- } \\
\text { sorption }\end{array}$ \\
\hline Distal tubules & Beta-1 & $\begin{array}{l}\text { Stimulation sodium reab- } \\
\text { sorption }\end{array}$ \\
\hline
\end{tabular}

lead to vasoconstriction hereby reducing glomerular filtration. Detailed information on adrenoceptor localization and pharmacology and functional correlation have recently been reviewed extensively (DiBona, 1982, 1988; struyker-Boudier et al., 1987).

\subsubsection{Afferent renal nerves}

The morphology and localization of afferent renal nerve (ARN) endings within the kidney is still an unresolved matter. No clear histologic demonstration of thelr presence has been reported. Their existence, however, is undisputed on the basis of electrophysiological studies (Moss, 1985). It is probable that the ARN endings are undifferentiated dendrites, which, using light microscopy, would be indistinguishable from efferent endings. Barajas and Vang (1978) have suggested that myelinated fibers with the renal cortex are afferent. Notley (1968) suggested that nerve terminals in the mucosa and submucosa of the renal pelvis and ureteropelvic junction are of afferent nature. Several electrophysiological studies have supported this latter idea (Recordat et al., 1978; Katholl et al., 1983).

The projections of ARN to dorsal root ganglia and into the spinal cord have been studied in the rat (Lappe et al., 1982; Cirlello and Calaresu, 1983; Donovan et a1.. 1983; Gattone et al., 1986) and cat (Kuo et al., 1983) using the antegrade transport of horseradish peroxidase or the fluorescent dye True Blue. In the rat ARN from the left kidney enter the spinal cord ipsilaterally through several dorsal root ganglia at the level of 
T8 through $L 2$. The distribution of ARN cell bodies in the dorsal root ganglia are positioned cranially (T6-Li) in relation to those from the right kidney. In the rat only ipsilateral projectlons have been identified, whereas in the cat there is also a slight degree of contralateral projections (Kuo et al., 1983). ARN cell bodies in the dorsal root ganglia are of small and medium size (Ciriello and Calaresu, 1983; Kuo et al., 1983) sugm gesting that most of the ARN fibers are small unmelinated C-fibers or thin myelinated Af fibers. Electrophysiological studies support this suggestion (Niljima, 1975; Calaresu et al., 1978). In addition to the spinal cord projections, ARM cell bodies have been found in the nodose ganglia in the rat (Ciriello and Calaresu, 1982; Gattone et al., 1986). In the paper of Gattone and co-workers more neurons were labelled in the nodose ganglia than in the combined dorsal root ganglia, suggesting that most of the renal sensory innervation might be of vagal origin. Functional or electrophysiological evidence for this hypothesis is, however, not avallable. Furthermore, it should be considered that the kidney could possess intramural sensory ganglia as has been reported for most of the visceral organs (Drukker et al., 1987). However, this possibility has never been investigated systematically. Unless stated otherwise, all further reports in this thesis deal with the ARN ruming via the spinal cord (sympathetic afferents)."

The projections of ARN have been traced with electrophysiological studies in cats (Ciriello and Calaresu, 1980; Calaresu and Ciriello, 1981; Ciriello and Caverson, 1987; Caverson and Ciriello, 1988) and in rats (Simon and Schramm, 1984; Rnuepfer et al., 1985; Hebb and Brody, 1987). In the brainstem, both the nuclei of the solitary tracts (NTS) (Simon and Schramm, 1984) and the lateral parabrachial nuclei seem to relay ARN information. Selective lesioning of either of these nuclei abolishes the hemodynamic responses to ARN stimulation (Webb and Brody, 1987). Interestingly, the NTS is also the major brainstem nucleus relaying arterlal baroreceptor 1 formation. Recently, it has been shown that ARN inputs to cells in the region of the NTS can inhibit the response of these cells to carotid sinus and aortic depressor nerve stimulation (Felder, 1986). This finding suggests the existence of a mechanism by which ARN may alter the flow of sensory information from the circulatory system into the central nervous system.

Projections of ARN to sites located in the forebrain are extensively described by Ciriello and Calaresu (1980), Ciriello and Caverson (1987), and Webb and Brody (1987). Electrical stimulation of ARN was found to elicit responses from the lateral hypothalamus, lateral and periventricular pre-optic region and paraventricular nucleus. Recent experiments by Caver- 
son and ciriello (1986) have shown that in cats electrical stimulation of ARH may excite meurosecretory neurons in the paraventricular nucleus leading to an increased vasopressin release. These findings indicate that the ARN are connected to reglons in the central nervous system that are known to be inwolwed in cardiovascular control.

\subsection{Renal nerves in hypertension}

The important contribution of the neural control of renal function in hypertension is best reflected by examining the effects of renal denervation in experimental animal models of hypertension. Table 1.2 summarizes the models in which renal demervation delays or prevents the development of hypertension. Note that in these experiments renal denervation was accomplished by stripping the renal artery and vein, whereafter the vessels were painted with phenol. Since no sellective denervation of ARN or efferent renal nerves (ERN) was performed (we shall further refer to this method as

Table 1.2. Models of experimental hypertension in which renal denervation delays or prewents the development of hypertension

Spontaneously hypertensive rat Spontaneously hypertensiwe rat Spontaneously hypertensive rat Spontaneously hypertensive $\mathrm{rat}$ Spontaneously hypertensive rat Spontaneously hypertensive rat SHR stroke prone

New Zealand SHR

Goldblatt 1-kidney, $1-\mathrm{c}$ lip rat Goldblatt 1-kidney, 1-clip rat Goldblatt 2-kidney, 1-clip rat Aortic coarctation dog Aortic nerve transsection rat DoCA/sall rat DoCA/sall rat DoCA/salt rat Grollman renal wrap rat Grollman renal wrap rabbit Low-sodium, 1 -kidney hypertension rat Angiotensin-1induced hypertension rat

$\begin{array}{ll}\text { Liard } & 1977 \\ \text { Kline et al. } & 1978 \\ \text { Winternitz et al. } & 1980 \\ \text { Säynäälammi et al. } & 1982 \\ \text { Norman and Dzielak } & 1982 \\ \text { Takeda et al. } & 1987 \\ \text { Nagaoka and Kakihana } & 1982 \\ \text { Dizet al. } & 1982 \\ \text { Katholi et al. } & 1981 \\ \text { Norman et al. } & 1984 \\ \text { Katholl et al. } & 1982 \\ \text { Whitlow and Katholi } & 1982 \\ \text { Kline et al. } & 1983 \\ \text { Katholi et al. } & 1980 \\ \text { Takahashi et al. } & 1984 \\ \text { Takeda et al. } & 1987 \\ \text { Fink and Brody } & 1980 \\ \text { Kline et al. } & 1986 \\ \text { Vari et al. } & 1986 \\ \text { Vari et al. } & 1987\end{array}$


complete renal denervation), no conclusion can be drawn as to whether the hypotensive effect of complete renal denervation is the result of the loss of the ERN, ARN or both fiber populations. Selective denervation techniques for the ERN (Le Noble et al., 1985) and ARN (Lappe et al., 1985) have recently become available. As for the functional anatony, we shall discuss the evidence for the involvement of the ERN and ARN nerves in hypertension separately.

\subsubsection{Efferent renal nerves}

From the above mentioned functional characteristics of the ERN (table 1.1), it is obvious that an increase in their activity may contribute to the induction of hypertension. ERN activity has been assessed in a variety of rays in several models for the disease as well as in patients. The most indirect measure is probably the renal concentration of norepinephrine (NE) as determined either chemically or by histofluorescent methods. Also the turn-over rate of $\mathrm{NE}$ has been used to estimate nerve activity. This approach, where the disappearance of NE after blockade of its synthesis is measured, probably reflects nerve activity more accurately. As yet another indirect method, renal spill-over of $\mathrm{NE}$ into the circulation may be used to estimate sympathetic activity. In the latter case it has been shown that renal NE release as calculated from renal flow and veno-arterial concentration differences in dogs does indeed parallel sympathetic renal nerve activity (Oliver et all, 1980; Kopp et al., 1983). All of the before mentioned methods have the same limitation, $i, e$. the process studied is not only dependent upon nerve activity, but also on storage and release mechanisms. When studying hypertensive subjects it should be noted that these mechanisms may be disturbed in some way (Westfall et al., 1985). Taking these considerations into account we have to conclude that direct recording of ERN activity is probably the most reliable index of nervous activity.

Renal NE concentrations were measured in several renal hypertensive animal models. Results indicate that renal NE is either reduced (Fink and Brody, 1978; Villarreal et al., 1984) or unchanged (kline et al., 1986) in established renal hypertension. Also in DOCA-NaCl hypertension conflicting results have been obtained. In this model for hypertension, elther decreased (Kline, 1982; Fujita and Sato, 1984) or unchanged (Katholi and Haftilan, 1980; Takahashd et al., 1984) NE levels were found. Measurement of turn-over rate showed increased turn-over associated with reduced tissue levels in one study (Fujita and Sato, 1984). Recently, direct recording of multi-fiber ERN activity revealed no differences in resting activities in 
DOCA hypertensive and sham treated animals, although the excitability of ERN was strongly increased in hypertenslve animals (Koepke et al., 1986). Based on denervation studies it has been argued that renal nerves might be Involved in the initiation of this form of hypertension although much less In Its malntenance (Katholi, 1985, Katholi et al., 1983). Other factors such as the timing of unilateral nephrectomy have been suggested to imfluence the outcome of studies wth regard to the contribution of the renal nerve activity (Katholl et al., 1983; Dzielak and Norman, 1985). The reason for the discrepancy of these results remains unresolved.

In genetic models for hypertension such as the Okamoto SHR renal $\mathrm{NE}$ content was found to be slightly elevatted in adult animals (Norman and Dzlelak, 1982). Measurements of turn-over rate indicates an increased $\mathbb{N E}$ turn-over in young but not in adult animals (Yamori, 1972). Thus, also in this species renal merves might be involved more in the initiation of hypertension than in 1 ts maintenance, which is again in accordance with findings in denervation studies (see above). In contrast, most studies in which ERN activity was measured as single- (Lundin et al., 1984) or multifiber activity (Lundin et al., 1984; Thorén, 1987) indicate increased resting activity in conscious and anesthetized SHR as compared to normotensive control animals. Also excitability to stimuli as baroreceptor loading and unloading, low pressure receptor stimulation and mental stress was increased. In one study, only excitability was increased, whereas resting activity was not (Francisco et al., 1981). Schram and Chornoboy (1982) suggested on the basis of spinal transsection studies that the elevation of resting tone in SHR was of spinal origin, whereas the spinal renal interconnection had normal excitability.

In patients with essential hypertension, renal spill-over of NE has been compared to that of normotensive controls. In a recent review paper, Ester et al. (1988) show that in young hypertensive patients renal $N$ spill-over is increased, whereas in those with established hypertension it returns to normal. Also, in patients with essential hypertension ERN excitability has been suggested to be increased (De Leeuw et a1., 1987). Thuis, in human essential hypertension ERN also appear to be involved in the inim tiation of hypertension.

It should be added that increased renal nerve activity must lead to increased activity of effector mechanisms. One of the processes through which the body may adapt to increased nervous tone is down-regulation of receptors, in this case renal alpha- or beta-adrenoceptors. The reverse of this process (i.e. up-regulation of receptor numbers following renal surgi- 
cal denervation) has been shown to occur in rats (KIIne et Mercer, 1980). Not only did those authors show increased receptor number, but also an increased responsiveness of the renal circulation to exogenous NE (KIine and Mercer, 1980). This latter effect does not seem to be dependent on increased receptor numbers, since recently denervated rat kidneys were shown to be hyperresponsive to a number of non-adrenergic vasoconstrictor stimuld as well. In renal (Högestät et al., 1987) and DoCA-NaCl hypertensive rats, unchanged densities of renal alpha-1 and alpha-2 receptors were found (Pettinger et al., 1982). In contrast, in okamoto SHR (Sanchez and Pettinger, 1981; Pettinger et al., 1982) stroke-prone SHR (Yamada et a1., 1986) and Dahl-S hypertensive rats (Pettinger et al., 1982), both renal alpha-adrenoceptor subtypes were elevated as compared to those in their control animals. Others have also found increases in renal beta-adrenoceptor densities in SHR and normotensive rats. Both in normal SHR (Struyker-Boudier et al., 1985) and strokemprone SHR (Yamada et al., 1982) increased numbers of renal beta-adrenoceptors have been reported. Fractionation studies revealed that this increase in receptor number could be ascribed to increased tubular receptor density (Struyker-Boudier et al., 1985) as has also been suggested for alpha-adrenoceptors. Thus, contrary to what might be expected, the increased sympathetic nerve activity that has been observed, does not lead to down-regulation of receptors. In fact, in genetic hypertension the "Inappropriate" reaction of the receptors to increased stimulation has been suggested as one of the possible mechanisms leading to hypertension. In a recent study, however, the functional significance of the increased receptor density for alpha-2 receptors in rats was challenged (DiBona and Sawin, 1987), because neither renal vascular nor tubular responses to nerve stimulation and adrenergic receptor agonists in SHR were changed as compared to those in normotensive animals.

Considering all the evidence, at least in genetically hypertensive animals and in human essential hypertension and possibly also in mineralocorticoid and renal hypertensive animals, there is good evidence for increased renal nerwe activity. Furthermore, renal adrenoceptors do not seem to compensate for the increased activity. However, the question whether there is a causal relationship between ERN activity and hypertension cannot be answered on the basis of the above mentioned studies. To study this hypothesis, Kottke et al. (1945) stimulated renal and splanchnic nervem chronically in dogs. This resulted in sustained hypertension. Another approach was chosen by Kathold and comorkers (1977), who were the first to chronically infuse $\mathrm{NE}$ into the renal artery of dogs. They were also capable 
of Inducing sustained hypertension. Intravenous infusion of similar amounts of NE caused only a transient rise in blood pressure. Later studies by others in dogs (Cowley and Lohmeler, 1979; Lohmeier et al., 1984) and from our own laboratory $\mathbb{1 n}$ rats (Kleinjans et al., 1983; Kleinjans et al., 1984a; Klelinjans t al., 1984b) confirmed the finding of a sustained increase in blood pressure during intrarenal infusion of NE in doses that were ineffective or much less effective when given intravenously. Thus, the increased ERN activity that has been observed in hypertension may indeed be causal to the hypertension. What we should be able to learn from these studies is the (intrarenal) effector mechanism that is triggered by the increased nerve activity. The studies that have been performed in dogs emplloyed a fixed sodium intake. Under these conditions Katholi et al. (1977) and Lohmeier et a1. (1984) observed a positive sodium balance during intrarenal infusion of $N E$. During intravenous infusions in dogs, only transient blood pressure increases and a negative sodium balance were observed.

These observations are compatible with the hypothesis of Borst and Borst-de Geus (1963) and Guytion et al. (1974) that changes in the relationship between blood pressure and urine output can lead to hypertension through retention of sodium and water and consequent increases in cardiac output and, in a later stage, total peripheral resistance. Our own observations in rats, however, indicate that this is not a universal mechanism. In a study where we allowed rats free access to sodium and water, we noted a decreased sodium excretion which was, however, compensated by a decreased sodium intake (Kleinjans 1984b). Further detailed hemodynamic studies revealed that at no time there was evidence for an increased cardiac output, but that the total peripheral resistance increased from the start of the infusion (Kleinjans et al., 1984b). Thus, at least in rats other mechanisms must be involved. In studies in dogs (Katholi et al., 1977; Cowley and Lohmeler, 1979; Lohmeler et al., 1984) an increase in plasma renin activity was noted and although this was suggested to contribute to the hypertension (Cowley and Lohmeler, 1979; Lohmeier et al., 1984), this could not be conEirmed by others in the same species (Katholi et al, 1977) and in rats (Kleinjans, 1983). In these latter studies blockade of the renin angiotensin system could not ptevent the exaggerated increase in blood pressure during intrarenal as compared to intravenous infusions. Thus, hypertension during simulated increased efferent renal sympathetic stimulation does not laad to hypertension following the routes that might be expected on the basis of the hypothesis of Borst and Borst-de Geus (1963).

The most striking observation that was made by Katholi and co-workers 
and for wich we also obtained evidence, is that the hypertenston during intrarenal $\mathrm{NE}$ infusion is associated with generalized sympathetic activation. In their dogs, Katholi et al. (1977) vere capable of normalizing total peripheral resistance with the alpha-adrenoceptor blocking agent phentolamine. Similarly, measurement of NE plasma levels during intrarenal and intravenous infusions of $\mathrm{NE}$ revealed much higher systemic NE levels during infusion via the former route than during infusion of the same doses via the latter route (Kleinjans et al., 1984b). This effect did not depend upon decreased clearance as determined by tracer infusion studies (Kleinjans, 1983). The latter observations would suggest increased release of NE from nerve terminals, either because of increased firing of sympathetic nerves, or from interaction of, for instance, angtotensin II with presynaptic release mechanisms. Alternatively, afferent nerves, originating from the kidneys, might be triggered by the NE infusions in a manner, similar to that observed for the sino-aortic baroreceptor fibers (Mills and Smith, 1983). These mechanisms and their possible contribution to hypertension vill be discussed below.

\subsubsection{Afferent renal nerves}

The existence of ARN has been recognized for many years, not in the least as the origin of renal pain sensation (De Wolf and Fraley, 1975). Renal denervation has been advocated by some as a method to allewlate chronic pain of renal orlgin (Harris and Harris, 1930; Laughton, 1972). In recent years several distinct types of renal sensory receptors have been identified. They can be broadly divided into renal mechanoreceptors and renal chemoreceptors. Renal mechanoreceptors respond to increases in arterial, venous, tissue and ureteral pressure in a variety of species. Renal. chemoreceptors are sensittve to chemical alterations in the intrarenal environment. Detalled descriptions of their electrophysiological characteristics are given in chapter 3 of this thesis and in a recent review by Moss (1985). It is argued that these units are tonically active under normal physiological conditions. Their activity may give rise to a wariety of reflexes which are involved in the regulation of renal function and blood presisure.

The existence of such reflexes is known from numerous reports which show that a change in excretory activity in one kidney is quickly followed by a reciprocal change in the excretory activity in the other kidney, such that total excretions of salt and water are unchanged or minimally affected. This may occur following unilateral occlusion of a renal artery or 
win or a weter and after unilateral denervation. The characteristics of these so-called renorenal reflexes have been described in detail by Kopp (1985) and Gottschalk et al. (1985).

When surveying the literature, there are striking contradictions in the findings w th regard to the acute effects of ARN stimulation on hemodynamics. Arterial pressure has been observed to decrease during ARN stimulation in the rat (Aars and Akre, 1970; Vebb and Brody, 1987) whereas in other reports opposite responses were found (Hermansson et al., 1984). Similar conflicting results have been reported for other species (Beacham and Kunze, 1969; Ueda et al., 1967; Callaresu et al., 1978). Such variations may reflect specles differences, anesthetic effects, as well as variation of the experimental preparations. In addition, since ARN consist of a heterogeneous fiber population, the contradictions may well be the result of activation of different fiber populations in those experiments. Only a few studies have been performed in conscious animals. Stimulation of ARN in conscious animals was achieved by intrarenal infusions of bradykinin (Smits and Brody, 1984) or adenosine (Katholi and Woods, 1987) and by reducing renal blood flow (Faber and Brody, 1985). These interventions caused pronounced pressor responses which could be prevented by anesthesia, ganglionic blockade, complete ipsilateral denervation or a selective surgical interruption of the ARN. Thus, these acute increases in blood pressure depend upon activation of the efferent sympathetic nervous system through ARN.

As sha1 be discussed thoroughly in this thesis, it becomes important to determine to what extent the ARN dependent sympathetic pressor mechanisms cam contribute to the inititation and maintenance of hypertension. Indications for the involvement of ARN in some models of experimental hypertension may be deduced from the fact that in a number of models, sumarlzed in table 1.2 , the decrease it blood pressure after complete renal denervation was associated with a reduction of sympathetic nervous activity. Cardiovascular responses to ganglionic blockade or plasma NE levels returned to values as observed in normotensive controls (Winternitz et al., 1980, 1982; Săynăvălami et al., 1982; Katholi et a1., 1982a, 1982b, whitLow and Katholï, 1982; Krueger et al., 1986; Takeda et al., 1987). Also changes in hypothalanic NE content (binternitz et al., 1982b) and cardiovascular responses to electrical stimulation of hypothalamic structures (Takeda et al., 1987) have been reported after complete renal denervation. This suggests that peripheral section of these nerves can alter central sympathetic tone. The alterations in sympathetic tone may be explained on 
the basis of changes in remal humoral factors, resulting from the loss of efferent renal sympathetic nerwe activity. Alternatively, it might depend upon a reduction of ARN input to spinal or central structures which integrate renal sensory information in the sympathetic nervous activity (see abowe). Selective renal deafferentation studies are necessary to evaluate this hypothesis.

In two animal models of hypertension " such studies have been performed. In Grollmann renal wrap hypertensive rats, renal deafferentation prior to wrapping could not prevent the rise in blood pressure in these rats (Pan et al., 1985). Renal deafferentation prior to clipping of 1-kidney, rats did lower blood pressure significantly in comparison with clipped intact rats. Blood pressure was, however, still elevated above levels in non-clipped control rats (Wyss et al., 1986). Thus, in this model hypertension was attenuated rather than prevented by this surgical intervention.

\subsection{The present thesis}

Classically the kidney is thought to play a key role in the regulation of body fluid homeostasis and blood pressure by controlling the excretion of water and electrolytes. The contribution of neural mechanisms to these processes has been recognized only recently. Studies in this field were mainly concerned with the functional significance of the efferent renal nerves. Little attention has, however, been paid to the afferent renal nerves. Although the first afferent renal nerve recordings were made some 25 years ago, the concept that the kidney is an important sensor organ in the control of body fluid homeostasis and blood pressure has not gained much support. The fact that most of the experinents were performed under stressfull conditions such as anesthesia, surgery or trauma, may have contrillbuted to the view that ARN induced changes in renal excretory function or blood pressure were secondary to changes in renal hemodynamics. Now that techniques have come available to study the functional significance of ARN in conscious unrestrained animals, this interpretation has been challenged. To contribute to the changing point of view in this mater, it is the alm of the thesis to elucidate this concept and to present evidence which supports the hypothesis that the kidney is an important sensor organ, which, vila the ARN, modulates mechanisms that play key role in the regulation of body fluid homeostasis and blood pressure. 


\subsection{Andmals}

In all studies, experiments were performed on wale rats unless fndicated otherwise. Depending upon the experimental protocols, rats derived from different strains were used. In the studies presented in chapter 4, 6 , 7 , and 8, normotensive Wistar rats (Winkelmann, Borchen, Germany), were used. The experiments described in chapter 3 were performed on normotensive Sprague-Daley rats (Charles River Breeding laboratories, Raleigh, NC, USA). Spontameously hypertensive rats (SHR) and normotensive wistar-Kyoto rats (WKY) (chapter 5 and 9) were obtained from the breeding colonies of the Central Animal Facilities of our uniwersity. Originally, these rats were derived from the Okamoto-Wistar strains (Okamoto and Aoki, 1963).

During most experiments the rats were housed separately in standard laboratory cages. The animals had free access to standard food (Hope farms, type RMM-TM, Hoerden. The Netherlands) and tap water. In two studies (chapter 7 and 9) it was necessary to house animalis in metabolism cages (type 1760, Techniplast, Buggugiate, Italy). Before these experiments were started, at least 4 days of habituation were allowed. In one experiment rats ate a sodium-free food prepared by Hope farms (Woerden, The Netherlands). This food was found to contain less than $0.03 \% \mathrm{Na}^{*}$, as determined by flame photometry (Eppendorf GmbH, Hamburg, Germany). In several studies it was necessary to perform experiments on animals with one kidney only. In these cases, a nephrectomy (left-sided) was performed wia a paravertebral approach to allow compensatory hypertrophy at least 4 weeks before entering the study .

\subsection{Blood pressure measurements}

\subsubsection{Direct intra-arterial measurements}

The technique for chronic measurement of blood pressure in conscious unrestrained rats has been extensively described by Snits (1980). Briefly, a catheter was constructed from a $9-\mathrm{cm}$ piece of $\mathrm{PE}-10$ tubing, heat-sealed to a 12-cm piece of PE-50 tubing. Two small rims were made at the connection to facilitate securing of the catheter. On the other end of the PE-50 
tubing, a 1-cm piece of PE-100 tubing was heat-sealed and a 5-cm piece of vinyl tubing (Serva TT 63) slid over it. Finally, the PE-10 tubing was bent into a $J$-shape by dipping it into near-bolling vater. This catheter was linplanted in the abdominal aorta by an approach via either the left or right femoral artery. Under light ether anesthesia a small incision was made in the groin and the femoral artery was freed from connective tissue. After the artery was clamped with silk suture, a small hole was cut in 1 it and the $P E-10$ catheter inserted. It was advanced for $4 \mathrm{~cm}$ into the artery so that its tip was approximately $1 \mathrm{~cm}$ above the bifurcation and below the renal arteries. The catheter was secured to the artery and to the underlying muscle with silk and guided subcutaneously to the neck where it was anchored and exteriorized. The catheter was filled with heparinized $0.9 \%$ NaCl (5 $\mathrm{IU} / \mathrm{ml}$ ) and closed with a metal plug. The wounds were sutured with $2-0$ sllk.

For daily blood pressure measurements, the rats were placed in plastic experimental cages $(22 \times 13 \times 13 \mathrm{~cm})$ and the catheter was connected to a low-volume pressure transducer (type CP 01, Century Technology Company, Inglewood, Ca, USA). The transducers were calibrated daily using a mercury manometer. The signal was recorded on a Grass model 7D, polygraph (Grass Instruments, Quincy, Mass, USA) or on a Schwarzer recorder (models RS 348 or US 266, Schwarzer, GmbH, München, Germany). Mean arterial pressure (MAP) was obtained by low-pass filtering $(0.5 \mathrm{~Hz})$ of the pulsatile blood pressure signal. Heart rate (HR) was determined from the pulsatile signal by a tachograph (Grass polygraph or Narco type 7302 (Narco Bio-systems, Houston, Tx, USA). The rats were allowed to habituate for at least 30 min before recording the hemodynamic variables.

\subsubsection{Indirect measurements}

In one experiment (chapter 9) it was necessary to obtain data on the blood pressure levels of the rats over a 3 -months period. Therefore, blood pressures were determined via a non-invasive method (sphygmomanometric tal1-cuff method). A programmed electrosphygmonanometer (Narco Bio-systens PE-300) equipped with a piezo electric pulse pick-up and a band pass filter (3-10 $\mathrm{Hz})$ to increase sensitivity of the method, were used. Rats vere placed in special restrainers and the temperature was maintained at $38^{\circ}-39^{\circ} \mathrm{C}$ to obtain adequate vasodilation of the caudal artery. The electrosphygmomanometer was inflated at a rate of $10 \mathrm{~mm} \mathrm{Hg} / \mathrm{s}$ and the systolic blood pressure was determined on the basis of at least 3 measurements. Heart rate was obtained via a biotachometer (Narco) from the pulsatile blood pressure signal. 
For several experiments, it was necessary to administer drugs via different routes in conscious unrestrained rats " when drugs were applied systemically, a venous catheter was placed in the caval vein, wia cannulation of the femoral vein. For local delivery of drugs to specific target organs such as the kidney or the mesentery. a side-branch of the respective main feeding atery was cannulated as described below.

\subsubsection{Intravenous administration}

The wenous catheter was constructed of a $9-\mathrm{cm}$ piece of $\mathrm{PE}-10$ tubing, heat-sealed to a $15-\mathrm{cm}$ plece of $P E-50$ tubing. Two small rims were made at the connection and at a point $12 \mathrm{~cm}$ further on the PE-50 tubing. Under Iight ether anesthesia, a small incision was made either in the right or left groin of the rat. After the femoral vein was carefully dissected free from the surcounding tissue and clamped with silk sutures, the end of the catheter was inserted through a small hole and advanced for $4 \mathrm{~cm}$ into the wena cawa. The catheter was secured with silk and exteriorized in the neck in the same way as described for the abdominal aorta catheter. The catheter was filled with a $0.9 \% \mathrm{NaCl}$ solution.

\subsubsection{Intrarenal administration}

The technique for local infusion into the renal artery of unrestrained rats has been described in detail elsewhere (Smits et al., 1983). The catheter was constructed of a 15 -cm piece of PE-10 tubing. The tip of the catheter had been stretched over a hot soldering iron to reduce its outer diameter to approximately $0.2 \mathrm{~mm}$. Two rims were made $9 \mathrm{~cm}$ from the tip of the catheter to facilitate securing. A 6-cm piece of $\mathrm{PE}-60$ tubing was heatsealed to the other end of the catheter. In near-bolling water, the catheter wis bent into a coll to prevent its obstruction after attaching the subcutaneous minipump (see below). For implantation of the catheter, the rats were anesthetized with ether. An abdominal midline incision was made and the Intestines were kept in gauzes, wetted with $0.9 \% \mathrm{NaCl}$. The suprarenal artery was dissected free from the surrounding tissue. Care was taken to minimize surgical trauma to the branches of the renal nerwes which run in parallel with the suprarenal artery. The suprarenal artery was clamped with $6-0$ silk and the catheter was carefully inserted through a small hole cut with iris scissors, until its tip reached the bifurcation with the renal artery. The catheter was secured to the vessel and guided subcutaneous- 
Iy to the neck where it was connected to an osmotic minipump (Alzet ${ }^{\text {, }}$, type 2001, Alza Corporation, Palo Alto, Ca, USA) to maintain patency of the catheter. The pumps were primed in saline at $37^{\circ} \mathrm{C}$ for 4 hours before inplantation and filled with $0.9 \%$ NaCl (pumping rate: 1 HIhr). The pumps were implanted subcutaneously between the shoulder blades and all wounds were closed in layers ith silk. Rats were allowed to recover for at least $2-3$ days.

The day before the experiments were started, the catheter was exteriorized and filled th heparinized saline. This method has been shown to leave renal function fully intact (Smits et al., 1983).

\subsubsection{Intramesenteric administration}

To apply drugs directly into the blood stream of the mesentery of a conscious unrestrained rat, a method basically the same as for intrarenal administration of drugs was developed.

Under ether anesthesia, an identical catheter as for intrarenal infusions was implanted via a small upper mid-abdominal incision. The intestines were carefully spread and kept in gauzes wetted with $0.9 \% \mathrm{NaCl}$. After the superior mesenteric artery had been identified, one of the first small side-branches was dissected free. The vessel was clamped with silk sutures (6-0) and a small hole was cut in it with iris scissors. Then the thin end of a PE-10 catheter was inserted retrogradely into the vessel without obstructing blood flow in the main artery. The catheter was fixed to the vessel and muscle wall of the gut and guided subcutaneously to the neck, where $i t$ was connected to an osmotic minipump as described above. After the wounds were closed the rats were allowed to recover for at least $2-3$ days. The day before the experiments started, the catheter was exteriorized and filled with heparinized saline under light ether anesthesia. At the end of the experiments the catheter was flushed with 200 wi indian ink or a methylene blue solution, to verify its location.

\subsection{Renal denervation techniques}

\subsubsection{Complete renal denervation}

Rats were anesthetized with sodium pentobarbital (Brocacef, Heerlen, The Netherlands; $60 \mathrm{mg} / \mathrm{kg}$ body weight, $1 . p$.$) . The kidney was exposed re-$ troperitoneally through a small paravertebral incision and isolated from its surrounding tissue. Denervation was accomplished by strippling the renal 
artery and vein ith fine forceps, followed by application of a $10 \%$ (w/v) phenol in alcohol solution on the renal vessels. For sham denervation the same surgical procedure was followed, but the nerve bundles were left intact and the vessels were painted with normal saline. The wound was closed in layers with 2-0 silk and the rats were allowed to recover for 7-8 days before further surgery. Verlfication of complete renal denervation was done by measuring renal norepinephrine content as described below.

\subsubsection{Renal deafferentation}

Rlimination of afferent renal nerves (ARN-x) from the right kidney was performed by unilateral dorsal rhizotony as described by Lappe et al. (1985). The surgery was performed under aseptic conditions. Under sodium pentabarbital anesthesia, a dorsal longitudinal midline incision was made to expose the thoracic vertebrae T9-T13. Connective tissue and muscle were carefully removed to gain access to the dorsal surface of the vertebrae. Under microscopic view small holes were made in the right vertebral laminae of the vertebrae T8-T12, using a fine bore drill (Carlo di Giorgi, type EM 2000, Milano, Italy). Through these openings, the dorsal roots that have their entry at T9-T13 and occasionally L1, can be reached. ARN from the right kidney have been found to project ipsilaterally through the dorsal roots $\mathrm{T} 7-\mathrm{L} 1$, but most fibers $(90 \%)$ are confined to the dorsal roots T9-T13 (Lappe et al., 1982; Donovan et al., 1983). A small hook was used to lift the dura, which was then cur with iris scissors. In the same way, the arachnoidea was cut. The dorsal roots T9-T13 were lifted with fine U-shaped glass rods and an approximately 3 mm segment was cut out from them. Then, the right vertebral laminae were covered with gel foam (Spongostan, Ferrosan, Denmark). The muscle and skin were sutured separately. For a sham renal deafferentation, the same surgical procedures, except for the actual thizotomy, were followed. After the surgery, each rat received $0.2 \mathrm{ml}$ chloramphenicol (25\% W/V; A.U.V., Cuyk, The Metherlands) subcutaneously and was allowed to recover for $7-10$ days.

At the end of the experiments, animals were flushed transcardially wh $10 \%$ formaline. To verify renal deafferentation, the spinal cord was dissected and visually inspected under a dissection microscope. Only the animals in which a complete transsection of the dorsal roots T9-T13 was observed were included in the data as being deafferented. In the sham operated rats all dorsal roots vere intact. 
Baroreflex sensitivity was determined as described by Smits et al. (1987). As an index for the sensitivity of the baroreflex, the ratio was calculated between changes in heart period (HP 60,000/heart rate) and changes in mean arterial pressure (MAP) induced by intravenous bolus injections $(50 \mu 1)$ of several concentrations of phenylephrine (Sigma) and sodium nitroprusside (Boffmann-La Roche, Mijdrecht, the Netherlands) administered in random order. At least 9 different depressor and 9 different pressor responses were induced, ranging between -50 to 0 and 0 to $+60 \mathrm{~mm} \mathrm{Hg} \mathrm{respec-}$ tively. Only linear regression lines with a correlation coefficient greater than 0.8 and a P-value less than 0.05 were included into the data.

\subsection{Measurement of urinary excretion of water and sodium}

To measure effects of renal deafferentation during reduced sodium intake on urinary excretion of water and sodium in conscious unrestrained rats (chapter 6), intake of water and sodium had to be carefully controlled. According to Struyker-Boudier (1981) this was done by delivering a constant amount of water and sodium by intravenous infusion of a Ringer's solution. This solution contained $154 \mathrm{mM} \mathrm{NaCl}, 2.7 \mathrm{mM} \mathrm{KCl}$ and $1.8 \mathrm{~mm} \mathrm{CaCl} 2$ per liter pyrogen-free distilled water. The $\mathrm{pH}$ was adjusted to 7.4 with a $0.1 \mathrm{mM} \mathrm{NaH} 2 \mathrm{PO}_{4} / \mathrm{Na}_{2} \mathrm{HPO}_{4}$ buffer. During the period of reduced sodium intake, the Ringer's solution was replaced by a similar solution containing $15.4 \mathrm{mM}$ choline Cl instead of $154 \mathrm{mM} \mathrm{NaCl}$. The Ringer's infusion was started $48 \mathrm{hr}$ before experiments began at a constant rate of $1.25 \mathrm{ml} / \mathrm{hr}$. This resulted in a fluld intake of $30 \mathrm{ml} /$ day and sodium intake of 4.7 meq/ day which $1 \mathrm{~s}$ equivalent to the sodium content of approximately $15 \mathrm{~g}$ of normal food. In previous studles (Struyker-Boudier, 1981) it has been shown that rats reach equilibrium between intake and excretion of water and sodium whin 8 hr after the start of the infusion. Fourty-eight hr before and during the experiments normal food was replaced by sodium-depleted food (see above). During the experiment, rats were housed in metabolism cages (see above). The outlet of the cages was connected to LKB model 7000 Ultrorac fraction collector, which was set to change (preweighed) tubes every 90 min. Connections were made with silicone tubing and a peristaltic pump was included into the system to ascertain constant flow of urine through the tubing. Tubes were reweighed and the volume of the urine calculated from the weight 
increase assuming a specific grawity of $1 \mathrm{~g} / \mathrm{ml}$. Sodium and potassium concentrations were measured in all samples wh an Eppendorf flame photameter using standard methods. Urine osmolarity was determined by freezing-point depression method (Knauer AG Osmometer, Berlin, Germany).

It was found impossible to recover $100 \%$ of the infused water and sodium from the collected urine. Besides from renal loss of water and sodium and loss of water through evaporation in breath, also the fact that large metabolisin cages were used (rats were less disturbed than in small cages) probably contributed to this loss. Nevertheless, sodium recovery was $73 \pm 4 \%$ over a 24 -hr control period (see chapter 6).

\subsection{Measurements of regional blood flows}

Regional blood flows were measured in rats, using a 20-MHz directional pulsed Doppler flowmeter (Bio-engineering Resource Facility, University of Iowa, Iowa City, Ia, USA) w th miniaturized Doppler probes. Following an upper mid-abdominal incision (pentobarbital anesthesia), Doppler flowprobes were placed, depending on the experimental protocol, on one or both renal arteries $(0.8-0.9 \mathrm{~mm})$, the supertor mesenteric artery $(0.9 \mathrm{~mm})$ and abdominal aorta $(1.2 \mathrm{~mm})$ distal to the iliolumbar arteries (see fig. 2.1). The latter flow consists mainly of blood flow through the skeletal muscle of the hindquarters and will be referred to as hindquarter flow. The abdomen was closed and the probe cables were guided subcutaneously to the neck and soldered to a miniature connector which was mounted on the animal's skull with jeveler's screws and dental cement. Catheters for intrarenal and in tramesenteric infusion were implanted during the same surgical session when necessary. Animals were allowed to recover for at least 3-4 days before intra-arteriml and venous catheters vere inserted.

Reglonal blood $\mathbb{E l o w}$ was measured as $\mathrm{kHz}$ Doppler shift, which is $\mathrm{di}$ rectly and linearly proportional to volume flow (Haywood et al., 1981). Zero blood flow was determined electronically. Mean flow was obtained and recorded by low-pass filtering of the pulsatile signal on a Grass 70 poly graph. Organ vascular resistance (renal resistance: RR, mesenteric resistance: MR, hindquarter resistance: $H Q R$ ) was calculated as the quotient of pressure and organ blood flow. 


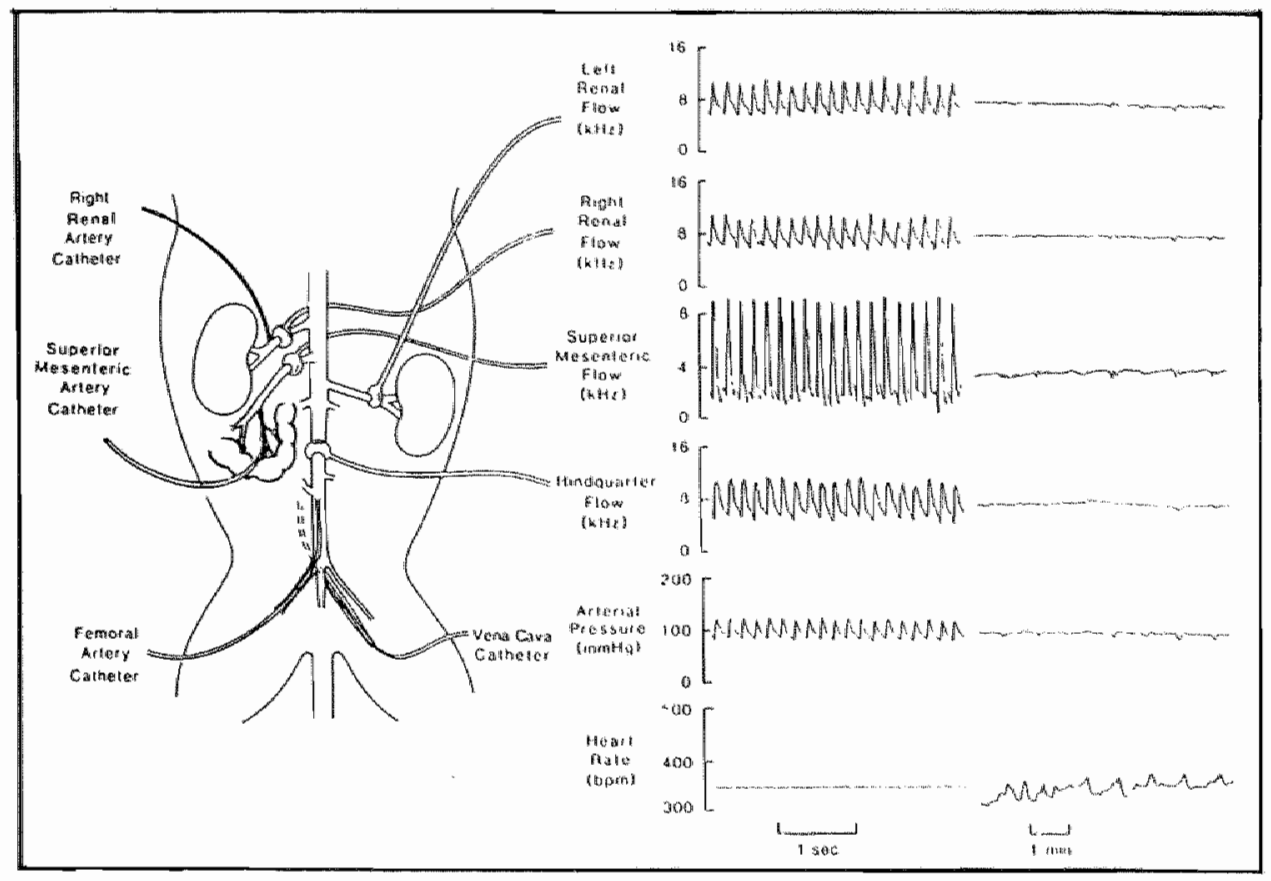

Fig. 2.1 Experimental lay out of the animal preparation for chronic iv, ir and im infusion techniques, blood pressure measurements and regional blood flow recordings.

\subsection{Electrophysiological recordings of afferent renal nerve acitivity}

Measurement of afferent renal nerve acitiwity in rats has been described in detall by amongst others Recordati et al. (1978) and Moss et al. $(1985)$. Briefly, rats $(250-300 \mathrm{~g})$ were fasted overnight, anesthet 1 ed with sodium pentobarbital and body temperature maintained at $37^{\circ} \mathrm{C}$ on a heated operating table. The trachea was intubated 1 th a PE-240 cannula to faci1itate breathing. Two PE-10 catheters were inserted into the left external jugular vein, for continuous infusion of $0.9 \% \mathrm{MaCl}(40 \mathrm{\mu l} / \mathrm{min})$, and supplemental pentobarbital injections for maintaining anesthesia. The left femo-. ral artery was cannulated with PE-50 to monitor arteriall blood pressure. A PE-50 catheter was inserted to drain the bladder.

Each rat was placed on its left side. The right kidney and its nerve supply were exposed retroperitoneally through a paravertebral incision. The 


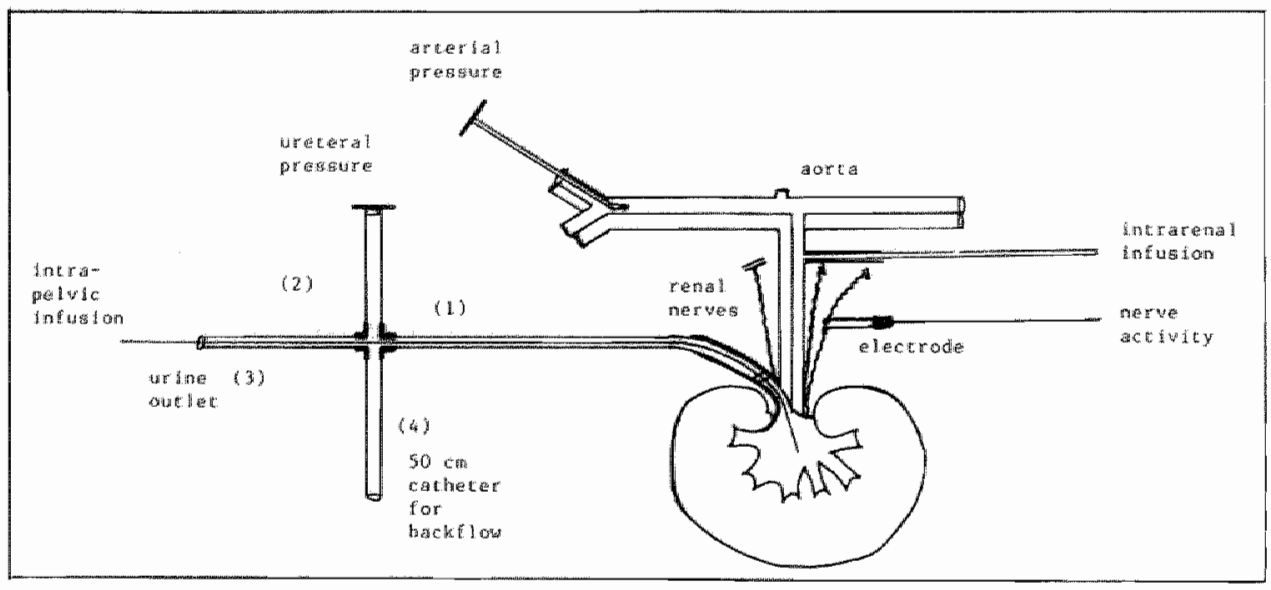

Fig. 2.2 Experimental lay-out of the animal preparation for recording of afferent renal merve activity. For details, see section 2.8 .

right ureter was cannulated with PE-50 tubing, which allows urine to flow freely and connected to a 4-way construction of PE-50 catheters as shown in fig. 2.2. Via branch 3 (urine outlet) and branch 1 , a tapered PE-10 cannule was inserted into the renal pelvis for intrapelvic administration of drugs. Under clamping of branch 3 , backflow of urine into the renal pelvis could be accomplished by ralsing branch $4(50 \mathrm{~cm}$ long), while ureteral pressure was continuously monitored through branch 2 . For backflow of test solutions into the pelvis, branch 4 was filled while clamping branches 1 and 2 . The suprarenal artery was cannulated with tapered PE-10 for direct application of drugs into the renal blood stream.

Under mineral ofl. the renal. nerves were dissected from the surrounding tissues on each side of the renal artery, from the hilus to their function with the coeliac ganglion. They were then cut centrally and positioned on platinum bipolar hook electrodes. All connections to the greater splanchic and all ather visible nerves joining the coeliac plexus were severed, to $\$$ solate the kidney from efferent neural influences. The electrical signal was premamplified with a differential amplifier, passed through a band-pass filter with frequency cut-offs set at $100 \mathrm{~Hz}$ and 1000 $\mathrm{Hz}$ and displayed on an oscilloscope. Electrical nerve impulses exceeding the background noise level. were counted by nerve activity monitors and displayed as counts/s on a Grass 70 polygraph. Also ureteral pressure, heart rate, and arterial blood pressure were recorded continuously. Simultaneous- 
1y, all parameters were stored on magnetic tape for later computer analysis.

\subsection{Plasma and tissue analyses}

\subsubsection{Determination of plasma renin concentration}

The assay was performed with the aid of $\mathrm{dr}$. F. Derkx in the department of Internall Medicine, Dijkzigt Hospital, Erasmus University, Rotterdam, The Netherlands. Plasma renin concentration (PRC) was determined as the amount of angiotensin I (AI) generated by $1 \mathrm{ml}$ of plasma after $1 \mathrm{hr}$ incubation at $37^{\circ} \mathrm{C}$ in the presence of excess renin substrate (angiotensinogen). Plasma was obtained from blood samples containing $100 \mathrm{mg} / \mathrm{ml}$ Na, EDTA and $11 \mathrm{mg} / \mathrm{ml}$ O-phenantroline.

Fifty $\mathrm{\mu l}$ of plasma and $150 \mu \mathrm{L}$ of renin substrate (prepared from plasma of anephric rats) were incubated for $1 \mathrm{hr}$ at $37^{\circ} \mathrm{C}$ in $100 \mu \mathrm{l}$ phosphate buffer ( $\mathrm{pH}$ 6.0) and $13.5 \mu \mathrm{l}$ of a solution containing inhibitors wich prevent the breakdown of newly formed AI. The phosphate buffer contained 12.2 $\mathrm{mM} \mathrm{NaH} \mathrm{PO}_{4}, 86.7 \mathrm{mM} \mathrm{Na}_{2} \mathrm{HPO}_{4}, 75.9 \mathrm{mM} \mathrm{NaCl}$ and $1.0 \mathrm{mM} \mathrm{Na} 2$ EDTA; pH 6.0). The inhibitor solution contained a mixture of $5 \%(w / v)$ phenyl-methyl sulfonyl fluoride solution, a $10^{9} \mathrm{IU} / \mathrm{ml}$ trasylol solution, a $100 \mathrm{mmol} / \mathrm{I} \mathrm{Na}_{2} \mathrm{EDTA}$ solution, a $10 \%(w / v)$ neomycin sulphate solution and $0.34 \mathrm{~mol} / 1$ 8-oH quinoline sulphate solution in a $1: 2: 2: 2: 2$ ( $v: v: v: v: v)$ ratio. One $50 \mu 1$ ( $t: 0)$ and three $50 \mu 1 \quad(t=60)$ aliquots were used for the radloimmunoassay to determine AI. These 50- 1 l samples were mixed with $250 \mu 1$ tris/acetate buffer (0.1 M Tris, $0.35 \%(w / v)$ bovine serum albumin, $0.15(w / v)$ lysozyme and $0.2 \%$ ( $w / v)$ neomycin sulphate adjusted to $\mathrm{pH} 7.5$ with glacial acetic acid, $50 \mu l$ ${ }^{125}$ I - AI solution (approximately $7000 \mathrm{cpm}$ ) and 250 ul ant $1-11 \mathrm{e}^{5}$ AI rabblt antiserum and incubated overnight at $0^{\circ} \mathrm{C}$.

Then, $500 \mu l$ of a charcoal suspenston was added to the incubate to separate the antibody-bound AI. The charcoal suspension consisted of $2.5 \mathrm{~g}$ Dextran $\mathrm{T} 70$ (Pharmacia) and $25 \mathrm{~g}$ of charcoal (Sigma) in $1 \mathrm{l}$ barbital buffer (131 mM NaCl, $7.1 \mathrm{mM}$ sodium barbital and $7.1 \mathrm{mM}$ sodium acetate; pH 7.5). After mixing and centrifugation $\left(10 \mathrm{~min}, 2000\right.$ rmp at $\left.20^{\circ} \mathrm{C}\right)$, the charcoal precipitate and the supernate were counted separately in a gamma-counter (LKB). PRC is expressed as $\mathrm{ng} \mathrm{AI} / \mathrm{ml}$ plasma per $\mathrm{hr}$.

\subsubsection{Determination of plasma and $t$ issue norepinephrine concentration}

The assay of NE was performed by high-performance liquid chromato- 
Graphy (HPLC) combined with coulochemic detection (analytical cell: model 5011 Coulochem 5100 A ESA, Bedford, Mass, USA) modified from the method described by Sifiedes et al. (1982). For renal tissue WE determination, kidneys were quickly excised and the renal capsule was removed. After rinsing in ice-cold saline, the kidneys were blotted dry with filter paper and welighed. Then they were immediately transferred to an ice-cold perchloric acid solution $(8 \mathrm{ml}, 0.4 \mathrm{~N}$ containing $800 \mathrm{ng} \mathrm{3,4-dihydroxybenzylamine} \mathrm{as} \mathrm{an}$ internal standard) and homogenized, using an Ultra-Thurrax homogenizer. The homogenate was centrifuged for $10 \mathrm{~min}$ at $3000 \mathrm{~g}$ and the supernate stored at $-80^{\circ} \mathrm{C}$ until assayed by HPLC. For plasma NE determination, a $0.5-m 1$ blood sample was taken wia an extension of the arterial cannule without disturbing the rats. Heparine and glutathion ( $75 \mu \mathrm{g}$ ) were added and after centrifugation plasma was stored at $-80^{\circ} \mathrm{C}$ unt 11 assayed.

Tissue samples were prepared for HPLC by the following procedure: to extract the catecholamines, $50 \mathrm{mg}$ activated $\mathrm{Al}_{2} \mathrm{O}_{3}$ and $25 \mathrm{mg} \mathrm{Na}_{2}$ EDTA were mixed with $5 \mathrm{ml}$ of the homogenate, adjusted to $\mathrm{pH} 8.8$ and shaken vigorously for 10 min. After the $\mathbb{1}_{2} \mathrm{O}_{3}$ was washed 4 times with ultrapure water, the catecholamines were eluted by adding $1 \mathrm{mI} \quad 0.05 \mathrm{~N} \mathrm{HClO}_{4}$ to the $\mathrm{Al}_{2} \mathrm{O}_{3}$ and shaken for 5 min. After centrifugation ( $1 \mathrm{~min}$ at $1000 \mathrm{~g}$ ) $100 \mu 1$ of the supernate was injected into the HPLC system (see below).

Plasma samples were prepared for HPLC by the following extraction procedure. Five hundred $\mu \mathrm{I}$ buffer and $100 \mu \mathrm{l}$ of an aqueous solution containing dihydroxybenzylamine ( $2 \mathrm{ng} / \mathrm{ml}$; internal standard), were mixed with 100 Hl plasma. The $0.5 \mathrm{M} \mathrm{NH}_{4} \mathrm{OH}-\mathrm{NH}_{4} \mathrm{Cl}$ buffer (pH 8.6) contalning $0.03 \%$ $(w / v)$ diphenylborate-ethanolamine and $0.015 \%$ ( $w / v$ ) $\mathrm{Na}_{2}$ EDTA had been washed by activated $\mathrm{Al}_{2} \mathrm{O}_{3}$. After addition of $1.8 \mathrm{ml} \mathrm{N}$-heptane containing $1 \%(\mathrm{v} / \mathrm{v}$ ) $\mathrm{n}$-octanol and $0.09 \%(\mathrm{v} / \mathrm{v})$ tetra-octylammoniumbromide, the sample was shaken wgorously for $5 \mathrm{~min}$ and centrifuged at $1000 \mathrm{~g}$ for $10 \mathrm{~min}$. Next the upper organic phase was transferred to a tube containing $750 \mu \mathrm{h} \mathrm{n-octanol}$ and 150 fil $0.08 \mathrm{M}$ acetic acid to which $0.02 \%$ EDTA was added. After the tube was shaken for $3 \mathrm{~min}$ and centrifuged for $5 \mathrm{~min}(1000 \mathrm{~g}), 150 \mathrm{Hl}$ of the aqueous phase were injected into the HPLC system. The analytical column was a chrom Spher C12 column (10 cm $\times 3 \mathrm{~mm}$ ID; Chrompack Int., Middelburg, The Netherlands) connected in series with a Supelco $\mathrm{C18}$ column (15 cm $\times 4.6 \mathrm{~mm}$ ID; Supelchent, Leusden. The Netherlands). The eluent was composed of ultrapure water, $1.2 \%$ (w/v) HAc $0.17 \%$ (w/v) $\mathrm{NaOH} 0.02 \%$ (w/v) $\mathrm{Na}_{2}$ EDTA and $0.035 \%$ (w/v) $\mathrm{C}_{8} \mathrm{H}_{17} \mathrm{NaSO}_{3}$ adjusted to $\mathrm{pH} 4.06$. Then, $0.3 \%(\mathrm{v} / \mathrm{w})$ ACCN was added. Before use, the solution was filtered and degassed by sonification at low pressure. The intra- and interassay coefficients of variation were $9 \%(n=14)$ 
and $12 \%(\mathrm{n}=15)$, respectively.

\subsection{Data processing and statistical analysis}

In most of the experiments, hemodynamic data were digitized and stored on disc by a mini-computer (MINC RT-11) or personal computer (Apple IIe or 0livetti M28). Data were sampled "on line" (every second) and the mean values over longer periods $(5,10$ or $15 \mathrm{~s}$, depending on the experimental protocol) were stored for analysis. Absolute values and absolute and percentage changes of hemodynamic parameters were calculated ith computer ass is tance *

The following statistical methods have been used to compare hemodynamic data in several experimental groups:

1. Baseline hemadynamic values before the start of an experiment between 2 groups were compared with a Student's t-test for unpained observations. If more than 2 groups were compared, a one-way analysis of variance followed by a modified t-test (Wallenstein, 1980) was applied.

2. Multiple comparisons of hemodynamic values between 2 or more groups and evaluation of changes over time were subjected to analysis of variance for repeated measurements, whereafter appropriate procedures were applied (Wallenstein, 1980).

3. Comparison of blood pressure development curves beveen 2 groups (chapter 9) were analyzed by a one-way analysis of variance for growth curves (Zerbe, 1979) using a VAX computer.

All data are presented as means \pm SEM, unless indicated othervise. Significance was accepted at the $95 \%$ confidence level. 
3. ELECTROPHYSIOLOGICAL CHARACTERISTICS OE CHEHOSENSITIVE APFERENT RENAL NERVES

\subsection{Introduction}

The afferent renal nerves (ARN) have been studied with electrophysiological techniques for more than 25 years in cats (Pines, 1960; Aström and crafoord, 1968; Beacham and Kunze, 1969), dogs (Uchida et al., 1971; Katho11 et al., 1983), rabbits (Nijjima, 1971, 1972, 1975), rats (Astróm and Crafoord, 1967; Recordati et al., 1978, 1980; Barber et al., 1985, 1986) and non-humari primates (Gilmore and Tomomatsu, 1985). Yet the physiological or pathophyslological conditions under which they alter their discharge rate are still unclear. Afferent renal nerve activity can be induced by the action of mechanlical or chemical stimuli on the receptive nerve endings within the kidney. Mechanoreceptors have been described in several species. this receptor population is sensitive to increases in renal arterial pressure, renal vein pressure, ureteral pressure or compression of the kidney (Moss, 1982, 1985, 1987). They appear to be located in the renal medulla and pelvis (Knuepfer and Schram, 1985). In most of the studies the pressor stimuli used to activate the mechanoreceptors were of ten beyond the bounds of normal range. Nevertheless, there is general agreement that increased renal venous pressure is an adequate stimulus for these mechanoreceptors.

Renal chemoreceptors have been studied in most detail in the rat, where two types, designated $R_{1}$ and $R_{2}$ chemoreceptors, have been described (Recordat i et al., 1978, 1980). $\mathrm{R}_{1}$ chemoreceptors have no basal discharge, are unresponsive to changes in renal arterial, venous, or intrapelvic pressure, but are activated when renal blood flow is stopped or reduced to very low levels by renal artery occlusions. It should be noted that $\mathrm{R}_{1}$ chemoreceptors do not become immediately active after applying the ischemic stimu11; about $30-40 \mathrm{~s}$ elapse until a discharge appears in the form of trains of impulses. The activation persists for about $90 \mathrm{~s}$ and then disappears, de-spite continued renal ischemia. The nature of the stimulus for these receptors is stild unclear. The observations suggest that an accumulation of a chemical substance which is released during renal ischemia is needed to activate these nerves. Whatever the stimulus, their resistance to stimulation except under extreme circumstances, excludes these receptors from a normal physiological role. 
$R_{2}$ chemoreceptors exhibit a basal discharge and are activated by backflow of hypertonic urine or sodium solutions $(500-950 \mathrm{mH})$ or potassium solutions (50-100 $\mathrm{mM}$ ) into the renal pelvis. Their spontaneous activity can be suppressed by backflow of normal saline or diuretic urine (Recordati, 1978). Recent ewidence suggests that moderate stepwise reductions in renal. perfusion pressure increase $R_{2}$ chemoreceptor discharge (Barber and Moss, 1987). In contrast to $\mathbb{R}_{1}$ chemoreceptors they show a prompt excitation upon renal artery occlusion that persists for $20 \mathrm{~min}$ or more. These observations indicate that these $R_{2}$ chemoreceptors might be ideally located to monitor the chemical composition of their environment, and in this role they may contribute to physiological or pathophysiological mechanisms in body fluid homeostasis.

Evidence has recently become available that ARN may play an important role in hypertension (Katholi, 1983, 1985; Janssen and Smits, 1988). Several chemoreceptor stimulants such as bradykinin (BK) and adenosine (ADO) have been infused directly into the renal bloodstream to study the hemodymamic reflex effects which have been shown in renal denervation studies to be ARN dependent. These investigations are, however, only poorly supported by evidence from electrophysiological studies. Intrarenal infusion of $B K$ (Smits and Brody, 1984) and ADO (Katholi et al., 1983, 1987) elicited apparent ARN depending sympathetic reflex effects in conscious rats or dogs. Yet, only in one study in dogs (Katholi, 1983) a neurogram has been presented to demonstrate that ARN discharge was increased upon ADO application to the kidney. Electrophysiological recordings during intrarenal BK and ADO infusions in the rat have not been performed yet.

The aim of the present investigations was to study the characteristics of rat renal chemoreceptors. In more detail, ARN responses were examined during intrarenal and intrapelvic infusion of BK, $A D O$ and norepinephrine (ME) to provide an electrophysiological basis to interpret the cardilovascular changes, which occur when these drugs are infused into the renal circulation of conscious rats.

\subsection{Experimental protocol}

Sprague Dawley rats $(250-300$ g) were prepared for afferent renal nerve recordings as described in section 2.8 . Once a small multifiber unit preparation had been isolated with a stable spontaneous electrical activity showing spike amplitudes exceeding at least 3 times the level of background 
noise, experiments were started. In each experiment baseline afferent renal nerve activity (ARNA) was recorded for several minutes. Then, drugs were infused ither directly into the renal blood stream (ir) or into the renal pelvis (1pv) to test their action on ARNA (see fig. 2.2). The next experiment was started when ARNA had returned to baseline levels of spontaneous activity for 10 minutes. The following substances vere tested:

1. Sallne: Since all drugs were dissolved in $0.9 \% \mathrm{NaCl}$, measurements of ARNA were made at several infusion rates of the wehicle solution. Saline was Infused $1 \mathrm{r}$ and $1 \mathrm{pv}$ at $20 \mu \mathrm{I} / \mathrm{min}$ and this rate was increased stepwise by $20 \mu 1 / m i n$, ewery two minutes until a maximum of $100 \mu 1 / m i n$ was reached.

2. Adenosine: ARMA was recorded during ir or ipv infusion of ADo ( $1 \mu g / \mu 1)$ at an initial rate of $20 \mu 1 / \mathrm{min}$. The infusion rate was increased every two minutes by $20 \mathrm{\mu L} / \mathrm{min}$ until a maximum of $100 \mathrm{\mu L} / \mathrm{m}_{\mathrm{in}}$ was reached.

3. Norepinephrine: To study whether NE could result in an increase of ARNA as suggested by Kleinjans et al. (1984), comparable doses $(20-200 \mathrm{ng} / \mathrm{kg}$. hr) as used in those studies were infused intrarenally for 5 to 10 minutes in random order while recording ARNA. In several experiments a different protocol was followed and NE was applied in an increasing dose up from 20 to $200 \mathrm{ng} / \mathrm{min}$.

4. Bradykinin: A BK solution of $50 \mu \mathrm{g} / \mathrm{ml}$ of the tri-acetate salt was infused either ir or ipv for 5-15 minutes in random order at a rate of 6 , 20 or $60 \mu 1 / m i n$. This rate was chosen to deliver equal amounts (respectively $0.3,1.0$ or $3.0 \mathrm{ug} / \mathrm{min}$ ) of the drug into the renal circulation or pelvis as applied in the experiments in which the BK induced ARN dependent reflexes in consctous rats were studied (chapters 4 and 5).

5. Backflow of urine: To compare the drug induced changes in ARNA with a "standard" stimulus, urine was refluxed before and/or at the end of the Infusion period. In a few experiments this manoeuver was also done du$r i n g$ the drug infusions. Since backflow of urine only excites $\mathbb{R}_{2}$ chemoreceptors, this procedure could reveal information about the subpopulatons of renal receptors which are stimulated by the drug. Furthermore, results frotil pilot studies on the effects of furosemide on ARNA will be presented. These studies were performed in addition to the experiments described in chapter 7 , in which the acute hemodynamic effects of furosemide were examined.

6. Data presentation and analysis: Results are presented in direct chart recordings or in computer-aided graphics to obtain a better resolution in ARNA. Mean changes \pm SE in ARNA (percentage from control) are presen- 
ted for the intrarenal infusion experiments with BK, ADO and NE. Furthermore, original figures are presented of several pilot experiments, which are 11 lustrative for the characteristics of the ARM.

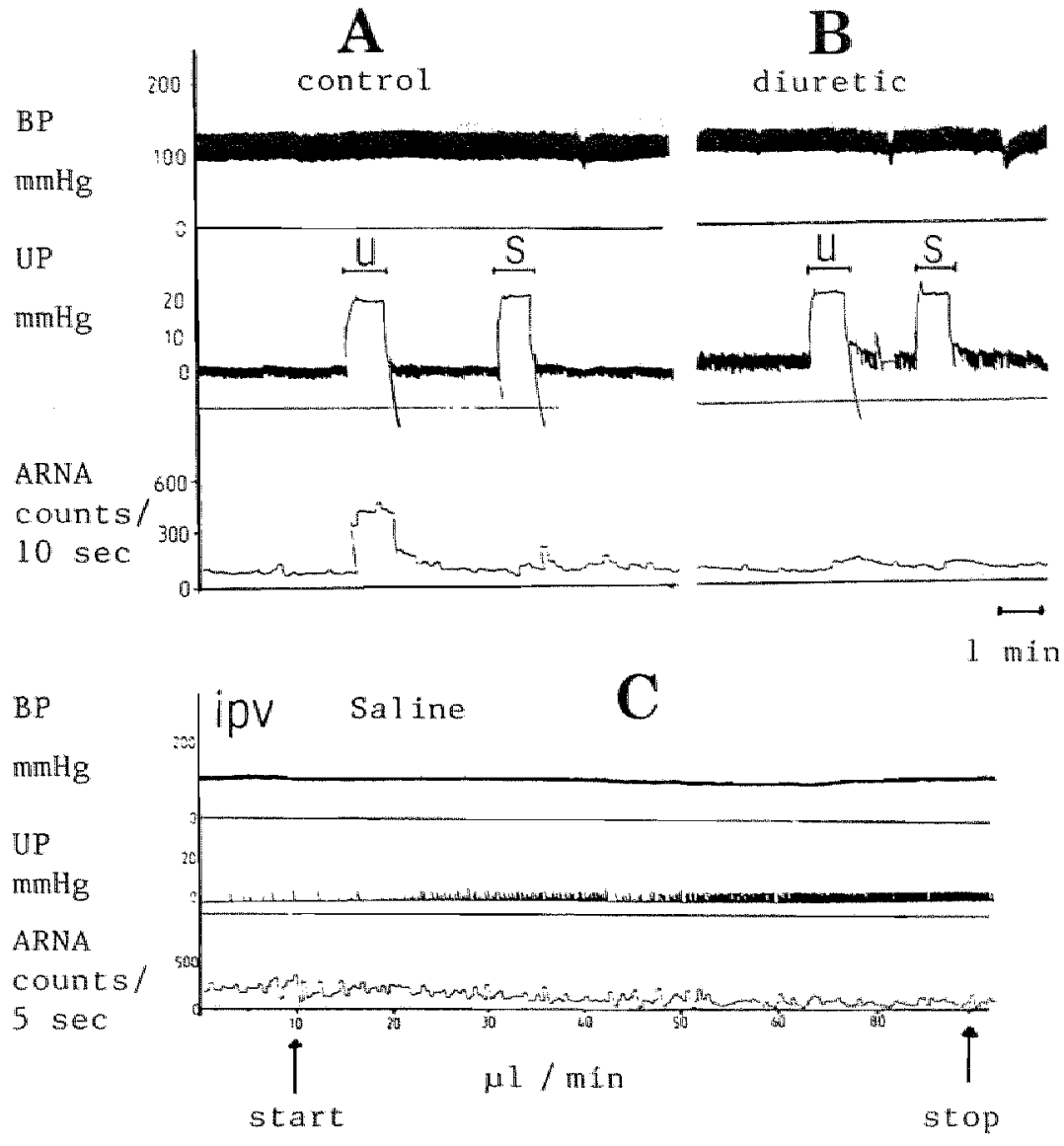

Fig. 3.1 Multi-unit ARN responses to backflow of urine (U) and isotonic saline (S) in a control rat (A) and in a rat pretreated with furosemide (B). Multi-unit ARN responses to stepwise increasing ipw perfusion with isotonic saline (C). 

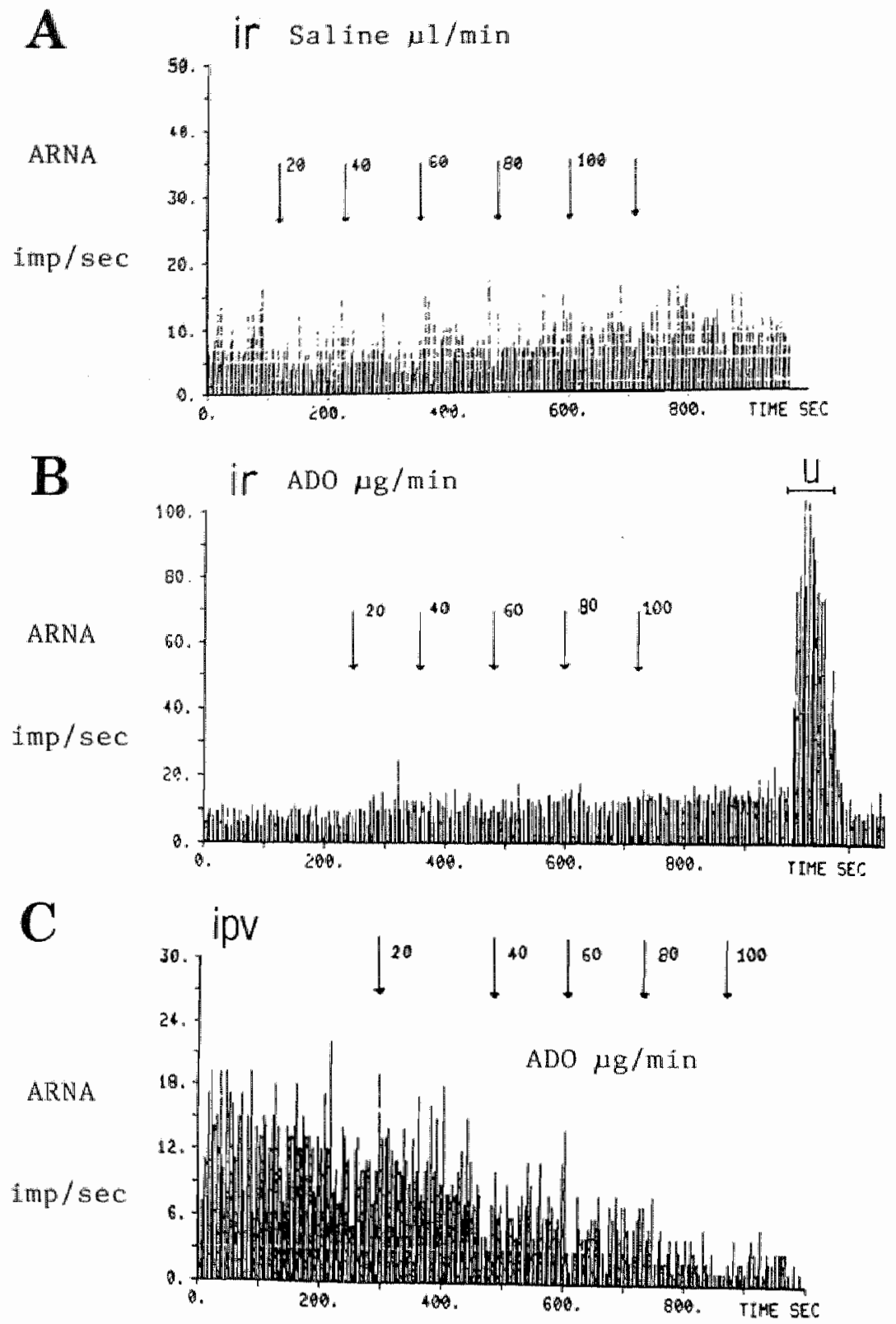

Fig. 3.2 Mult1-unit ARNA during stepwise increasing ir infusions of saline (A) and adenosine (B). Multi-unit ARN responses to pelvic perfusion wh adenosine (C). 


\section{3 Results}

In 8 rats extracellular multi-unit afferent renal nerve recordings were performed. All multifiber preparations exhibited spontaneous activity. Mean baseline discharge was $7.4 \pm 1.0$ impulses/s (range $3-12$ itmp/sec), indicating that in these nerve preparations a comparable number of $R_{2}$ chemoreceptors were active.

Characteristic multi-unit ARNA to backflow of urine and isotonic saline into the renal pelvis of a non-diuretic rat are shown in fig. 3.1A. Typically ARN responded to backflow of urine but were not sensitive to backflow of isotonic saline (145 $\mathrm{mM} \mathrm{NaCl}$ ) at similar intrapelvic pressures of $20 \mathrm{mmHg}$. This indicates that the response is due to chemoreceptor activation rather than mechanoreceptor stimulation. Hean activation of ARN was $368 \pm 61 \%(n=6)$ over control levels during backflow of urine and $40 \pm 11 \%$ $(n=6)$ during backflow of isotonic saline at the same (20 mmHg) intrapelvic pressure. In a diuretic rat wich was given furosemide ( $8 \mathrm{mg} / \mathrm{kg}$ iv) ARN discharge to backflow was clearly impaired (fig. 3.1B).

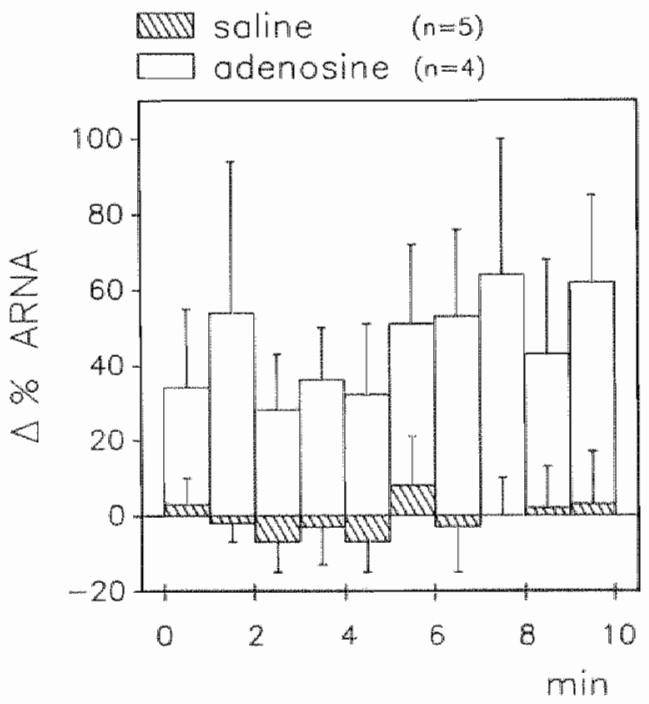

Fig. 3.3 Average increases in ARNA during stepwise increasing infusions of saline and adenosine $(10 \mu \mathrm{g} / \mu \mathrm{l})$. Starting at an inftial rate of 20 $\mu l / m i n$, the infusion rate was ralsed every 2 min by $20 \mu \mathbb{m}$ in. 


\subsubsection{Responses to intrarenal infusion and pelvic perfusions}

Saline: Typical mult-unt ARNA recordings for ir infusion of saline are presented in fig. $3.2 \mathrm{~A}$. Intrarenal infusion of saline from $20-100 \mu 1 /$ mim hadl no influence on multi-unit ARNA. Mean values are shown in fig. 3.3 . In the experiments $(n=2)$ in which the renal pelvis was perfused with saline basal ARMA decreased progressively w the increasing infusion rates (see fig $3 \cdot 1 \mathrm{C})$.

Adenosine: Direct infusion of $\mathrm{ADO}$ into the renal blood stream increased ARNA slightly but significantly (ANOVA p<0.01) above controll levels. A typical example is given in fig. 3.2B (mean values are shown in fig. 3.3). No clear dose related response in ARMA was present, which is probably due to the fact that relatively high infusion rates were used. The effects of the highest infusion rate $(100 \mathrm{\mu g} / \mathrm{min})$ resulted only in a minor activation of ARN, as compared to the effects evoked by backflow of urine (cf. fig. 3.2B) or ir infusion of BK and NE. Mean arterlal blood pressure fell during the $A D O$ infusions with a maximum decrease of $20 \mathrm{~mm}$ at at 100 $\mu g / m i n$. Pelvic perfusion with ADO (2 experiments) did not affect arterial blood pressure and had also no excitatory effect on ARNA. At increasing infusion rates, basal ARNA even declined progressively in a comparable way as during lpv saline infusions (see fig. 3.2C).

Norepinephrine: Fig. 3.4 illustrates the typical ARN response to increasing doses of ir applied NE. ARNA increased from $20 \%$ at $20 \mathrm{ng} / \mathrm{min}$ to even 400-500\% at a maximum rate of $200 \mathrm{ng} / \mathrm{min}$. Usually there was a time delay of approximately $1.5 \mathrm{~min}$ before responses could be observed ". ARNA was highest when the kidney, due to the NE induced wasoconstriction turned. completely white. Urine flow stopped at that moment. The dose at which this

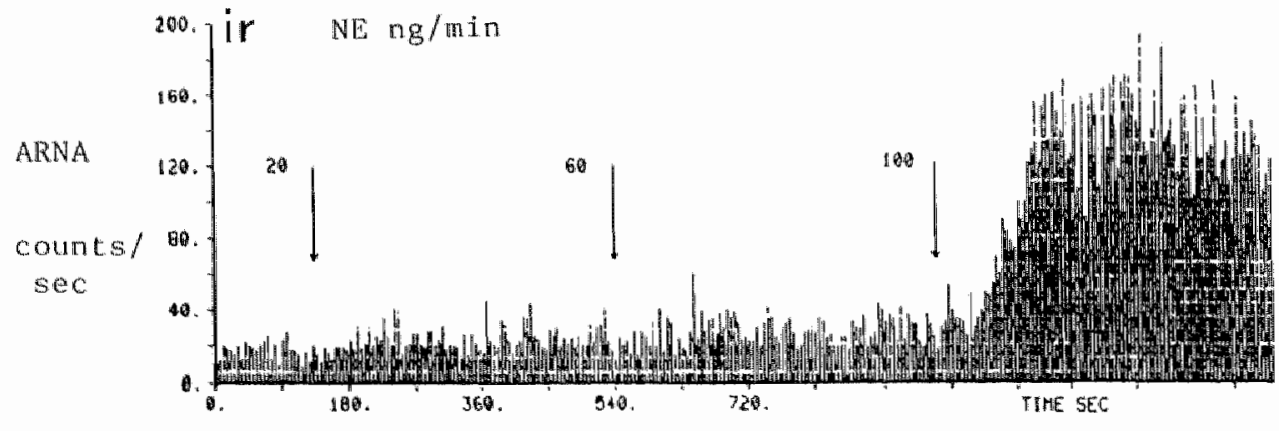

Fig. 3.4 Mult 1 -unit ARM response to increasing doses of ir infused NE. 
occurred was wariable. In some preparations maximal ischemic response was already visible at $60 \mathrm{ng} \mathrm{NB} / \mathrm{min}$ wile in others $1 \mathrm{t}$ occurred only at 200 ng/min. Further increment of the NE infusion rate was then withowt further effect on ARNA. Interestingly, during the prolongation of a maximal effective dose of $\mathbb{N E}$, the kidney escaped visually from ischemia for short periods, while no clear changes in ARNA occurred. In 11 . 3.5 the mean ARN responses to several dases of it infused NE are shown. Blood pressure was maximally increased with $20 \mathrm{mmlg}$ at the highest infusion rate (200 $\mathrm{ng} / \mathrm{min})$. In a single experiment $\mathrm{NE}$ was infused at increasing rates $(20-200 \mathrm{ng} / \mathrm{min}$ ) into the renal pelvis. No changes in ARNA could be detected (recording not shown).

Bradykinin: Intrarenal infusion of $B K$ elicited a dose dependent ( $t$ me delay 5-10 sec) increase in ARNA, which was highest during the first minute of infusion and declined gradually to somewhat lower levels when the infusion was prolonged (mean values are presented in fig. 3.6). High infusion rates $(3.0 \mathrm{\mu g} / \mathrm{min}$ ) evoked a maximal ARNA response comparable to that occurcing during the in NE induced ischemic response, while no changes in blood pressure or ureteral pressure occurred. In about half of the $B K$ experiments a typical ARNA pattern as shown in fig. 3.7 was present. This BK

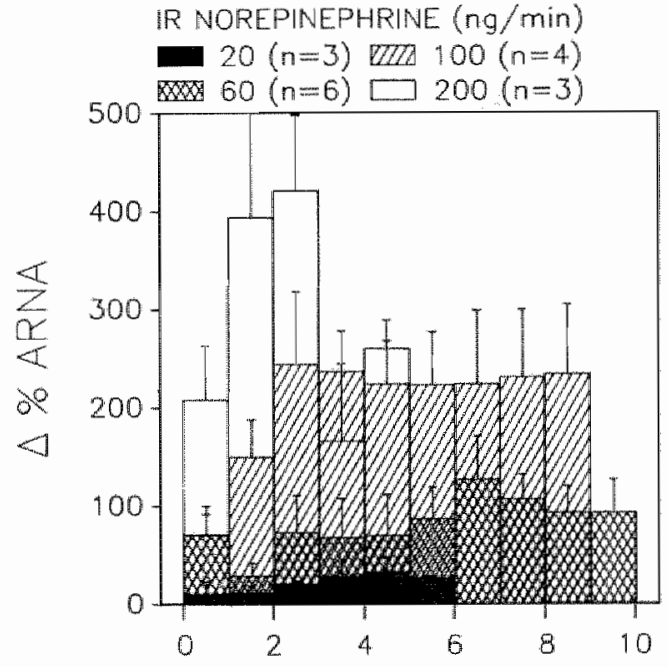

Fig. 3.5 Mean ARN responses to several doses of ir infused NE.

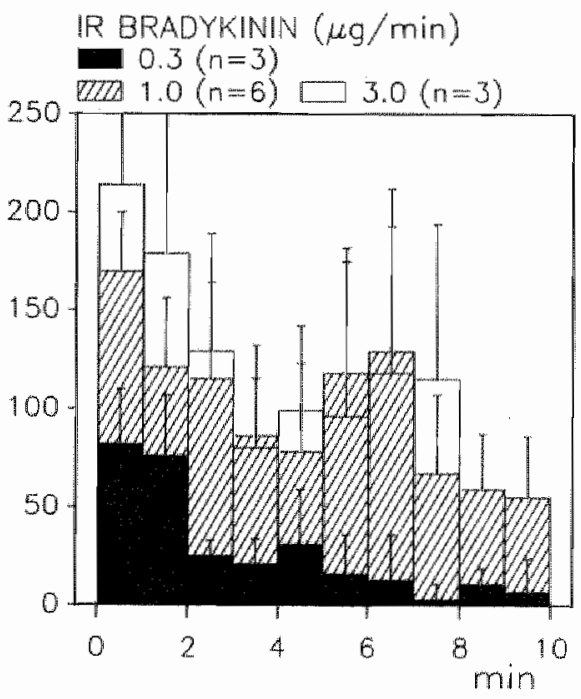

Fig. 3.6 Mean ARN responses to several doses of ix infused $B K$. 
induced thy thic activity was not dose dependent and could be eliclted by It as well as Ipv BK Infuston (see fig. 3.7C). Rhythmicity became, however, less apparent when the infusion was prolonged for longer times. At high ipv infusion of the BK solution the stimulatory effect of BR on ARNA was less pronounced and ultimately fell to baseline discharge values at 100 ul/min.
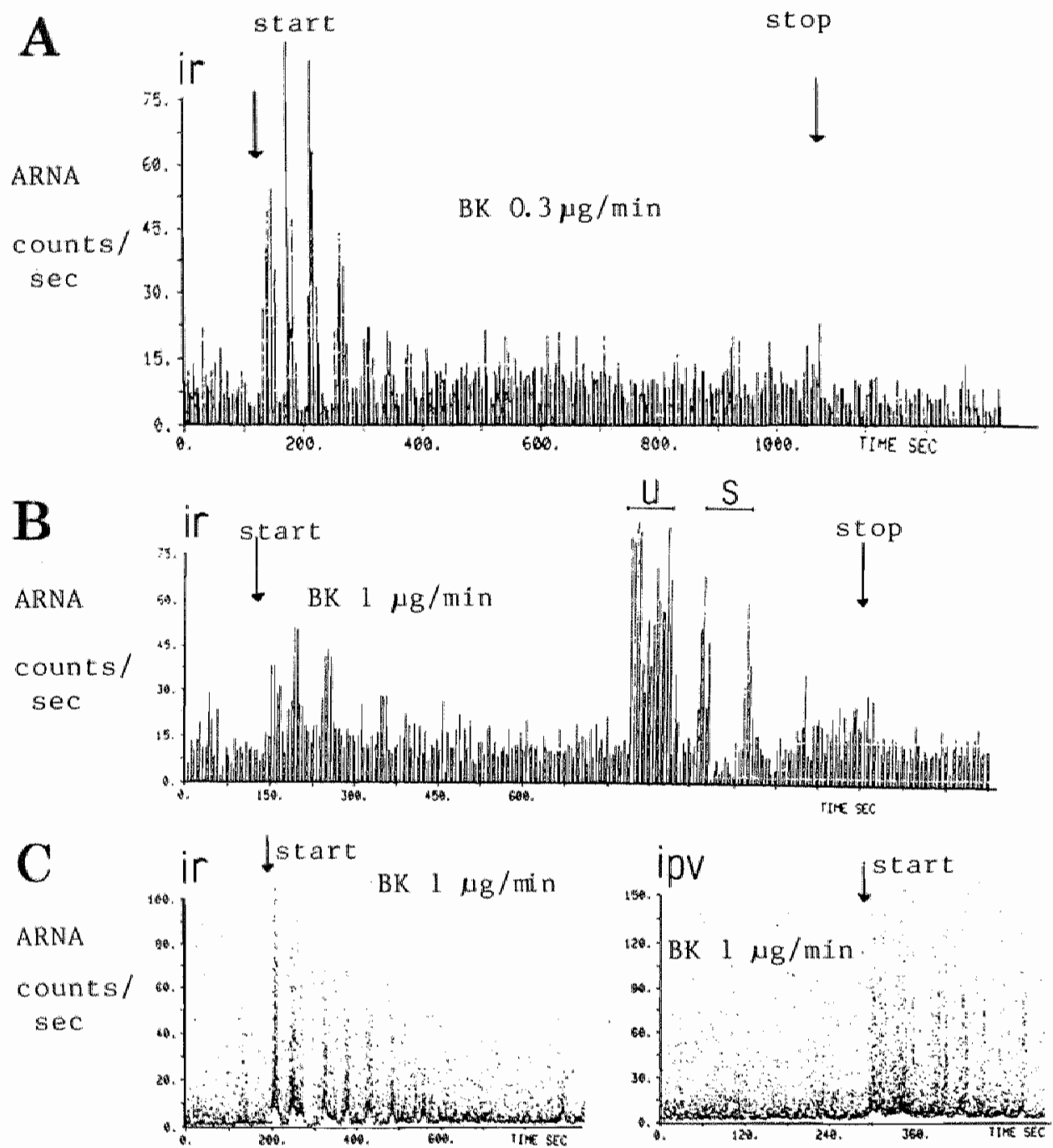

Fig. 3.7 A, B and C: Multi-unt ARN responses to it infusion of BK. B: ARN response to backflow of presampled urine (U) and isotonic saline (S) during ir BK infusion. C: ARNA/Dt during ir as well as ipv BK infusion. 
This reduction in ARNA is probably due to the same "wash-out" effects as observed by ipv saline and ADo infusions. Similar observations vere made when during ir $B K$ infusion, saline was refluxed into the pelvis (tIg * 3.7B). Backflow of pre-sampled non diuretic urine increased ARNA markedly.

In two experiments the effects of furosemide $(8 \mathrm{mg} / \mathrm{kg} \mathrm{iv})$ on ARNA were investigated. Urine flow increased from $10 \mathrm{\mu l} / \mathrm{min}$ to maximally 120 HI/min. ARNA did, hovever, not change or, if anything, increased slightly. Although the responses to backflow of urine were reduced as compared to the results obtained in a non-diuretic animal ( $\mathrm{flg} .3 .1 \mathrm{~B}$ ), the responses to ir BK infusion were not influenced by furosemide.

\subsection{Discussion}

The present study demonstrates that in the rat application of BK, ADO and NE into the renal bloodstream elicits an activation of ARN. Since ARNA recordings were obtained from multifiber preparations no flrm conclusion can be drawn about the exact nature of the receptors stimulated here. In fact, when these drugs are applied, some ARN populations may cease firing, while others become excited. Furthermore 1 should be recognized that besides chemoreceptors also mechanoreceptors might be inwolved. Since in the present studies on $B K$ and $A D O$, arterial pressure, was slightly reduced and ureteral pressure unchanged, changes in ARNA are probably not due to renal mechanoreceptor stimulation. Whether renal mechanoreceptors become activated during the ir NE infusions is unknown. Characteristically renal mechanoreceptors react and adapt fast (Knuepfer and Schramm, 1985; Uchida, 1971) whereas chemoreceptors maintain their activity when an adequate stimulus is prolonged. Yet, the present recordings show a delayed increment of ARNA after application of NE. Moreover, they resemble the characteristic ARNA response pattern induced by renal artery occlusion or cenal veln occlusion, which result from chemoreceptor rather than mechanoreceptor activation (Recordati, 1978). Taking these considerations together, we suggest that the effects on ARNA during the present infusion experiments wth BK, $A D O$ and NE are mainly due to activation of renal chemoreceptors. Yet, only single unit recondings can give definite answers.

From the fact that maximal intrarenal vehicle $(0.9 \% \mathrm{NaCl})$ infusions, which were about $2 \%$ of renal blood flow, did not influence basal ARNA, it can be concluded that the drug induced changes in ARNA are not disturbed by unwanted wash-out effects. These occur when larger volumes $(2 \mathrm{ml} / \mathrm{min})$ af a 
phisysological salt solution are applied (Recordati, 1978). In contrast, basal ARA fell when the renal pelwis was perfused with saline. Since chemoreceptor endings are probably located near or in the submucosal layers of the pelvis (Notley, 1968; Gosling, 1970; Katholi, 1983), the present data suggest that pelvic perfusion with saline reduces the spontaneous activity af the chemoreceptors by diluting the chemical composition of their receptive fleld. Support for this theory is provided by the observations that ARNA tell also during the high ipo infusion rates of ADO and BR. Furthermore, when saline was refluxed into the pelvis during ir infusion of these drugs a imllar inhibition occurred. This suggests that these chemoreceptor stimulants are only active when the chemical environment of the sensitive receptor (for instance $\mathrm{Na}^{+} / \mathrm{K}^{*} /$ urea concentrations) is not diluted. Thus it may be inferred that $B K$ and $A D O$ stimulate renal chemoreceptors only when they are already spontaneously active. This implies that the conditions for increasing ARNA are also fulfilled. Similar suggestions have been made by Recordatt et al. (1978) on the basis of ARN responses to cyanide injections. ARN were sensitive to this drug only when they exhibited spontaneous activity.

Thus, chemoreceptor stimulants should be dissolved in vehicle solutions of which the composition does not alter basal ARNA when refluxed into the renal pelvis. At the same time this would give more insight in the composition of the chemical environment which prompt basal ARNA.

In the ir infusion experiments ADO elicited a significant but small increase in basal ARNA. In dogs, however, ir infusion of a comparable dose (22 $\mu g / m i n)$ ADO increased multi-unit ARNA by 400\% (Katholi, 1983) which is practically the same as was observed in rats during complete ischemia or maximal BK responses. Many factors such as differences in species, nerve preparation or anesthesia might explain these discrepancies. Yet, the present small response may indicate the existence of a subpopulation of ARN in the rat sensitive to ADO. Furthermore it should be noted that chemoreceptor stimulation during it ADO infusion could also be caused by reductions in renal perfusion pressure or by other substances than ADO itself. Since ADO is known to interact with the renal vasculature, secondary factors released as all result from that interaction may have stimulated the afferent renal nerves, or increased the sensitivity to ADO. More definite statements can be obtained from experinents in which $A D O$ is infused in the presience of substances which inhibit renal vascular reactions or from experiments in which renal blood flow is controlled.

The nona-peptide $B K$ is known as one of the most powerful chemorecep- 
tor stimulants (Regoli and Barabe, 1980). Single-unit recordings from rat renal afferents have revealed that BK speciflcally stimulates $R_{2}$ chemoreceptors with a latency of $8 \mathrm{~s}$, at a threshold concentration of $40 \mathrm{ng}$ wen injected into the renal bloodstream (Moss, personal communication). The present experiments support these observations. Six - 9 s after the start of the BK Infusion ARNA increased enormously in the form of a train of impulses. The effect of BK on ARNA declined in time, which is probably due to tachyphylaxis. In any case baseline ARNA stayed elevated above control levels during the infusion period. Thus, the effects which are described during ir infusion of BK in conscious rats in the following chapters are most likely due to increases in ARN discharge.

A most interesting observation is the BK induced rhythmicity in activation of ARNA. This pattern was not changed by increasing the ir infusion rate and could also be evoked by ipv infusion. Therefore, this rhythmicity does probably reflect an effect of $B(K$ on the sensory nerve endings itself and is not the result of renal vascular interactions. The rhythmicity could reflect an acute tachyphylaxis and hence chemical processes which restore sensitivity to $\mathrm{BK}$ again until the chemical environment is depleted and rhythmicity disappears. We speculate that since $\mathrm{PGE}_{2}$ is a permissive factor in BK induced activation of sensory nerves (ohno et al., 1983; Faber, 1987) the rhythmicity may reflect the local synthesis or release of this substance.

The present experiments provide evidence for the assumption that ir applied NE can increase ARNA (Kleinjans et al., 1984). Already at an ir infusion rate of $20 \mathrm{ng} / \mathrm{min}$ ARNA increased slightly whereas high doses of NE led to renal ischemia and consequently to strong increases in ARNA. Thus, the ARNA response to ir NE is strongly flow dependent. Whether NE itself is a chemoreceptor stimulant is unknown. On the basis of anatomical studies Barajas and Wang (1973) proposed a clase functional relationship between the sympathetic (efferent) and afferent innervation. That hypothesis has been supported by physiological studies from Niljima (1972) and Kopp et al. (1985) who suggested that efferent renal nerve activity plays a tonic permissive role in receptor stimulated ARMA. To get more insight in these processes we need more electrophysiological experiments in which the characteristics of ARMA are examined under several degrees of renal sympathetic stimulation (chemical and electrical) and under controlled renal hemodynamics.

In summary, $\mathrm{ADO}, \mathrm{BK}$ and $\mathrm{NE}$ have been shown to increase multi-unit ARNA when applied to the renal circulation of the rat. The reflus experi- 
ments have indicated that the chemoreceptors are probably located around the renal pelvis and can only be stimulated when they are already spontaneously active. Furthermore, several interesting observations have been made that wartant more investigation. Studies are needed to detect the physiological stimuli for ARM and also the physlological parameters which influence their discharge.

\subsection{Acknowledgement}

The work described in this chapter has been performed during my stay at the Department of Physiology, University of North Carolina at Chapel Hill, NC, USA. I an indebted to dr. Micholas Mass for offering the laboratory faciltties, his experimental assistance and critical reading of the manuscript. 


\subsection{Introduction}

The kidney possesses a considerable amount of mechano- and chemosensitive nerve endings which reflexly influence the cardiovascular system when activated (Moss, 1982). In conscious rats and dogs, stimulation of these ARN Induces pressor responses, mediated by the sympathetic nervous system (Katholi et al., 1983; Smits and Brody, 1984; Faber and Gettes, 1987; Katholi and Woods, 1987). These ARN dependent pressor responses are presumed to contribute to mechanisms initializing several forms of experimental hypertension (Katholi, 1985; Janssen and Smits, 1988).

In parallel to these investigations, recent studies have described mechano- and chemoreceptors in several visceral organs, which also evoke excitatory hemodynamic reflex responses when activated (Tuttle and Mccleary, 1975; 0rdway et al., 1983; 0rdway and Longhurst, 1983; Longhurst et a1., 1984; Lew and Longhurst, 1986; Longhurst and Dittman, 1987; Tobey and Weaver, 1987; Weaver et al., 1987; Meckler and Weaver, 1988; ordway et al., 1988; Stein and Weaver, 1988). However, the functional interpretation of these visceral afferent hemodynamic reflexes is unclear.

The aim of this study is to investigate the specificity and reflex organization of these sensory nerve-evoked renal and visceral reflexes. For this purpose comparisons were made of regional hemodynamic reflex responses elicited by local infusions of chemoreceptor stimulants into the renal. artery and into the superior mesenteric artery. Reglonal blood flow was monitored, using miniaturized Doppler flowprobes (Haywood et al ", 1981). To avold suppression of these chemoreflexes by anesthesia (zimpter et al.., 1981; Smits and Brody, 1984) experiments were performed in consclous unrestrained animals. For afferent nerve stimulation we selected three agents. Bradykinin (BK) was chosen since this drug is known to excite both renal and mesenteric afferents (Smits and Brody, 1984; Lew and Longhurst, 1986; Longhurst and Dittman, 1987; Weaver et al., 1987; Orduay et al." 1988). Adenosine (ADO) was applied to examine whether this agent would specifically activate renal afferents, as has been suggested by Katholl et al. (1983, 1987). 5-Hydroxytryptamine (5-HT) was tested for its ability to elicit cardiovascular reflexes which presumably could be specific for the visceral afferents since this substance is present in relatively large 
amounts in the intestines (Gershon and Erde, 1981). Furthermore, these agents were selected because their half-lives in blood are relatively short so that systemlc effects are minimized. The results suggest that activation of renal as well as visceral afferents evokes, in general, similar hemodynamic reflexes.

\subsection{Experimental protocols}

Experiments were performed on male Wistar rats, weighing $250-300$ grams. Rats were surgically instrumented in two stages.

First, cannules were inserted into the right suprarenal artery and a side branch of the superior mesenteric artery for ir and im infusions respectively. In the same operation, miniaturized pulsed Doppler probes were placed on the right and left renal artery, the superior mesenteric antery and abdominal aorta, distal to the iliolumbar arteries for regional blood flow measurements. After this surgery the animals were allowed to recover for 4 -5 days to regain their pre-operative body weight. In the second stage of surgery" catheters were implanted for iv infusions and for blood pressure measurements. All surgical details are described in chapter 2. Animals were allowed to recover overnight and experiments were started the next day.

Iv, ir and Im infusions were given in random order. The vehicle and test solutions of $B K$ and ADO were also applied in random order. 5-HT was tested in the last place since this drug frequently caused diarrhoea when infused in (Fasth et al., 1983).

The concentrations of the test solutions were for $\mathrm{BK} 50 \mu \mathrm{g} / \mathrm{ml}, \mathrm{ADO}$ $500 \mu \mathrm{g} / \mathrm{ml}$ and $5 \mathrm{HT} 500 \mathrm{\mu g} / \mathrm{mn}$. In random order, 4 infusion rates $(2,6,20$, $60 \mu 1 / \mathrm{min}$ ) were used to deliver for BK $0.1-3 \mu \mathrm{g} / \mathrm{min}$ and for $\mathrm{ADO} 1-30 \mu \mathrm{g} / \mathrm{-}$ min. For 5-HT only 3 dosages were applied $(1-10 \mu \mathrm{g} / \mathrm{min})$. Pilot studies showed that the infusion rates do not disturb renal and mesenteric blood flow. The maximal rate $(60 \mu 1 / m i n)$ is only approximately $1 \%$ of normal blood Elow. Infusions were condwcted for 5 min after which at least $10 \mathrm{~min}$ were allowed to elapse before another infusion was started.

MAP, HR and regional flows were digitized and sampled "on-line" every second and displayed. Mean values over $6 \mathrm{~s}$ were stored on disc for later analysis. With computer assistance, changes in MAP, HR, flows and calculated vascular resistances were averaged $2 \mathrm{~min}$ before infusion and the last 3 min during infusion. Flow changes were expressed as percentage change in 
$\mathrm{KHz}$ Doppler shift.

BK (Sigma, St. Louis, MO, USA) was used as the tri-acetate salt, ADO (Sigma) as free base and 5-HT (Janssen Chimica, Belgium) as creatinine sulphate monohydrate. Infusion rates are expressed as rates for the salt" if applicable.

Multiple comparisons of hemodynamic values between and within groups were subjected to analysis of variance, whereafter modified t-tests (Bonferroni) were applied (see section 2.10 ).

SALINE
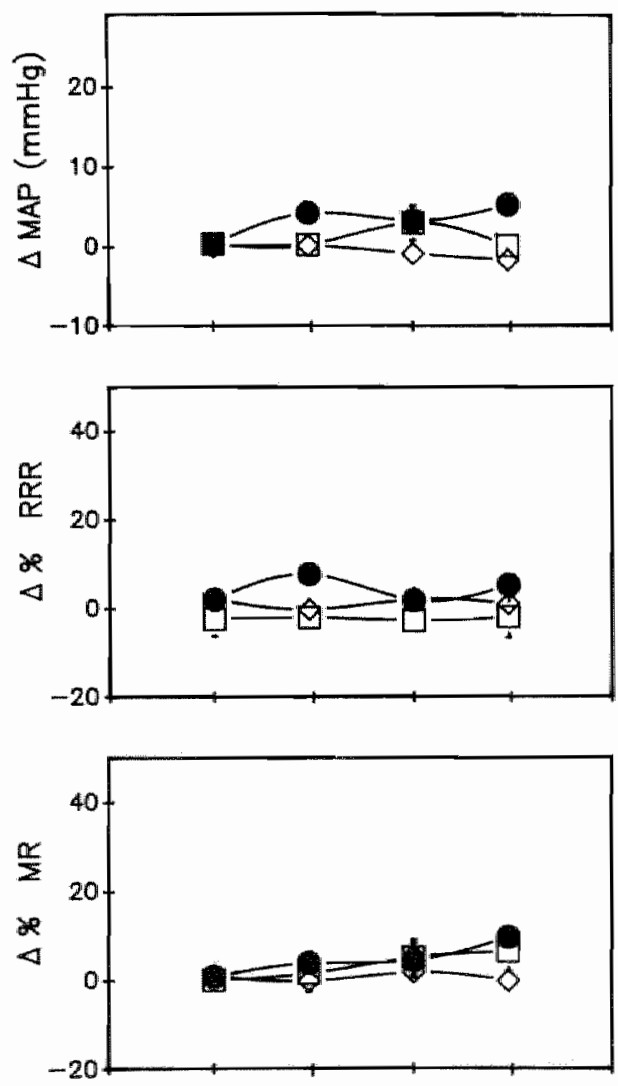
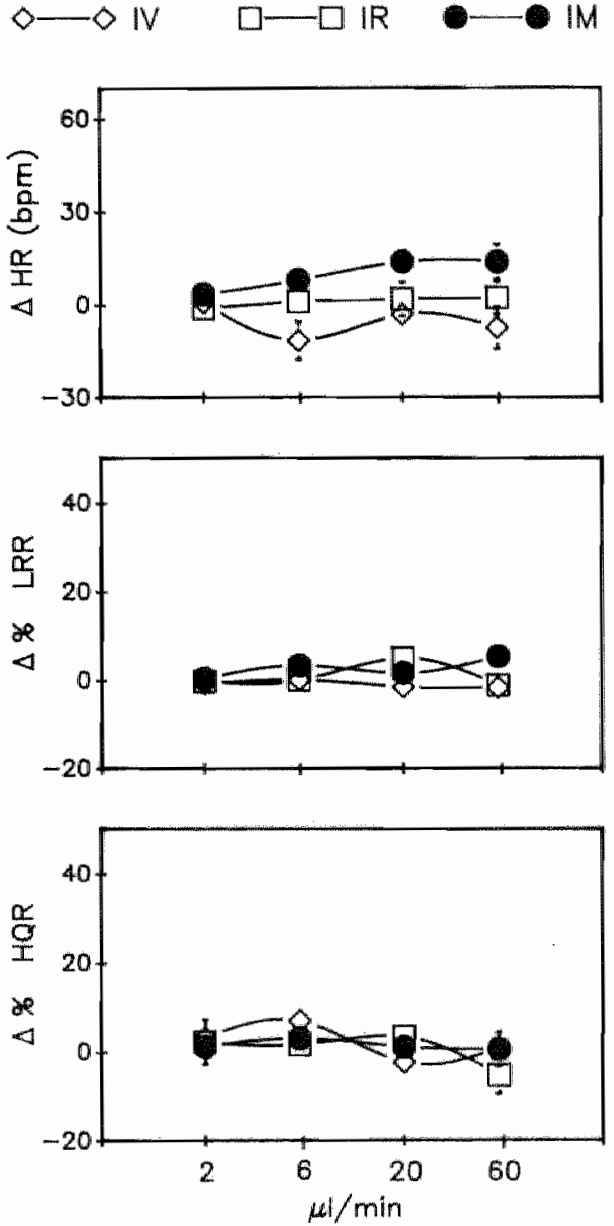

Fig. 4.1. Changes in hemodynamic parameters during iv, 1 r and im of vehlele $(0.9 \% \mathrm{NaCl})$ infusions. 


\subsection{Results}

\subsubsection{Effects of saline}

During the saline infusion experiments mean baseline values for MAP and $11 R$ were $122 \pm 2 \mathrm{mmHg}$ and $323 \pm 2$ beats/min respectively. The results of iv, it and in saline infusion for MAP, HR and vascular resistances are presented in fig. 4.1. These data show that vehicle infusions resulted only in minimal changes in hemodynamic variables. ANOVA revealed no significant differences between the effects caused by iw, ir and in saline infusions.

Table 4.1. ANOVA table comparing the hemodynamic effects caused by infusions of BK, ADO and 5-HT versus those caused by vehicle $0.9 \%$ NaCl) infusions.

Significant p< values are presented; NS: not significant.

\begin{tabular}{|c|c|c|c|c|c|c|c|c|c|}
\hline \multirow{2}{*}{$\begin{array}{l}\text { Drug } \\
\text { Infusion } \\
\text { route }\end{array}$} & \multicolumn{2}{|c|}{ Bradykinin } & \multirow[b]{2}{*}{ in } & \multicolumn{2}{|c|}{ Adenosine } & \multirow[b]{2}{*}{ in } & \multicolumn{3}{|c|}{ 5-Hydroxytryptamine } \\
\hline & $\tan$ & in & & iv & ir & & iv & in & $1 m$ \\
\hline$\triangle M A P \%$ & $\%$ NS & 0.001 & 0.001 & 0.01 & 0.05 & 0.01 & 0.001 & NS & 0.05 \\
\hline$\Delta A R \quad \%$ & $\% \quad 0.001$ & 0.001 & 0.001 & 0.002 & 0.001 & $\mathbb{N S}$ & 0.001 & 0.05 & 0.01 \\
\hline$\triangle L R F \%$ & $\% \quad 0.02$ & 0.002 & 0.001 & NS & 0.05 & 0.05 & NS & NS & 0.05 \\
\hline$\triangle \mathrm{ARRF}$ & $\%$ NS & 0.02 & 0.002 & 0.001 & NS & NS & NS & 0.01 & NS \\
\hline$\triangle \mathrm{HOF} \%$ & $\% 0.001$ & 0.001 & 0.001 & NS & NS & NS & $\mathrm{NS}$ & NS & 0.05 \\
\hline$\Delta M F \quad \%$ & $\% \quad 0.001$ & 0.001 & 0.001 & 0.002 & NS & 0.05 & NS & NS & 0.01 \\
\hline$\triangle \mathrm{L}$ RR $\%$ & $\% \quad 0.01$ & 0.001 & 0.001 & 0.05 & 0.05 & 0.01 & 0.05 & NS & 0.05 \\
\hline$\triangle R R R \%$ & $\%$ NS & 0.001 & 0.001 & 0.002 & NS & 0.01 & 0.01 & 0.01 & 0.05 \\
\hline$\triangle H Q R \quad Y$ & $\% \quad 0.001$ & NS & NS & NS & NS & NS & 0.001 & NS & NS \\
\hline$\triangle M R \quad \%$ & $\approx \quad 0.001$ & 0.001 & 0.001 & 0.001 & NS & 0.01 & 0.01 & $\mathrm{NS}$ & 0.01 \\
\hline
\end{tabular}

\section{3 .2 Effects of agents}

Table 4.1 presents the results of ANova in which the effects of the BK, $\mathrm{ADO}$ and 5-HT test solutions are compared with the effects evoked by saline infusions. From this table it may be concluded that the hemodynamic changes induced by these agents are for most parameters significantly different from values reached during vehicle infusion. The specific hemodynamic effects caused by these chemicals when infused $i v$, ir and im are presented in detall below. 


\subsubsection{Effects of bradykinin}

The results are summarized in fig. 4.2. Mean baseline values for MAp were $120 \pm 2 \mathrm{mmHg}$ and for HR $317 \pm 3$ beats/min. These values were not different from the baseline values during the saline infusions. Iv infusion of BK had no effect on MAP while HR and regional flows increased significantly as compared to values obtained during vehicle infusions (see table 4.1). The effects of IV BK on LRF and RRF were considerably less than the effects on $\mathrm{HOF}$ and $\mathrm{MF}$ which increased dose dependently by $18 \pm 3 \%$ and $39 \pm 6 \%$ at $3 \mu \mathrm{g} / \mathrm{min}$ respectively. Consequently, HOR fell $10 \pm 3 \%$ and $M R 23 \pm 3 \%$, most likely as a

BRADYKININ
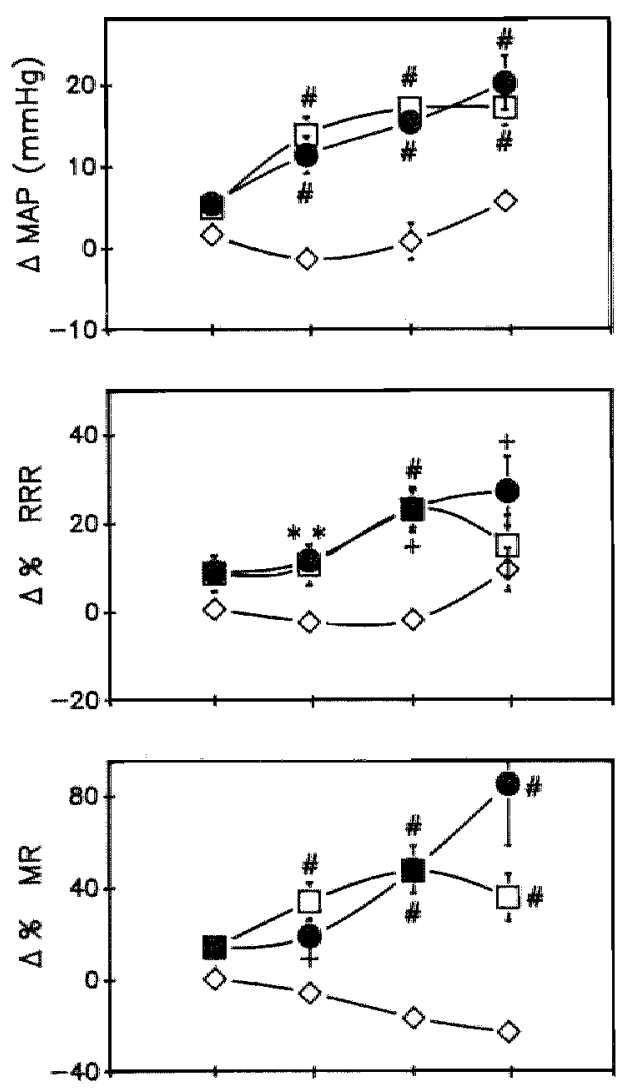

$\diamond-\infty \quad \mathbb{V} \quad \square$ IR
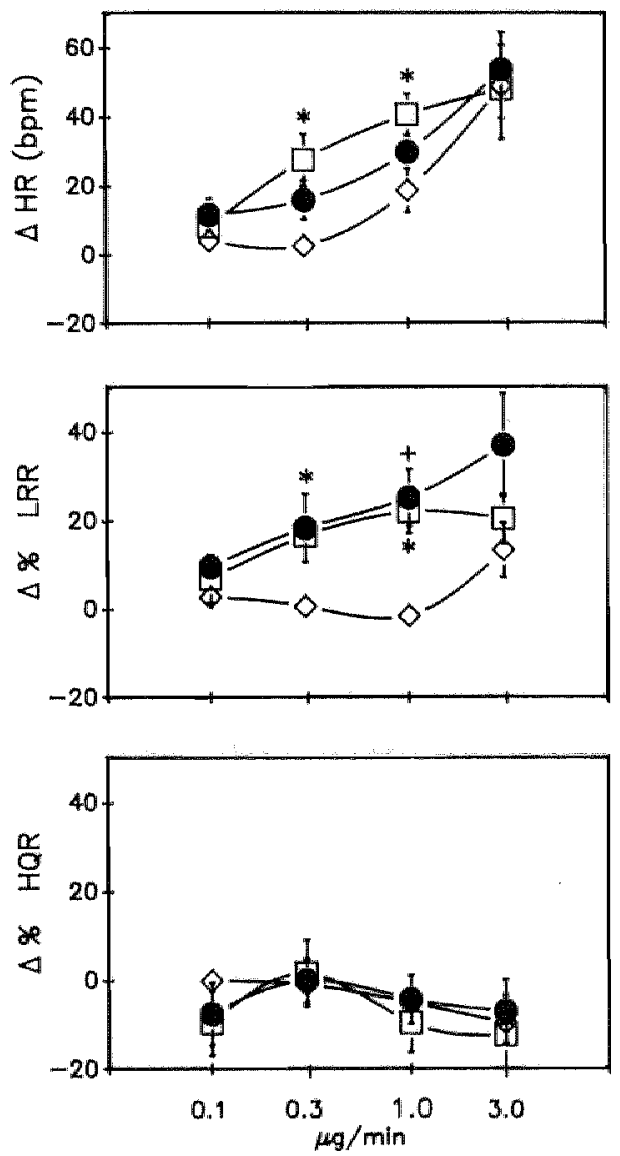

Fig. $4{ }^{2} 2$ Changes in hemodynamic parameters during iv, ir and in infusions of bradykinin.

Sigmificantly different from iy: *p<0.05, +p<0.01, \#p<0.001. 
result of ditect vasodilatory effect of $B R$.

In contrast to these effects, both ir and in infusion of $B$ K caused even at dose of 0.3 ug/min a significant rise of MAP and HR when compared to values observed during vehicle infustons or $i$ V BK Infusion. An example of a rypical recording during im infusion of BK is shown in $\mathrm{fig} .4 \mathrm{~m}$.

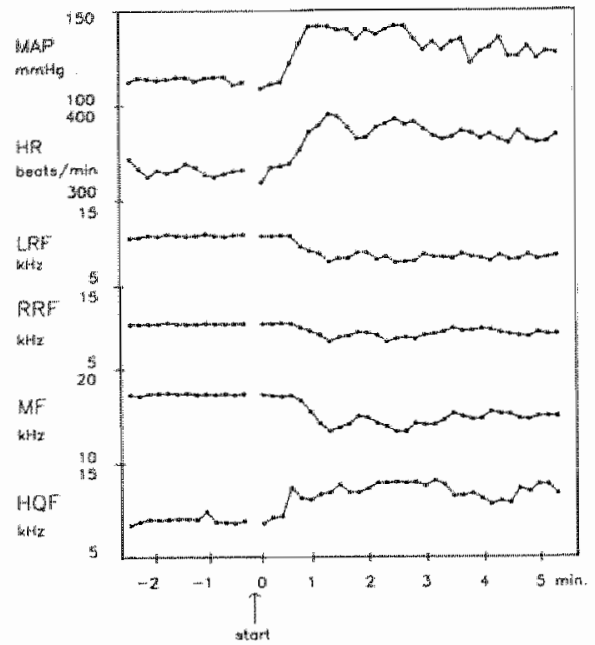

Fig. 4.3 Typical computerized recording during a 5 min intramesenteric infuston of $B K(1 \mu g / m i n)$.

During ir infusions, LRF decreased maximally $8 \pm 3 \%$, RRF $6 \pm 3 \%$ and MF $18 \pm 4 \%$ while in contrast HOF rose maximally $46 \pm 18 \%$. As a result, LRR, RRR and MR increased considerably while HOR fell maximally $12 \pm 9 \%$. As depicted In $\mathrm{flg} .4 .2$, essentially the same hemodynamic pattern was evoked during im BK application at the same infusion rates. Differences between the effects elicited by $x$ and im infusion were not statistlcally signficant. However, for both infusion routes, the efects on MAP, LRR, RRR and MR were significantly different $(p<0.01)$ from values during iv infusions.

\subsubsection{Effects of adenosine}

Mean baseline values during ADO infusions for MAP were $124 \pm 2 \mathrm{mmHg}$ and for $\mathrm{HR} 316 \pm 3$ beats/min, which were not statistically different from values during saline infusions. The effects of ADO infusions are presented in fig. 4.4. ADO infused iv decreased MAP significantly as compared to saline infusions (table 4.1). Flows through most vascular beds increased and conse- 
quently, regional vascular resistance decreased which was most pronounced at maximal infusion rates in the renal and mesenteric vascular bed (LRR $-10 \pm 3 \%, \operatorname{RRR}-12 \pm 2 \%$ and MR $-16 \pm 3 \%$ ).

In contrast, ir and in ADO infusion caused simllar dose-dependent rises in MAP which vere significantly different from vehicle as well as iv ADO infusion. These increases in MAP were accompanied by small increments in $\mathrm{ER}$, which were not different from the values during iv infusion of the same amount.

\section{ADENOSINE}
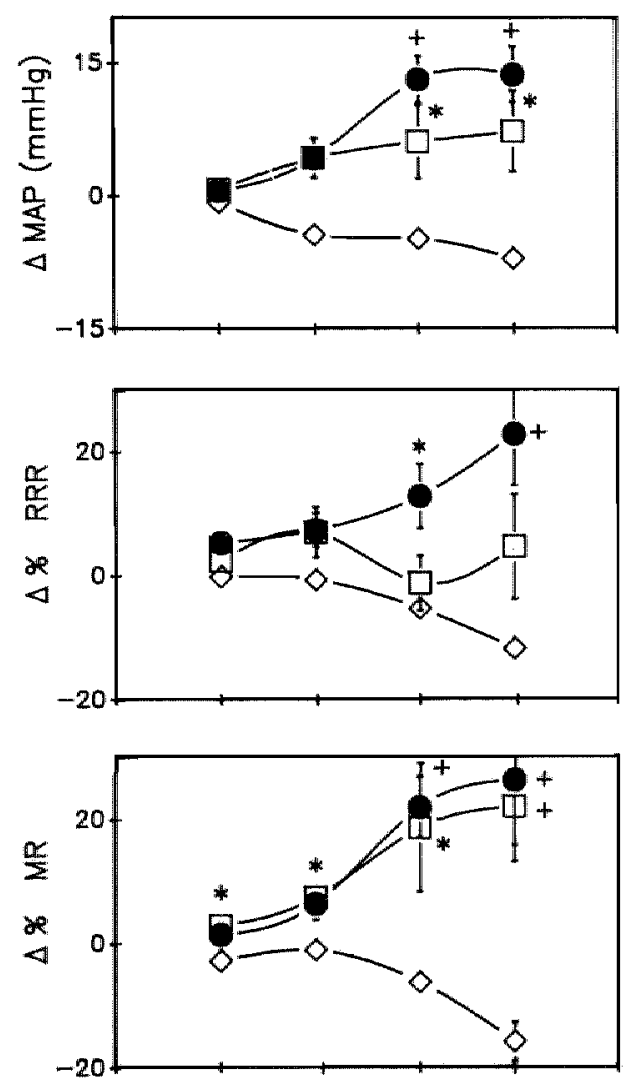

$\diamond-O \mathrm{IV} \quad \square-\square \mathrm{IR} \quad-\mathrm{IM}$
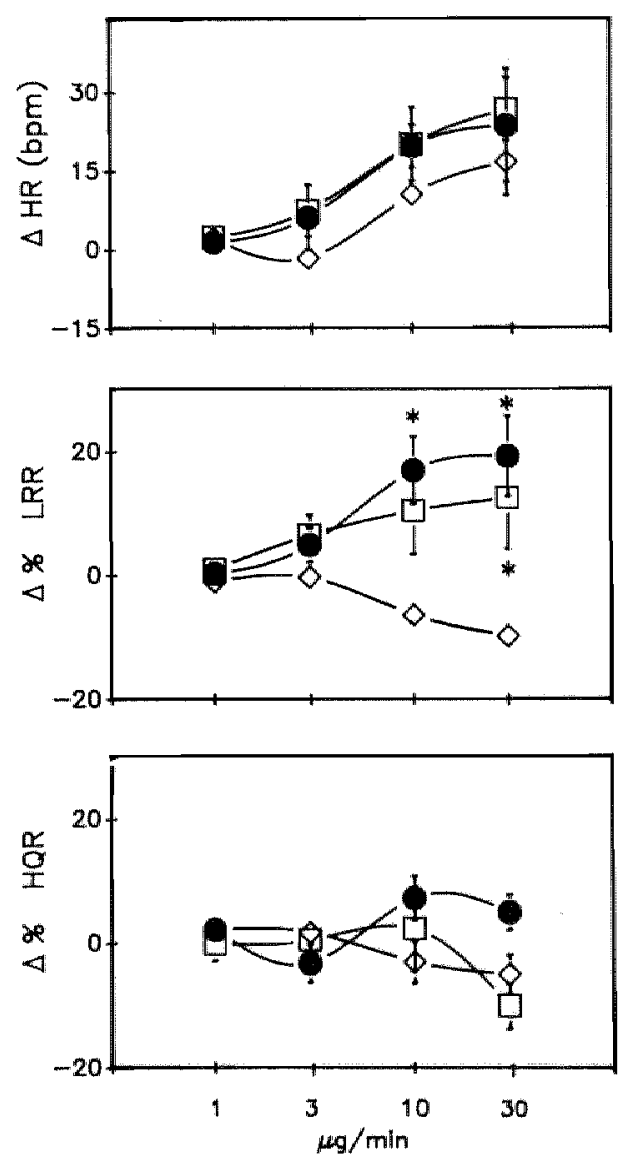

Fig. 4.4 Changes in hemodynamic parameters during $1 \mathrm{w}$, it and im adenosine infusions. Significantly different from iv: *p<0.05, +p<0.01. 
Local ADO infusion caused no vasodilation of the target organ. Flows were unchanged or if anything even decreased during ir or in ADo infusion. Remarkably, in the mesenteric vascular bed even a $30 \mu g / m i n$ ADO infusion decreased WP by $10_{4} 5 \%$ while during iv infusion of the same amount HF rose $11 \pm 4 \%$. According to these results, LRR, RRR and MR rose to levels that are significantly different from values reached during vehicle infusions as well as 1. ADO infusions. These vasoconstrictor effects were not significantly different beteen the mesenteric and renal vascular bed. No changes in HQR due to ADO infusion were observed.

5 HYDROXYTRYPTAMINE<smiles>O=[18O]</smiles>
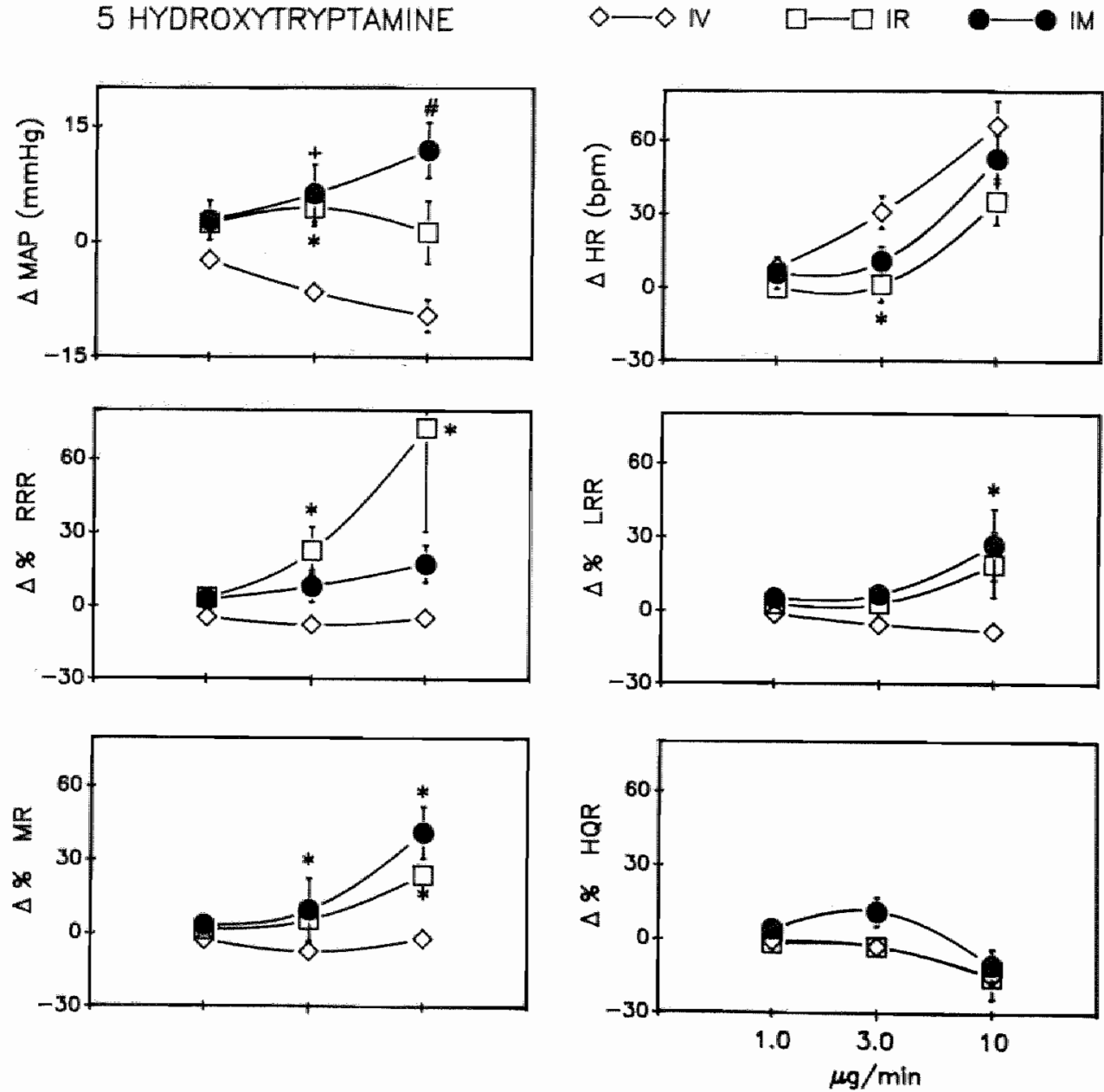

Fig. 4.5 Changes in hemodynamic parameters during iv, ir and im infusions of 5-hydroxytryptamine. Significantly different from iv: ${ }^{\star} p<0.05$, $+p<0.01$, \#p<0.001. 


\subsubsection{Effects of 5-hydroxytryptamine}

Mean baseline values for MAF were $122 \pm 2 \mathrm{mmHg}$ and for HR $310_{ \pm 4}$ beats/ min during the 5-HT infusion experiments. These values vere not different from those during the vehicle solutions. Iv infusion of 5-hit decreased MAP significantly as compared to vehicle infusions (see table 4.1). Most regional flows did not change, resulting in small but significant decreases in resistances in all vascular beds when compared to vehicle infusions. HR increased markedly during the iv 5-HT infusions. Ir infusion of 5-HT was without effect on most hemodynamic parameters except for HR which was increased (see fig. 4.5). Furthermore, vascular resistance rose dose dependently in the infused kidney.

Im infusion increased MAP and HR signiflcantly above levels observed during im saline infusions and iv 5-HT infusions, LRF and RRF decreased by $9 \pm 5 \%$ and $4 \pm 4 \%$ respectively and LRR and RRR rose significantly (as compared to vehicle infusions) by $27 \pm 14 \%$ and $17 \pm 8 \%$ during $10 \mathrm{\mu g} / \mathrm{ml} \mathrm{im}$ 5-HT. This infusion rate also caused a $20 \pm 5 \%$ decrease in MF and a $41 \pm 11 \%$ significant increase in MR.

During the ir and in infusions no significant differences between changes in mesenteric and renal vascular beds were observed. HoR did not change at any 5-HT infusion rate.

\subsection{Discussion}

The goal of the present study was to examine whether sensory nerveevoked hemodynamic reflexes from the kidney and mesentery have organ specific characteristics. This hypothesis implies that there may be special renal and mesenteric receptors which could elicit a unique hemodynamic reflex for that organ. The question was addressed by comparing hemodynamic reflexes evoked by local ir and im infusions of 3 different chemoreceptor stimulants.

The present data show that in conscious unrestrained rats, intraarterial infusion of chemoreceptor stimulants into the renal and mesenteric vascular beds causes a reflex pattern of hemodynamic changes, which is remarkably different from the pattern observed when these agents are applied systenically. This finding suggests that the effects during local intra-arterial infusion depends on the activation of a fast acting mechanism originating from the target organs. There is an abundance of evidence from electrophysiological and denerwation studies (Otdway et al., 1983; 
Ordway and Longhurst, 1983; Sinits and Brody;, 1984; Golin et al., 1987; Kathol1 and Woods, 1987; Tobey and Weaver, 1987; Weaver et a1., 1987; Ordway et al., 1988; Stein and Weaver, 1988) in support of the hypothesis that these hemodynamic changes are the result of activation of local sensory nerves. Furthermore these studies provided evidence that the hemodynamic changes upon afferent stumulation are caused by an activation of the sympathetic nervous system. However, care should be taken to interpret the changes in organs in which the agents are infused, since vascular reactions in these organs are the result of the sumation of direct vascular effects and nerve reflexes.

Stimulation of renal and mesenteric afferents wth BK, ADO and 5-HT induced in general a consistent hemodynamic reflex pattern characterized by a dose-dependent increase in MAP, HR and resistance in the renal and mesenteric vascular beds, while the hindquarter vascular bed behaved passively. As compared to ADO and 5-HT, BK was the most effective agent in eliciting these reflex reactions. The peptide induced significantly greater maximal hemadynamic changes during $i r$ as well as $1 \mathrm{~m}$ infusions even at doses 10 times lower than those for $A D O$ and 5-HT. Intra-arterial infusion of ADO into the mesenteric vascular bed resulted in similar dose-dependent reflex responses as ir infusion of ADO. This suggests that there is no role for ADO in eliciting unique reflexes via stimulation of ADo sensitive renal afferent nerves located in the renal pelvis (Katholi and Woods, 1987). stimulation of visceral afferents with 5-HT did also not lead to characteristically different sympathetic reflexes as compared to the results achieved with ADO. Yet, in the present experiments, the effects of im infusion of 5-HT were significantly greater than during it infusion. Nevertheless, we suggest that this does not reflect a unique role for 5-HT in inducing visceral afferent reflexes, but rather an artifact. In contrast to tenal tissue, the gastro-intestinal tract contains a large amount of 5-HT which is metabolized at characteristlcally high rates for this tissue (Gershon and Erde, 1981). Thus, during high intra-arterial infusion rates, less 5-HT shall leak into the systemic circulation via the im than via the Ir route and consequently less systemic effects of 5-HT will mask its effect as a chemoreceptor stimulant. The strong vascular constriction obserwed during ir as well as im infusion of 5-HT in the respective infused organs is probably due to stimulation of local $5-\mathrm{HT}_{2}$ receptors (Bradley et al., 1986). The chemoreceptor stimulatory effect of 5-HT is supposed to be expressed by $5-\mathrm{HT}_{3}$ receptor stimulation (Bradley et al., 1986). The experiments, however, were not designed to discriminate between receptor types. 
The present data showed that neither application of different chemicals nor their route of infusion did result in qualitatively different hemodynamic reflex patterns. Moreover, the fact that all ir and im induced responses were dose dependent and differed only in magnitude but not in pattern For BR, $A D O$ and 5-HT suggests: a) that the amount and/or intensity of afferent input determines the degree of sympathetic actuvation and $b$ ) that no specific sympathetic reflex pathways for renal and visceral afferents exist. In all cases there was a consistent rise in MAP and HR, combined with greater increases in MR than RR, indicating not that the afferent input determines the hemodynamic reflex pattern of sympathetic stimulation, but that this pattern is rather a predetermined reflex pathway that can be activated to several degrees.

The present hemodynamic reflexes may be organized in spinal sympathetic functional units as described by Jänig (1986). In this framework renal and wisceral induced hemodynamic reflexes may share common reflex pathways. This could imply convergence of renal and visceral afferent input as suggested by the work of Ammons (1986, 1987).

Another factor which may contribute to the regulation of expression of these reflex responses is the possibility that during im infusion of the agents not only sympathetic but also vagal afferent nerves are stimulated. Several authors showed that reflex responses upon stimulation of intestinal receptors were increased in vagotomized cats (Tobey and Weaver, 1987; Weaver et al., 1987). Thus, concomitant activation of vagal afferent nerves might suppress the sympathetic pressor responses. However, since 1 it is assumed that in contrast to the intestines the kidney has no vagal innervation (Norvell and Andersion, 1983) the present data suggest that these vagal visceral afferent réflexes are not involved. Alternatively, assuming that these vagal reflexes do play a role in these experiments, this would imply that the kidneys possess a vagal afferent innervation as proposed by Gattone et al. (1986). For the moment, this question remalns unresolved.

Probably, the most important determinant in the expression of the pattern of the sympathetic reflex responses is the baroreflex. Baroreflex control of sympathetic outflow to the different vascular beds is non-uniform. Several reports have indicated that baroreflex out flow to the renal vascular beds is significantly more enhanced than to the mesenteric vascular bed (Nievelstein et al., 1986; Tobey and Weaver, 1987; Weaver et al., 1987; Meckler and Weaver, 1988; Stein and Weaver, 1888). In the present study baroreflexes seem to be overruled since HR is elevated during the afferent nerve dependent sympathetic pressor responses. Mevertheless, the 
increase in RR which is in most cases significantly smaller than in MR upon afferent stimulation may depend upon a larger restraint of baroreflex outflow to the renal than to the mesenterlc vascular bed.

In contrast to the above mentioned arguments several observations have provided evidence for the existance of unique renal and mesenteric reflexes. ARN-evoked renorenal reflexes (Gollin et al., 1987), which are defined as responses elicited in one kidney as a result of interventions on the siame or opposite kidney, can be qualified as such. They are evoked by changes in the fonic composition of the renal pelvis or by raising ureteral pressure (Kopp, 1985). These stimuli are assumed to be very specific for the kldney (Moss, 1982; Kopp, 1985). Such unique functional reflexes have not been demonstrated yet to be elicited from the visceral afferent nerves. However, the variety in the hemodynamic responses upon stimulation af the sensory nerves from several of visceral organs (Tuttle and Mccleary, 1975; Fasth et al., 1983; Ordway et a1., 1983; Ordway and Longhurst, 1983; Longhurst et a1., 1984; Lew and Longhurst, 1986; Longhurst and Ditman, 1987; Tobey and Weaver, 1987; Weaver et al., 1987; Heckler and Weaver, 1988; ordway et al., 1988; Stein and Weaver, 1988) might indicate their existence. Since the visceral organs have important metabolic and vegetative functions, their sensory nerve endings may be sensitive for certain metabolites such as glucose (Niijima, 1983), resulting in autonomic reflexes which contribute to these metabolic and vegetative functions. In this respect, BK, $A D 0$ and 5-HT which were used in the present study, apparently have no selectlve affinity for these renal and mesenteric receptors. Since these agents may excite several populations of afferent fibers (Belcher, 1979; Gershon and Erde, 1981; Lew and Longhurst, 1986) the observed hemodynamic effects are most likely the result of a summation of such reflexes.

This leads us to the question whether the BK, ADO and 5-HT induced renal and visceral afferent nerve dependent cardiovascular reflexes have functional signfficance. In some reports, the endogenous substances ADo, 5-HT and especially BK have been implicated to be released and to mediate pain in several renal and gastro-intestinal diseases (Ammons, 1986; Lew and Longhurst, 1986). However, in the present experiments, renal and visceral reflexes could be initiated in the absence of pain. Several recordings were made while rats were and stayed asleep during local infusions. In parallel Wth our observations, Pagani et al. (1985) described painless sympathetic pressor reflexes upon Intracoronary infusion of BK in conscious dogs. Pain reactions vere only apparent when the recovery from surgery was not complete. Remarkably, intracoronary application of BK was then associated wth 
depressor effects. In addition, Faber and Gettes (1987) shoved that similar ARN dependent renal pressor reflexes cam be elleited in conscious intact and spinal transected rats. Taking all data together, $1 t$ is clear that these renal and visceral afferent nerve dependent reflexes can be restricted to spinal levels only and occur in the absence of pain.

The most important role for these sensory nerte induced hemodynamic reflexes is probably their contribution to cardiovascular homeostasis. It has been speculated that afferent merve dependent reflexes may play a role in blood pressure maintenance during hypotensive shock (Longhurst and Dittman, 1987). Since in the latter state local release of BK and ADO is likely to occur, afferent nerve dependent reflexes as described in this study may be elicited to increase blood pressure. Afferent renal nerve dependent sympathetic pressor mechanisus are known to be activated when blood flow through the renal arteries is intentially reduced (Barber and Moss, 1985; Faber and Gettes, 1987; Katholi and Woods, 1987). In additton, Wyss and co-workers (1986) have shown that these sympathetic pressor responses con.. tribute importantly to the initiation and maintenance of renal hypertension in 1-clip 1-kidney rats. It is unknown whether visceral afferents increase their activity when perfusion pressure is gradually reduced as renal afferents do (Barber and Moss, 1985). However, a recent electrophysiological study has shown that visceral sensory nerves may become activated during ischemic and hypoxic conditions (Longhurst and Dittman, 1987). Since in the present experiments im infusions of chemoreceptor sitimulants evoked similar pressor reflexes as during stimulation of renal afferents, it is tempting to speculate that during flow reductions in the mesenteric artery, substances are released wich initiate pressor reflexes to increase perfusion pressure. When this is prohibited in pathological conditions such as atherosclerosis, these local reflexes may also become chronically active and may thus contribute to the induction of hypertenstion. Whether these mechanisms are only restricted to the renal and mesenteric vascular beds is still unknown. However, several reports indicate that similar mechanisms play a role in in the cardiovascular changes observed during myocardial ischemia (Pagani et al., 1985; Heusch et all., 1985) or compression of the hindlimb (Osterziel et al., 1984).

Thus, in a physiological sense, one function of these afferent nerve dependent hemodynamic reflexes may be their ability to increase perfusion pressure under ischemic conditions. The exact nature of the stimulus remalins uncertain. $B K$ and $A D O$ are endogenous substances that have been proposed to be released in response to oxygen deficiency. It is belleved that 
through their vasodilator action, blood flow returns towards normal. However, the present data are not campatlble with this theory for reasons explained above. The described autonomic hemodynamic reflexes may contribute to the pressure seeking properties of the central nervous systen, which 1 tem has been recently revieved by Julius (1988).

In conclusion, stimulation of renal and mesenteric afferents by local Intra-arterial infusion of $B K$, $A D O$ or 5 -HT evokes a general pattern of hemodynamic reflexes which suggests that these afferents share not only common reflex pathways but also a common physiological function. Under certain conditions, these sensory reflexes may contribute to the initiation or maintenance of hypertensive mechanisms. 


\subsection{Introduction}

In the introduction to this thesis, it has been postulated that increased ARN activity may be a mechanism through wich hypertension can be initiated. Indeed, as shown in the previous chapter, intrarenal infusion of chemoreceptor stimulants reflexly increases blood pressure in consclous rats. Up to now, the hypertensive effects upon ARN stimulation have only been studied for very short periods of time. The acute sympathetic activation induced no long lasting hemodynamic effects. Blood pressure returned to control levels within a few minutes after the intrarenal infusions were stopped.

The aim of the present study was to examine the ARN dependent hemodynamic reflex responses induced by a prolonged intrarenal infusion of bram dykinin in conscious rats. Experiments were performed in renal deafferented rats and sham operated rats to evaluate whether other factors than ARM are involved in the hemodynamic changes. To prevent the occurrence of tachyphylaxis to BK, also intermittent infusions were applied.

Experiments were performed in rats with either one or two kidneys to examine the role of the contralateral kidney in the ARN dependent reflex responses. Faber (1987) has demonstrated that the opposing kidney might release humoral factors which antagonize the ARN dependent pressor response induced by renal flow reduction. BK was continuously infused in SHR and WKY rats to evaluate whether ARN dependent reflex mechanisms might be altered in SHR as has been suggested by Kopp (1987). Furthermore the role of prostaglandins in the BK induced hemodymamlc reflexes was studled. It has been shown that prostaglandins may modulate the sensitivity of peripheral afferent nerves to several factors (Ohno et al. ", 1983).

\subsection{Experimental protocols}

Al1 rats were implanted with catheters for blood pressure measurements and iv and ir infusions as described in chapter 2. On the experlmental days, rats were placed in normal experimental cages. After a stabil1zation period of $1 \mathrm{hr}$, infusions were started. 
In random order, ir or $1 \mathrm{v} \mathrm{BK}(1 \mathrm{\mu g} / \mathrm{min})$ or vehicle $(0.9 \%$ Hacl 10 Hl/min) infusions were started and maintained for 5 to $7 \mathrm{hr}$. Solutions were refreshed every $2 \mathrm{hr}$. MAP and HR were monitored continuously (see chapter 2). The following experiments vere performed:

1. BK was continuously infused ir in sham operated or renal deafferented (method for ARN- $x$ is described in chapter 2) WRY rats with one-kidney only.

2. Intermittent (10 min on/off) it or it (control) infusions of BK were applied in intact two kidney why rats for 7 hours.

3. BK or saline (control) were continuously infused ir in SHR and WKY rats: with both kidneys intact.

4. In intact WKY rats, acute $(2-5$ min) MAP and HR responses were studied durlng a 5 min it $B K$ infusion $(0.3$ and $1.0 \mu g / m i n)$ before and after application of the prostaglandin synthesis inhibitor indomethacin ( 5 $\mathrm{mg} / \mathrm{kg}$ ) or its vehicle solution. Indomethacin (Sigma) was dissolved in $10 \% \mathrm{Na}_{2} \mathrm{CO}_{3}$ in saline and injected (100 $\left.\mathrm{Hl} \mathrm{iv}\right) 15 \mathrm{~min}$ before the start of the in $\mathrm{BK}$ infusion.

\subsection{Results}

The hemodynamic effects of a $4 \mathrm{hr}$ continuous is BK infusion in $A R M-X$ and sham operated rats are presented in fig. 5.1. In the sham control animals, MAP increased immediately and stayed significantly elevated above pre-infusion levels for at least $1 \mathrm{hr}$. In ARN-x rats no rise in MAP was observed during the infusion period. In control rats, HR was slightly increased during the first hour of ir infusion of BK. However, no significant differences between ARN-X and sham control rats were present.

Flg. 5.2 1llustrates the effects of a $5 \mathrm{hr}$ continuous ir BK infusion in intact SHR and WKY rats. In both groups MAP rose significantly for apm proximately $2 \mathrm{hr}$ above levels reached during the vehicle infusions. Remarkably, increases in HR were significantly (ANOVA, $p<0.01$ ) more pronounced in the SHR than in the WKY group during the infusion period.

Throughout the experiment the intermittent $(10 \mathrm{~min}$ on/off) ir $\mathrm{BK}$ infusion caused a characteristic hemodynamic pattern as shown in fig. 5.3. MAP was significantly increased above control levels during each period of infusion. Values of MAP during the periods of non-infusion were not signiflcantly different from the pre-infusion values. The effects on HR during the intermittent ir infusions were comparable with those for MAP, although 
CONTINUOUS INTRARENAL BRAIDKKININ INFUSION
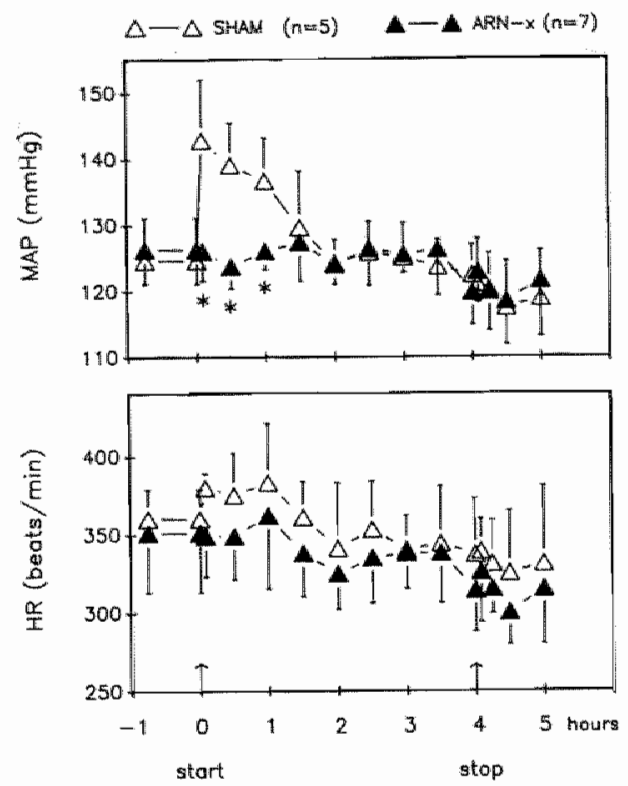

Fig. 5.1

Hemodynamic effects of a 4 hour continuous ir BK infusion ( $1 \mu \mathrm{g} / \mathrm{min}$ ) in ARN-X and sham operated WKY rats. Significantly different from sham: *p<0.001.
CONTINUOUS INTRARENAL BRADYKININ INHUSION
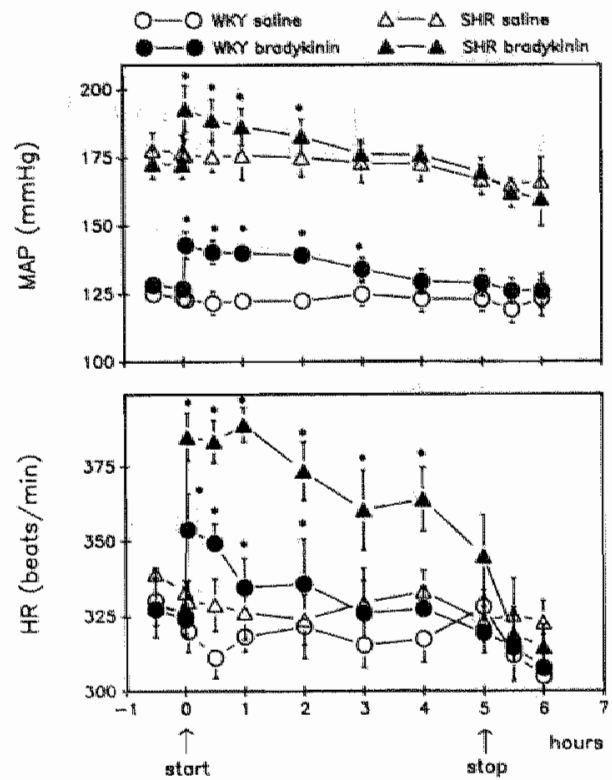

Fig. 5.2

Hemodynamic effects of a 5 hour continuous ir BR infusion (1 $\mathrm{\mu g} /$ min) in SHR $(n=5)$ and WKY $(n=5)$ rats. Significantly different from saline: $* p<0.01$.

less pronounced. A minor $\mathbb{E a d}$ in MAP and HR was the result of the intermittent iv BK infusion.

Pretreatment with saline, vehicle or the indomethacin solution did not influence basal MAP which was $120 \pm 4,123 \pm 3$ and $122 \pm 2$ mim Hg respectiveIy. After indomethacin pretreatment, basal HR fell to $299 \pm 13$ beats/min, which was significantly different (ANOVA, $p<0.05$ ) from values after pretreatment with saline $\left(333_{ \pm} 10\right.$ beats/min) or the vehicle solution ( $335_{ \pm 12}$ beats/min). Pressor responses induced by both ir infusion rates of BK were significantly attenuated by pretreatment with indomethacin $(\mathbf{E} \mathbb{H}, 5,4)$. 
INTERMUTTENT GRADYKININ INFUSIONS

$10 \mathrm{~min}$ on/of $\longrightarrow \mathrm{H} \quad \mathrm{O}-\mathrm{W}$
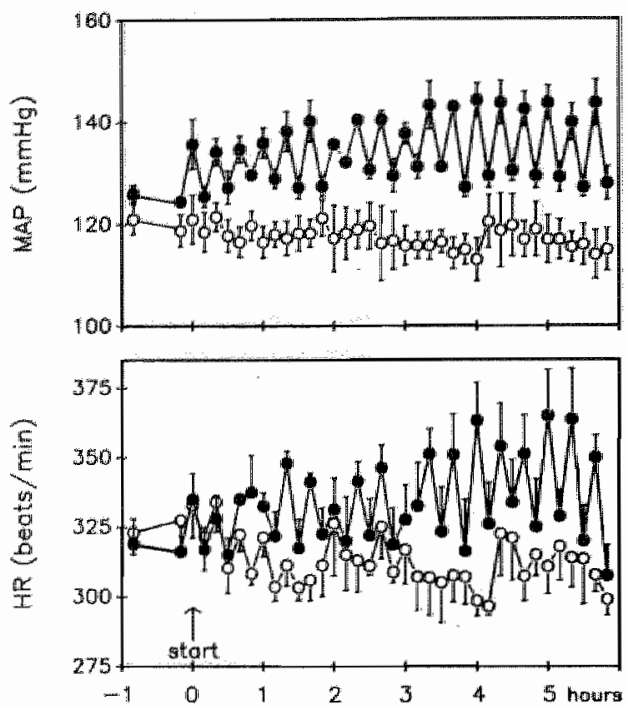

INTRARENAL ERADYKKNIN INFUSION

Effects of pretreotment
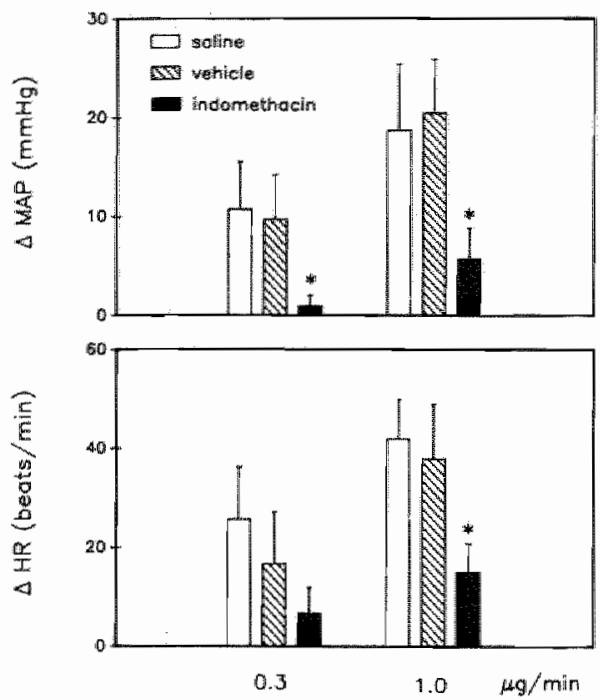

Fig. 5.3

Hemodynamic effects of a intermittent ir and $I v B K$ infusion ( $1 \mathrm{\mu g} / \mathrm{min})$ in WKY rats $(n=5)$.
Fig. 5.4

Effects of pretreatment with indomethacin $(5 \mathrm{mg} / \mathrm{kg})$ on the pressor responses elicited by ir BK infusions in conscious Wistar rats $(n=4)$. Significantly different from saline and vehicle: $* p<0.05$.

\subsection{Discussion}

This study clearly demonstrates that continuous ir infusion of $B K$ in conscious rats induces an increase in MAP and HR only via stimulation of ARN and not through other factors, since in ARN-x rats hemodynamic parameters during infusion of BK did not deviate from pre-infusion levels.

The duration of the pressor response induced by a continuous ir $B K$ infuslon was vartable, but lasted in general $1-2$ hr. Thereafter, blood pressure returned to controls levels. An similar pattern has been described by Faber (1987) who reported that the ARN dependent renal pressor reflex evolked by unilateral renal artery stenosis, was reversed within $4 \mathrm{hr}$. He suggested that this was most likely due to an as yet undefined humoral factor released by the contralateral kidney since after remowal of the latter, the reflex persisted as long as renal stenosis was sustained. In the present experiments, however, the hypertensive response declined both in rats with either one or two kidneys. These results probably reflect 
tachyphylaxis of the renal sensory neurons ta BK. In electrophysiological experiments (Ohno et al. , 1983) and hemodynamic studies (Stebbins et al., 1985) it has been shown that this phenomenon can develop relatively fast in reaction to BK. Furthermore, these studies indicated that prostaglandins are permissive factors in the $\mathrm{BK}$ induced excitation of sensory neurons. This hypothesis is corroborated by the fact that in the present acute experiments indomethacin pretreatment significantly attenuated the acute pressor responses to ir BK infusion. In addition, Faber (1987) recently showed that also the ARN dependent renal pressor reflex was virtually abolished by inhibition of prostaglandin synthesis. Thus, the decline of the BK induced pressor response may reflect local depletion of prostaglandins.

The present results indicate that in WKY rats and in SHR similar prow longed pressor responses can be evoked. Remarkably, the tachycardia evoked by the ir BK infusions was significantly greater in SHR than In WKY rats. Whether this effect is due to an increased sensitivity of the renal sensory receptors in SHR as described by Moss (1987) is unknown. These data are in sharp contrast to findings by Kopp (1987) who failed to show an increase in ARN activity upon renal mechano- and chemoreceptor stimulation in SHR, suggesting a peripheral defect at the level of the sensory receptor in these animals. It is unlikely that the differences beween our results and the results of Moss on the one hand and those of Kopp on the other hand can be explained by activation of different receptor subpopulations. According to Belcher (1979) and Lew and Longhurst (1984), BK activates large populations of chemosensitive nerves. Moreover, Protasoni et al. (1987) demonstrated that renorenal reflexes in SHR were operating normally or even slightly potentiated in comparison to WKY rats. An alternative explanation for the exaggerated tachycardia in SHR may be the characteristic impairment of baroreflex mechanisms (cf. Struyker-Boudier et al., 1982) that counteracts: this ARN induced sympathetic stimulation. In fact, the exaggerated increase in HR in SHR can even be the result of a combination of the two mechantsms proposed. On the basis of these data, it is tempting to speculate that in SHR the exaggerated hemodynamic responses to ir BK infusion may reflect ARN dependent mechanisms through which hypertension has been initiated in this model. This hypothesis will be tested in chapter 9.

Although in theories on hypertension it has been generally accepted that chronic sympathetic stimulation may initiate high blood pressure and induce permanent hemodynamic changes, up to now, only very few papers have been published in which an attempt was made to test this hypothesis. Using intermittent stimulation of the hypothalamus, a major central structure for 
cardiovascular control (Brody and Johnson, 1980), Folkow and Rubenstein (1966) Eound a significant increase in arterial pressure, that developed gradually over 14 veeks of stimulation. In contrast, Bunag and Riley (1979) falled to observe a sustalned elevation of blood pressure desplte of a persistent cardio-acceleration.

In the present experiment, an intermittent ir infusion of BK was applied to circumient the symptoms of tachyphylaxis. The results indicate that the $10 \mathrm{~min}$ tine 1 nterval was sufficlent to prevent the renal receptors from insenslbility to BK. However, the $7 \mathrm{hr}$ ARN dependent sympathetic stimulation did not alter baseline hemodynamics. After each infusion period, MAP and HR returned to levels not signiflcantly different from control levels. Yet, the time course of stimulation is probably much too short to induce permanent hemodynamic changes.

There is, however, a vast body of evidence in support of the hypothesis that sympathetic hyperactivity caused by repeated emotional or mental stress can induce hypertension. The interpretation of those cardiovascular effects is, however, hampered by the fact that most of these stimuli such as aversive operant condithoning (Friedman and Dahl, 1975) or psychosocial stimuli (Henry et al., 1975) cannot be translated into qualitative and quantitative physiological mechanisms. In addition, the effects may be remitted by habituation, a special form of adaptation in which the magnitude of responses to frequently repeated sensory stimuli decreases progressively (Buñag et a1., 1980).

The advantage of the present method in studying the long-term cardiovascular effects of sympathetic stimulation may be that the technical practicability is fairly simple and that this sympathetic reflex (as discussed in chapter 4) is most likely not relayed via supraspinal structures. Thus, changes in normal behawior or other forms of habituation do probably not hinder or counteract the mechanisms, activated by ir BK infusion.

In conclusion, long-term sympathetic stimulation via ARN can be achieved by intermittent BK infusions. This may be a mechanism to initlate hypertension. Additional studies, using stimulation periods over days to weeks, should be performed to further explore this hypothesis. 


\subsection{Introduction}

One of the most fundamental rolles of the kidney is the separation of salt and water excretion, thus allowing the body to maintain constancy of both osmolarity and composition of fluids despite wide variations of water and solute intake. The influence of efferent renal nerves on renal excretory function is readily demonstrated in experimental animals by section or by electrical stimulation of the these nerves. Acute denervation of the kidney leads to a several-fold increase in water and sodium excretion, the so-called denerwation diuresis and natriuresis. Low level electrical stimulation increases tubular sadium reabsorption in the absence of changes in renal hemodynamics. Studies in this field have been extensively revieved in recent papers (DiBona, 1982; Moss, 1983; Gottschalk et al., 1985). The influence of afferent renal nerves on renal control of excretory function is known from studies in which a change of excretory activity in one kidney (e.g. as the result of denervation) is associated with a reciprocal change in excretory activity in the other kidney such that total excretion of salt and water is unchanged or minimally affected. These so-called renorenal re-flexes have recently been reviewed by kopp (1985).

The mechano- and chemosensitive afferent renal nerves could be unique tools in normal day to day control of water and sodium balance. Their special anatomical location (Barajas et al., 1978, 1984) and thel $r$ characteristic sensory properties (see chapter 3), make it likely that these nerves are a sensitive and probably fast acting mechanism involved in the control of renal excretory function. Already minor changes in renal perfusion pressure (Barber and Moss, 1985) or small deviations tin the composition of urine (Recordati et al., 1978, 1980) may alter afferent renal nerve activity, perhaps leading to appropriate compensatory changes in efferent renal nerve activity. Although many other nervous and hormonal mechanisms are known to be involved in maintaining body fluid homeostasis (Goteschalk et al., 1985; Knox and Granger, 1987), the special feature of this mechanism is that the sensor organ is located in the kidney itself, thus allowing direct control. Unlike the stimuli which are necessary to induce changes in vasopressin or aldosteron release or baroreceptor function (Thorén et al., 1982) - 1.e. deviations in the ionic composition of the circulating bload the renal sensory nerves could already detect disturbances in the kidney 
1tself in the absence of these systemic deviations and send information to spinal as well as supraspinal centers which are involved in homeostatic control (Caverson and cirlello, 1987). Thus the sensory renal nerves may have a role in renal control of water and salt excretion through modulation of efferent renal nerwe activity. The other reflex pathways influencing efferent renal nerve actiwity (Gottschalle et al.; 1985) as well as hormonal systems may increase their actiwity when the capacity of the sensory renal reflexes is too small to adjust larger disturbances in renal function.

Up to now, the prevalling view in this matter has been that neither the efferent nor the afferent renal nerves exert a tonic regulatory effect on renal excretory function under normal conditions. Studies performed on unanesthetized animals have generally demonstrated little or no difference between the function of a completely denervated kidney and the function of the contralateral innervated kidney. Furthermore, it has been shown that conscious unrestrained animals with completely bilaterally denervated kidneys are perfectly capable of maintaining normal sodium balance (Bencsáth et al., 1982). Clinical experience with renal transplantation, which necessarily involves complete renal. denervation, supports these results. However. it should be noted that the interpretation of these studies is hampered by the fact that when the renal nerves are removed, compensatory mechanisms may become activated in order to maintain sodium balance. After complete renal denervation changes in blood pressure, changes in plasma hormones or the development of denervation supersenstivity, may alter renal. function and mask the importance of the nerves in the control of sodium balance (Kline and Mercer, 1983; Stella et al., 1984; Krayachich et al., 1987).

An alternative way to study the potential role of renal nerves is to challenge the kidney to alter its excretory function. In recent experiments in consclous unrestrained rats, DiBona and Sawin (1983) showed that after bilateral denerwation rats were not able to maintain sodium balance during dietary sodium restriction. These results are supported by preliminary observations in conscious dogs by Schneider et al. (1978). However, in other studiles Bencsáth et al. (1982), Fernández-Repollet et al. (1985) and Mizelle et al. (1987) in consclous rats and dogs found that the renal nerves are also not essential for adaptation to chronic reductions in sodium intake. Despite the fact that these studies do not allow conclusions on involvement of one or the other nerve population in maintenance of sodium balance during dietary sodium restriction, it is generally thought that increased effecent renal nerve activity facilitates sodium reabsorp- 
tion under these circumstances (Bencsath et al., 1985; DiBona and Sabin, 1985; Gottschalk et al., 1985; 0sborn et al., 1987).

The purpose of the present study was to examine whether the sensory renal nerves contribute to the regulation of renal excretory function during normal and low sodium intake. We hypothetized that rats which were subjected to renal deafferentation, in contrast to intact rats, would show a diminished modulatory control upon efferent renal nerve activity and consequently renal excretory function when challenged with dietary sodium restriction. Since the efferent renal nerwes remain intact in the renal deafferented rats, it is, as discussed above, most likely that they wil come into balance during the low sodium intake. However, intact rats may reach this balance within a different time course than ARN-x rats do. To test for such transient differences, sham operated and renal deafferented rats were fed a sodium deficient diet supplemented with a continuous infusion of a Ringer"s solution. Rats received no other fluids. After the rats were in balance with this intake, the Ringer"s solution was exchanged for a modified Ringer's solution in which the sodium ion was replaced by the organic choline ion. The results $\mathbb{1}$. Ticate that $A R N-x$ rats initially loose more sodium than intact rats during the period of low sodium intake.

\subsection{Experimental protocol}

Uninephrectomized renal deafferented $(n=8)$ and sham operated rats $(\mathrm{n}=8)$ were kept in sodium and water balance by a constant intravenous infusion of a Ringer's solution (30 ml/24 hr). Only sodium depleted food was allowed. Experimental details on surgery, solutions and food are dem scribed in chapter 2. After $48 \mathrm{hr}$, the Ringer"s infusion was replaced by a similar solution containing $154 \mathrm{mM}$ choline chloride instead of 154 mM NaCl. Urine collections were made during periods of $90 \mathrm{~min}$, starting $24 \mathrm{hr}$ before until $24 \mathrm{hr}$ after the exchange of the solutions. Measurement of urlnary sodium, potassium and osmolality were made as described in section 2.6. At the beginning as well as at the end of the $48 \mathrm{hr}$ measuring perlod, resting mean arterial pressure (MAP) and heart rate (HR) were recorded. Arterial blood samples $(0.4 \mathrm{ml})$ were drawn for plasma renin concentration measurements. Furthermore, at the end of the choline-Cl infusion, MAP and HR responses to ganglionic blockade (hexamethonium, $20 \mathrm{mg} / \mathrm{kg}$ ) were determined. Possible parasympathetic influences were eliminated by $0.5 \mathrm{mg} / \mathrm{kg} \quad 1 . v$. atropine injection, 10 min before hexamethonium was given. Statistical. 
evaluation of differences between the period of normal and low sodium intake were analyzed by 2 -way analysis of variance. A Student's t-test for unpalred observations was used to test differences in urine data in the first 90 min after the start of the choline-cl infusions.

\subsection{Results}

Table 6.1 contains the hemodynamic as well as the urine data (cumulative over $24 \mathrm{hr}$ ) for the renal deafferented and sham operated rats during the period of normal sodfum intake and low sodium intake. During both periods, MAP and HR were not different in ARN-* and control rats. In the sham operated rats, average recovery of vater, expressed as percentage of fluid infused, was $5446 \%$. Recovery of sodium vas greater, $70_{ \pm} 5 \%$. These values represent the means over the $24 \mathrm{hr}$ period of normal sodium intake. During this period, cumulative urine output, sodium and potassium excretion were not different for the $\mathrm{ARN}-x$ and sham operated rats. Absolute concentrations of sodium and potassium in the urine vere $0.200 \pm 0.006$ and $0.091 \pm 0.003$ $\mu \mathrm{Eg} / \mu \mathrm{l}$ in the intact rats respectively. ARN-x rats exhibited similar values; sodium $0.009 \pm 0.007$ and potassium $0.092 \pm 0.004 \mu \mathrm{Eg} / \mu \mathrm{l}$. Urine osmolality was $1950 \pm 90$ and $1910 \pm 40$ mosm $/ \mathrm{kg} \mathrm{H} \mathrm{H}_{2} \mathrm{O}$ in the sham and ARN-x rats respective$1 y$.

The exchange of the Ringer's infusion for the choline-Cl infusion resulted in a similar significant rise $(p<0.01)$ in urine output in both the ARN-X and sham operated rats. This increased level was maintained as long as the choline infusion was continued. In the sham operated rats, urinary sodium concentration fell, as expected, gradually during this period. In the ARN-x rats, however, urinary sodium concentration was higher $(0.222 \pm$ $0.020 \mu \mathrm{Eg} / \mu 1)$ than in the control rats $(0.161 \pm 0.022 \mu \mathrm{Eg} / \mu 1)$ in the first 90 min perlod of the urine collections after the exchange of the solutions ( $t$-test: $p=0.056)$. Consequently, in comparison with the intact rats sodium excretion was significantly ( $<<0.05$ ) enhanced in the ARN- $x$ group. However, after this first collection period, similar values as in the control rats Were measured reaching ultimate values of less than 2 uEq/90 min, 24 hr after the start of the choline infusion. The results are show in detail in fig. 6.1. During the choline-Cl infusion, average urinary potassium concentrations fell to $0.063 \pm 0.003$ and $0.059 \pm 0.005 \mu \mathrm{Eg} / \mu \mathrm{l}$ in sham and $\mathrm{ARN}-\mathrm{x}$ rats respectively and were at no point in time significantly different between the groups. Also potassiun excretion was not significantly altered in both 
Table 6.1. Values of hemodynamic and urine data in renal deafferented (ARN-x) and sham operated rats during $24 \mathrm{hr}$ period of normal sadium intake ( $\mathrm{NaCl}$ ) and low sodium (choline-Cl $=$ ChCl) intake.

Significantly different from NaCl (ANOVA, p $<0.01$ ).

Sham

$(n=6-8)$
ARN-X

$(n=6-8)$
Body weight (grams)

start of experiment

end of experiment

Food intake

(grams)

MAP

( $\mathrm{mmH} \mathrm{H}$ )

HR

(beats/min)

Urine output

( $m l / 24 \mathrm{hr}$ )

Urine $\mathrm{Na}^{*}$ concentration

$(\mu \mathrm{Eg} / \mu \mathrm{l})$

Urine $\mathbb{K}^{+}$concentration

$(\mu \mathrm{Eg} / \mu \mathrm{l})$

Sodium excretion

$(\mu \mathrm{Eq} / 24 \mathrm{hr})$

Potassium excretion

( $\mu \mathrm{Eq} / 24 \mathrm{hr}$ )

Urine osmolality

$\left(\mathrm{mosm} / \mathrm{kg} \mathrm{H} \mathrm{H}_{2} 0\right)$

Plasma renin concen-

tration (ng $A \mathbb{I} / m l$.hr)

Response to hexame thonium

MAP (mmig)

HR (beats/min)
$323 \pm 14$

$309 \pm 14$

$15.9 \pm 1.5$ *

$11.7 \pm 2.0^{*}$

$108 \pm 2$

$106 \pm 2$

$345 \pm 7$

$346 \pm 9$

$16.1 \pm 1.7$ *

$24.4 \pm 2.5^{*}$

ChCl

$\mathrm{NaCl}$

ChCl.

$0.200 \pm 0.006$

not relevant

$\mathrm{NaCl}$

ChCl

$0.091 \pm 0.003$

$0.063 \pm 0.003^{*}$

$\mathrm{NaCl}$

$\mathrm{ChCl}$

$3302 \pm 206$
$1652 \pm 117$

$\mathrm{NaCl}$

$\mathrm{ChCl}$

$\mathrm{NaCl}$

ChCl.

$\mathrm{NaCl}$

Chel
$1473 \pm 81$

$1448 \pm 84$

$1950 \pm 90 *$

$1400 \pm 55^{*}$

$1.03 \pm 0.19$

$1.48 \pm 0.17$
$306 \pm 11$

$292 \pm 11$

$13.7 \pm 1.7$

$10.5 \pm 1.6$

$108 \pm 3$

$108 \pm 3$

$346 \pm 11$

$351 \pm 5$

$15.7 \pm 1.0 *$

$25.2 \pm 2.4^{\star}$

$0.209+0.007$

not relevant

$0.092 \pm 0.004 *$

$0.059 \pm 0.005^{\star}$

$3086 \pm 199$ *

$1973 \pm 166^{\star}$

$1354 \pm 171$

$1378 \pm 143$

$1910 \pm 40 *$

$1364 \pm 35^{\star}$

$0.89+0.16$

$1.09 \pm 0.19$
$-42 \pm 5$
$-43 \pm 4$
$-126 \pm 8$
$-118 \pm 9$

groups. Uxine osmolality decreased in the ARN- $x$ and control rats to $1364 \pm 35$ and $1400 \pm 55 \mathrm{mosm} / \mathrm{kg} \mathrm{H} \mathrm{H}_{2} 0$ respectively.

Plasma renin concentrations vere not different in ARN $x$ and control. rats during the period of nomal sodium intake. They were slightly increased after the choline infusion, but the differences were not statisti - 


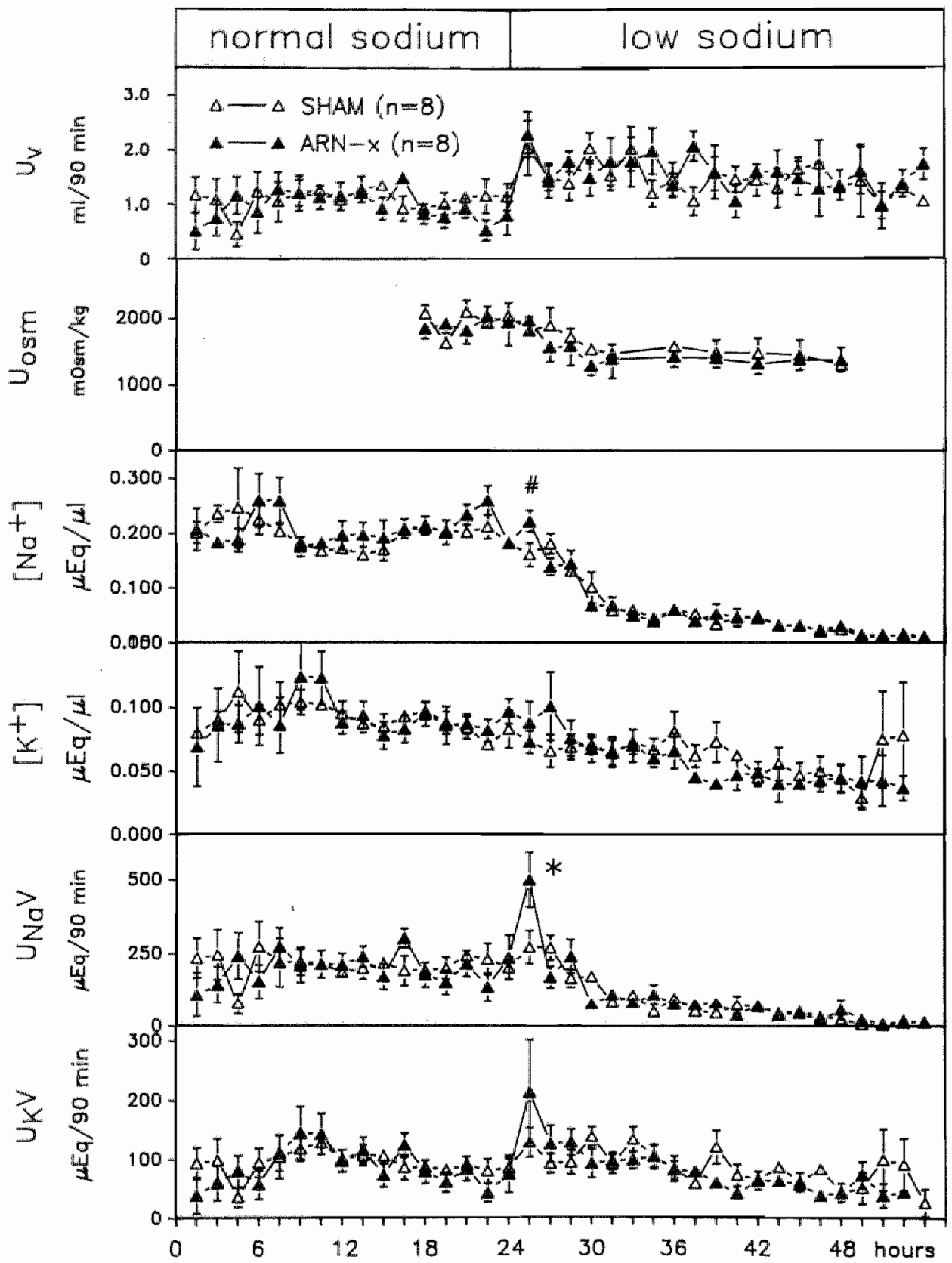

Fig. 6.1 Urine data in renald deafferented (ARN-x) and sham operated rats during a continuous ( $30 \mathrm{ml} / 2,4 \mathrm{hx}$ ) iv infusion of a Ringer's solution (normal sodium) anc choline-cl infusion (low sodium). Significantly different from sham: * $p<0.05$; \#p=0.06. 
cally significant (see table 6.1). Responses to ganglionic blockade with hexamethonium caused similar hemodynamic effects in the ARN-X and sham operated rats at the end of choline infusions.

\subsection{Discussion}

The goal of the present study was to examine whether ARN play a role in the control of renal excretory function during the transition from a normal to a low sodium intake. During the period of normal sodium intake, renal excretory function and hemodynamic parameters were similar in ARNand sham operated rats. This indicates that ARN are not essential in the control of water and sodium balance under these circumstances. When sodium intake was abruptly reduced by exchanging the infusion of the Ringer"s solution for the choline solution, there was a transient significant difference in sodium output between the ARN $\mathrm{x}$ and intact rats. For reasons discussed below, the switch to choline infusions increased urine flow rate in both groups of rats. This was accompanied by a surge in the excretion of sodium and to a lesser extent of potassium in the ARN-x but not in intact rats. If these differences between the groups resulted from a deficit in the contribution of ARN to the control of electrolyte excretion, the reason must have been that the ARN-x rats were unable to detect the elevated sodium and potassium excretion and to rectify it. Thus, in the intact rats ARN most likely have altered their activity upon the increase in urine sodium concentration and increased urine flow rate. These changes may have stimulated renal chemo- and mechanoreceptor activity respectively. Furthermore, considering that urine potassium concentration in combination with the intrapelvic pressure are most influential in the establishment of afferent activity levels (Moss, 1985, 1987) it can be reasoned that stince the urine potassium concentrations were not altered during the transition from saline to choline infustion, the increased urine flow rate may have excited renal $R_{2}$ receptors. In analogy to the backflow experiments with urine and isotonic potassium solutions (Recordati et al., 1978, 1980; Moss, 1987; chapter 3) the increased urine flow probably enhanced afferent act 1 wity since the increased intrapelvic pressure has not been offset by a concomftant decrease in urine potassium excretion. The increased ARA activity would on its turn increase ERN activity, resulting in an increased sodlum reabsorption (DiBona, 1982). Thus, the present data suggest that in intact rats ARN have a functional role in the early adjustments of renal excretory 
function to correct tramsient increases in sodium excretion. In contrast, ARN do not contribute to the necessary renal excretory changes when soidium restriction is prolonged. The present data show that during the continuaton of the choline-Cl infusion, $A R N-x$ rats reduced their sodium output to comparable levels as in intact rats, indicating that other more powerful and longer acting mechanisms have become activated.

A remarkable finding of this study was that in the ARN- $x$ as well as control rats during the whole perlod of choline-Cl infusion, the excretion of the amount of urine was significantly enhanced, when compared to the urine output during the Ringer infusion. Choline distribution and metabolism in the body is complex. Choline is taken up in relatively large amounts by the $\mathbb{1}$ ver and the kidney and is converted to betaine. Furthermore, it is incorporated into phospholipids throughout the body and it is converted into acetylcholine in nervous tissue (Sparf, 1973; Barlow and Marchbanks, 1985). The renal handling of choline in anesthetized rats has been examined by Acara et al. (1979). Their micropuncture experiments provide evidence for a progressive reabsorption of choline along the proximal conwoluted tubule, at low steady state plasma concentrations achieved by chollne infusion rates less than $7 \mu \mathrm{mol} / \mathrm{kg}$.min. At infusion rates of 14 umol/kg. min tubular reabsorption mechanisms for choline become saturated and choline is excreted in large amounts from the proximal tubule. Under these circumstances fractional excretion of choline is $50 \%$ higher than that of inulin, while GFR declines by approximately $20 \%$. Acara and co-workers further reported that choline may be reabsorbed in small amounts by the pars recta of Henle's loop, but that no further reabsorption of choline accurs along the more distal part of the nephron, which appears to be impermeable to choline. Since there is no further reabsorption of choline, the net secretion of choline consequently leads to an osmotically increased urine flow, as shown in the present as well as in Acara's study. Several other explanations for the Increased urine flow are possible. Whether the increased urine thow is caused by a shift of renal blood flow from cortical to medullary regions or by a decreased vasopressin release has not been determined. The fact that GFR declines (according to Acara, 1979) and that urine osmolarity in the first fractions after exchange of the solution does not differ from control walues makes these possibilities unlikely. The increased urine output could also be the result of changes in blood pressure. MAP and HR were, however, similar at the end of the period of Ringer and choline-Cl infuston. The decrease in sodium intake will finally result in a reduced filtered tubular sodium load. Since most of the sodium will be 
reabsorbed in the proximal tubules, sodium delivery to the loop of Henle and the distal tubules will probably be low and could be rate-ilmiting in the active sodium dependent reabsorptive processes (Hebert and Andreoli, 1984). This could lead to a reduced osmotic gradient in the kidney and hence an increased urine flow. It is, however, unlikely that this mechanism is responsible for the increased urine output during the first 90 min of the cholline infusion, since there 15 an abundance of $\mathrm{NaCl}$ in the extracellular fluid in these euvolemic animals. A final mechanism through which the choline infusion might have increased urine flow is an attenuation of the tubuloglomerular feedback mechanism. In the study of Acara and co-workers, GFR decreased by approximately $20 \%$ at comparable infusion rates of choline, as presently used. However, this reduction in filtration rate is evidently not enough to compensate for the extra volume loss. According to Thurau (1964) changes in the activity of the renin-angiotensin system may have been responsible for the decreased GFR. A remarkable finding in this respect is that in both the ARN-x and intact rats average plasma renin conm centration was not significantly altered 24 hours after the start of the sodium restriction. Low sodium chloride intake has been shown to increase plasma renin concentration several fold in experimental animals and humans (Davis and Freeman, 1976; Tucker and Blantz, 1987). Since chloride intake was kept constant during the whole experiment the present data are consistent with the theories and findings of Kotchen et al. (1987) that the amount of chloride rather than sodium transported at the macula densa determines renin release.

The above described arguments suggest that the increased urine flow is probably due to an osmotic diuresis, caused by a high renal secretion of choline, which is not compensated by an activation of the tubuloglomerular feed back mechanism. Whatever the mechanism of the increased urline output during the choline infusion, there are no indications that the processes underlying this phenomenon would be different in ARN $x$ and sharloperated rats. Therefore, taking into account that the bemodymanic data, urinary output and osmolality were similar in both groups of rats, the extra sodlum loss in ARN-x rats is most likely explained by an attenuated rellex regulation of efferent renal nerve activity. Since denervation natriuresis can be blocked by pharmacologlcal inhibition of prostaglandin synthesis (Barber et al., 1986) the early natriuresis in the ARN-x rats could also reflect a different renal prostaglandin metabolism in these animals. The present study was howewer not designed to test this hypothesis. It could further more be argued that the initial sodium excretion in the ARN-x rats is the 
result of a washout of sodiun accumulated in the ARt-x rats during the period of normal sodium intake. The fact that urine output and sodium and potassium excretion were comparable in both groups in the period before the choline infusion was started, makes this explanation unlikely. The present experiments are in favor of an ARN dependent neurogenic mechanism.

In anesthetized rats and cats ARN were shown to have an inhibitory influence on the contralateral efferent renal nerve activity (Kopp, 1985; Golin et al., 1987). The present experiments suggest an ipsilateral renorenal reflex and indicate that the ARN have an excitatory influence on the efferent renal nerves. Possible reasons for these discrepancies are numerous and may involve various neurogenic mechanisms which determine the balance of inhibitory and excitatory influences (Stella et al., 1987). A thorough analysis of these possibilities is beyond the scope of the present study. Tet, the existence of excitatory renorenal reflexes has been demonstrated by electrophysiological studies of Rogenes and in studies in dogs by Abildgaard al. (19B8), investigating the effects of renal venous pressure elevation on renal excretory function. The observation of non-uniform responses among the experimental studies may furthermore reflect the existence of a mixture of efferent renal nerve fibers which contact different targets in the kidney.

In conclusion, this is the first study in conscious unrestrained animals showing that afferent renal nerves play a modulatory role in the regulation of renal excretory function. They are not necessary in the maintenance of sodium balance but are able to correct transient increases in renal sodium excretion. Whether these sensory renal reflexes play a modulatory role in renal excretory function in humans is to be determined. 
7. ACUTE HEMODYNAMIC EFFECTS OF DIURETICS: ROLE FOR AFFERENT RENAL NERVES IN BODY-FLUTD VOLUME REGULATION?

\subsection{Introduction}

Diuretic drugs increase the renal excretion of solutes and water. They are amongst the most frequently used drugs in the treatment of hypertension and are prescribed on the empirical basis that they lower the systemic arterial blood pressure with a high degree of tolerability (Tay-. lor, 1985). The hemodynamic actions underlying their antihypertensive effect are, however, not fully understood.

The acute hemodynamic effects of diuretics differ from their longterm actions. Initially, diuretics cause a rapid fall in stroke volume and cardiac output. However, increased sympathetic nerve activity as a result of activation of carotid sinus, cardiopulmonary or intrarenal baroreceptors may oppose a rapid fall in blood pressure by increasing total peripheral resistance (Van Brummelen et al., 1980; Struyker-Boudier et al., 1983). In the long-term, TPR returns towards or even bellow control values. Several mechanisms, such as baroreflex adaptation, autoregulatory responses, de. creased sensitivity to various pressor stimuli or an enhanced production of endogeneous vasodilator substances have been implicated to contribute to these hypotensive processes (Davies and Wilson, 1975; Gerkens, 1987).

With respect to the acute effects, recent experiments from our labow ratory (Janssen et al., in preparation) have shown that surgical elimination of the baroreceptors blunts the increase in TPR after a bolus injection of chlorthalidone in conscious SHR. However, the same denervation procedure did not prevent the increase in TPR in response to furosemide. This indicates that these duretios may have differential acute cardiovascular actions.

The first purpose of the present study is to examine the hemodynamic mechanisms through which furosemide exerts its acute cardiovascular ef fects. It has been suggested by Ludens et al. $(1968,1969)$ that an increase in renal blood flow contributes to the diuretic effects of the drug. How ever, this would imply that furosemide induces a selective redistribution of cardiac output since TPR increases. To test this hypothesis, reglonal hemodynamic blood flow was monitored wh Doppler flowprobes in consclous rats after furosemide injections. Experiments vere performed under several 
conditions. To invesulgate the rolle of several hormonal systems in the rew sponse to furosemide (Vander and Carlson, 1969; Gerber, 1983), rats were pretreated with captopri1, indomethacin, or were infused with a RInger's solution to replete circulating volume. The second aim of this study was to investigate whether remal nervous factors are involved in the hemodynamic adaptations to diuretlc drug induced reductions of body fluid wolume. Since sino-aortic baroreceptors were not involved in the furosemide induced increase in TPR (see above), we hypothetize that the rise in TPR may be the consequence of an afferent renal nerve dependent sympathetic reflex activation. These renal sensors may be activated by the following mechanism. Furosemide inhibits the luminal. $\mathrm{Na}^{+}, \mathrm{K}^{+}, 2 \mathrm{Cl}^{-}$transport in the medullary thick ascending 1 imb of Henle. Consequently, it concentrates distal tubular Eluid and increases urine flow since less water is reabsorbed. The alterations in ionic composition of the renal tubular and pelvic fluids and the changes in intrarenal and intrapelvic pressures (Tucker and Blant $z, 1984$ ) may change the activity of chemo- and mechano-sensitive afferent renal nerves (cf. chapter 3 and 6 ). In turn, these nerves may elicit similar sympathetic reflexes as described in chapter 4 and reflexly increase TPR. To examine this hypothesis, experiments were performed in renal deafferented and sham operated rats. To study whether the effects of furosemide are the result of volume loss and not a direct effect of the drug, its efficacy was compared to those elicited by the carbonic anhydrase inhibitor acetazolamide. The contribution of efferent renal nerves to these processes was assessed in experiments on rats with a complete denervated kidney.

\subsection{Experimental protocols}

Male wistar rats weighing $280-340$ were implanted with Doppler flowprobes and catheters for administration of drugs and blood pressure mea surements as described in chaptex 2. To determine the amount of urine produced after injection of the diuretic drugs, rats were placed in diuresis cages (cf. section $2 m$ ). Changes in mean arterial pressure (MAP), heart rate (HR), reglonal blood flows and calculated vascular resistances vere measured up to 1 hour after injection of the diuretic drugs. The following experiments were performed (see also table 7.1):

1. In consclous rats, hemodynamic effects of bolus injections of furosemide (3 or $8 \mathrm{mg} / \mathrm{kg})$ and saline $(250 \mu 1)$ were examined. Experiments were performed in randomized order and a 48 hr interval was allowed before 
rats recelved another drug. To determine the role of several hormones in the hemodynamic response to furosemide, rats were pretreated ith captopril (30 $\mathrm{mg} / \mathrm{kg}$ ) or indomethacin ( $5 \mathrm{mg} / \mathrm{kg}$ ) $20 \mathrm{~min}$ before furosemide ( 8 $\mathrm{mg} / \mathrm{kg}$ ) was given. These doses are sufficient in blocking the anglotensin II and prostaglandin production respectively during the $1 \mathrm{hr}$ measuring period (Faber, 1987). Increases in MAP and renal resistance upon angiotensin I injection ( $75 \mathrm{ng} / \mathrm{kg}$ ) were significantly (ANONA $\mathrm{p}<0.01$ ) attenuated 5 min before injection of furosemide (data not shown). Hemodynamic parameters were also monitored during a concomitant iv infusion of a Ringer"s solution $(14 \mathrm{ml} / \mathrm{h})$, starting immediately after the injection of furosemide $(8 \mathrm{mg} / \mathrm{kg})$ to replete the circulating volume lost through diuresis. Experiments were done in one-kidney as well as two-kidney rats. Since the hemodynamic effects of the above mentioned drugs were comparable in both types of rats, pooled data are presented in the Results section.

2. In conscious one kidney rats, which were renally deafferented (ARN- $x$ ) or sham operated, hemodynamic effects of bolus injections of furosemide ( 8 $\mathrm{mg} / \mathrm{kg})$ and acetazolamide $(10 \mathrm{mg} / \mathrm{kg})$ were studied. Renal deafferentation was verified by comparison of the hemodynamic effects evoked by a 5 min intrarenal (ir) infusions of bradykinin (BK) at a rate of $1 \mu \mathrm{g} / \mathrm{min}$ in intact and $\mathrm{ARN}-\mathrm{x}$ rats.

3. In conscious rats with a completely denervated left and intact right kidney, renal hemodynamic changes were examined after furosemide ( $B$ $\mathrm{mg} / \mathrm{kg}$ ) injection. Rats were pretreated either with saline (control) or captopril ( $30 \mathrm{mg} / \mathrm{kg}$ ) $20 \mathrm{~min}$ before the diuretic was applied.

\section{$7.3 \quad$ Results}

For all the experiments described in this study base-line values of MAP and HR before injection of a diuretic drug and the urine production in 1 hour after infection are presented in table 7.1. The average MAP varled from $110 \pm 4$ to $124 \pm 5 \mathrm{mmHg}$. HR was between $329 \pm 21$ and $357 \pm 13$ beats/min. Urine production in 1 hour in non-diuretic (saline injected) rats was $0.4 \pm 0.2 \mathrm{ml}$. Furosemide injection at doses of 3 and $8 \mathrm{mg} / \mathrm{kg}$ increased urine production to $8.0 \pm 0.4$ and $13.7 \pm 0.6 \mathrm{ml}$ in 1 hour respectively. Acetazolamide raised urine production to $4.5 \pm 0.5 \mathrm{ml}$ in 1 hour.

A typical registration of the hemodynamic variables in response to furosemide is glven in fig. 7.1. As can be seen from the figure, renal 


\section{FUROSEMIDE}

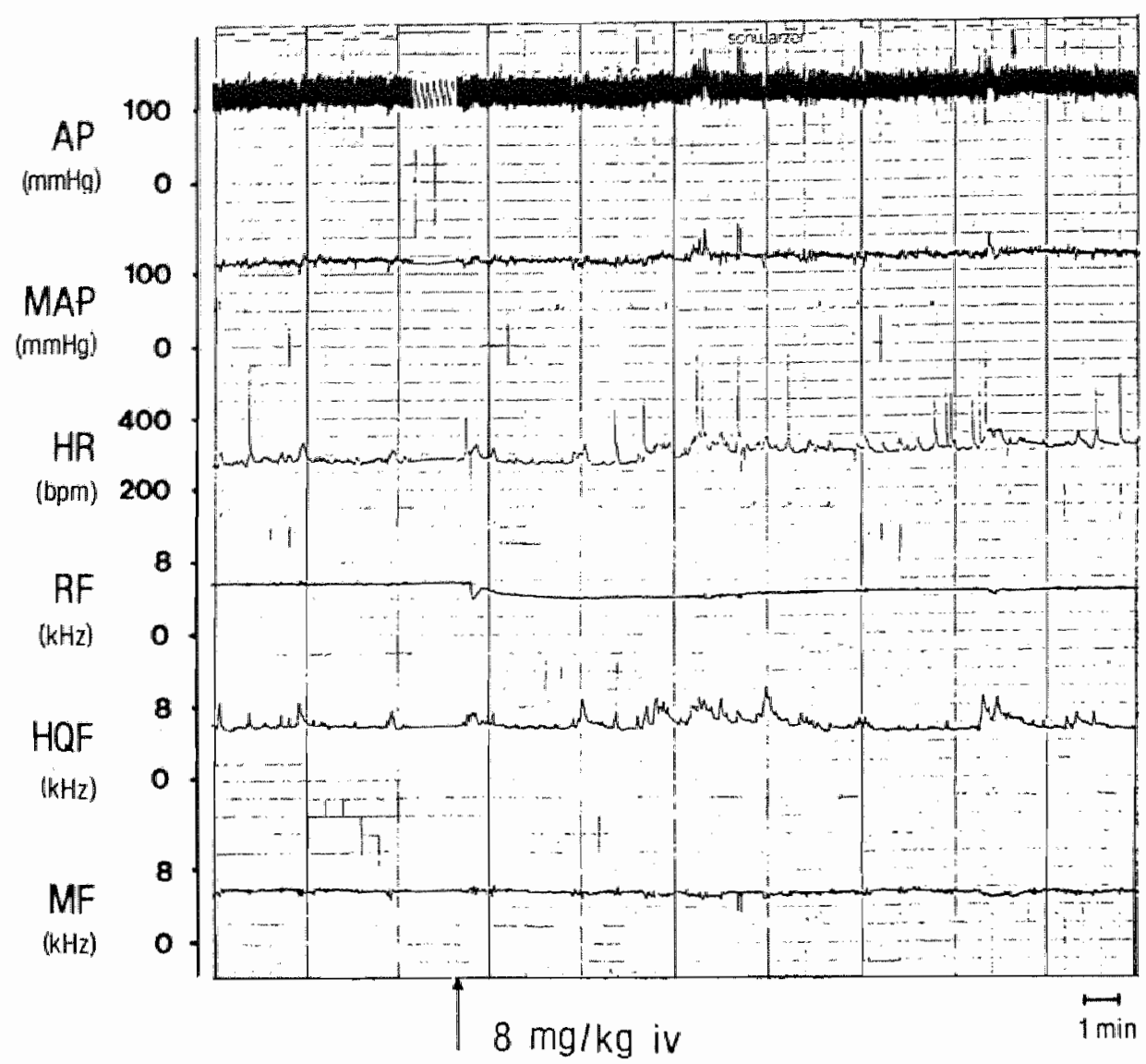

Fig. 7.1 Typical recording of regional hemodynamic effects after a bolus injection of furosemide in conscious istar rats.

blood flow decreased immediately by 10 -15\%, whout apparent changes in hindquater and mesenteric blood flow. The average hemodymamic effects of Iv injections of 3 and $8 \mathrm{mg} / \mathrm{kg}$ furosenide, compared to a $0.25 \mathrm{ml}$ saline control injection, are sumarized in fig. 7.2. During the 1 hour measuring period, changes in MAP and HR were not significantly different from values observed after saline injections. The early significant rise in renal resistance was followed by a gradual increase in resistance in the other vascular beds. Mesenterlc resistance rose maximally 15-20\%, 30 min after injection of furosemide. Hindquarter resistance was maximally increased 
Table 7.1. Hemodynamic baseline values before, and urine production in 1 hour after injection of diuretics. Walues are mean $\pm \mathrm{SE}$; $\mathrm{F}$ : furosemide; A: acetazolaride. \#aseline values after injection of captopril or indomethacin.

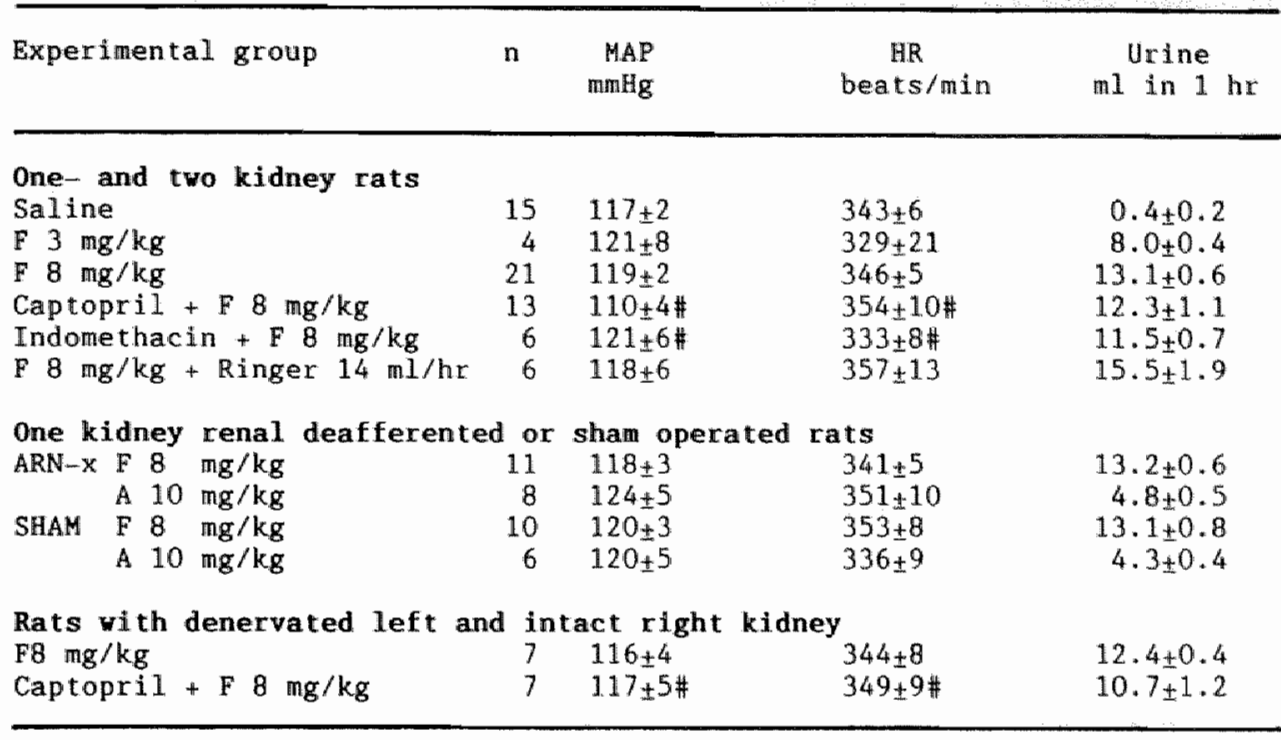

$30-40 \%$ at the end of the measuring perilod.

The effects of pretreatment with captopril and indomethacin on the furosemide induced vascular changes are presented in flg. 7.3. Before furosemide was injected, captopril or indomethacin administration itselves, had only minor hemodynamic effects. Captopril lowered MAP by $6 \pm 3$ mming and increased renal flow by $5 \pm 2 \%$. Indomethacin increased MAP by $6 \pm 3 \mathrm{~mm}$. w th a concomitant reduction in $\mathrm{HQF}$ by $7 \pm 4 \%$. The effects of these drugs on the other hemodynamic parameters were even less. As can be seen from fig. 7.3, pretreatment with captopr 11 had no effect on the early furosemide induced decrease in renal blood flow. However, during angiotensin I converting enzyme inhibition MAP started to fall $15 \mathrm{~min}$ after furosemide injection and this effect was associated with a significant inhibition of wasoconstriction in all vascular beds. In contrast to this effect of captopril, pretreatment with indomethacin did not attenuate or prevent any of the furosemide induced hemodynamic changes. The drug even slightly augmented the effects of furosemide. Fig. 7.3 also sumarizes the effect of wolume repletion on the furosemide induced vascular changes. Despite the infusion of 14 ml of a Ringer's solution furosemide caused similar effects as in nonvolume repleted aninals. 
The effects of furosemide were also studied in renal deafferented and intact sham operated rats. Renal deafferentation was werified by the absence of $1 \mathrm{H} B \mathrm{BK}$ induced afferent renal nerve dependent henodynamic reflex responses (see fig. 7.4). As depicted in fig. 7.5, the hemodynamic effects of a bolus injection of furosemide were similar in sham and ARN-x rats. Remarkably, in all rats acetazolamide caused a comparable increase in renal resistance as furosemide did. The effects on the hindquarter and mesenteric vascular bed were, however, significantly less pronounced than those induced by furosemide.

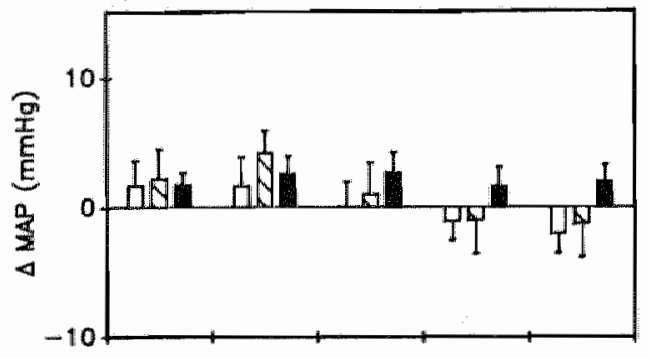

\section{$\square$ soline}

$\Delta S \mathrm{~g}$ furosemide $3 \mathrm{mg} / \mathrm{kg}$

furosemide $8 \mathrm{mg} / \mathrm{kg}$
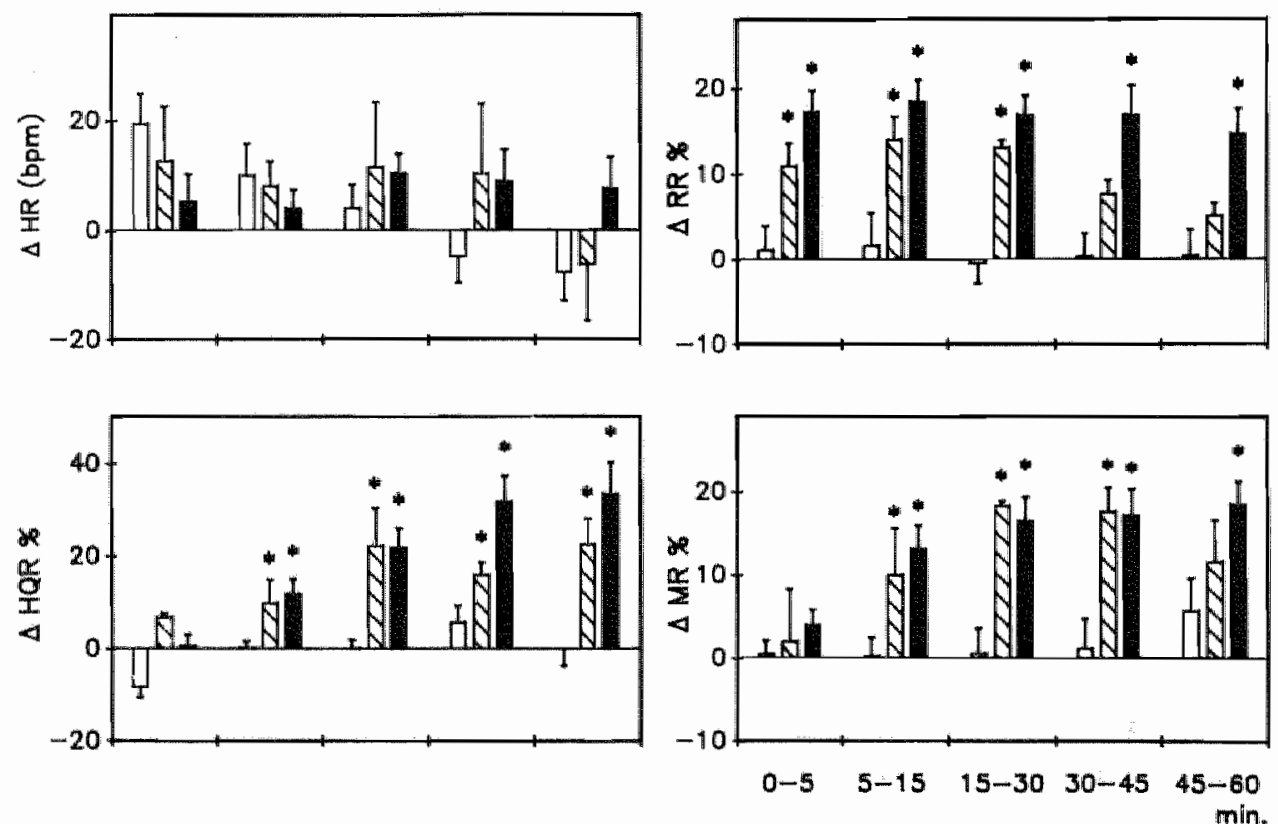

Fig. 7.2 Regional hemodynamic effects of bolus injections of furosemide in conscious rats.

Significantly different from saline: *p<0.001 (AMOVA) 
The effects of furasemide on renal hemodynamics were compared in rats with one completely denervated and one intact kidney. As sumarized in fig. 7.6, the furosemide induced decrease in renal blood flow was sinilar in the denervated and the intact kidney. Pretreatment with captopril did not prevent the early increase in resistance in both kidneys, but again reduced the later vasoconstrictive effects of furosemide.

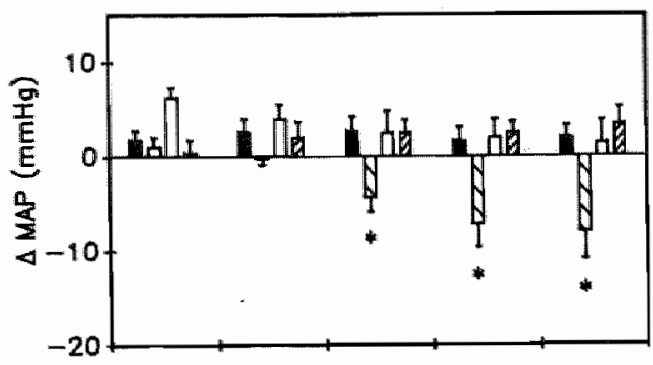

furosemide $8 \mathrm{mg} / \mathrm{kg}$ $\Delta$ captopril + F

$\square$ indomethacin $+F$

WII F + volume repletion
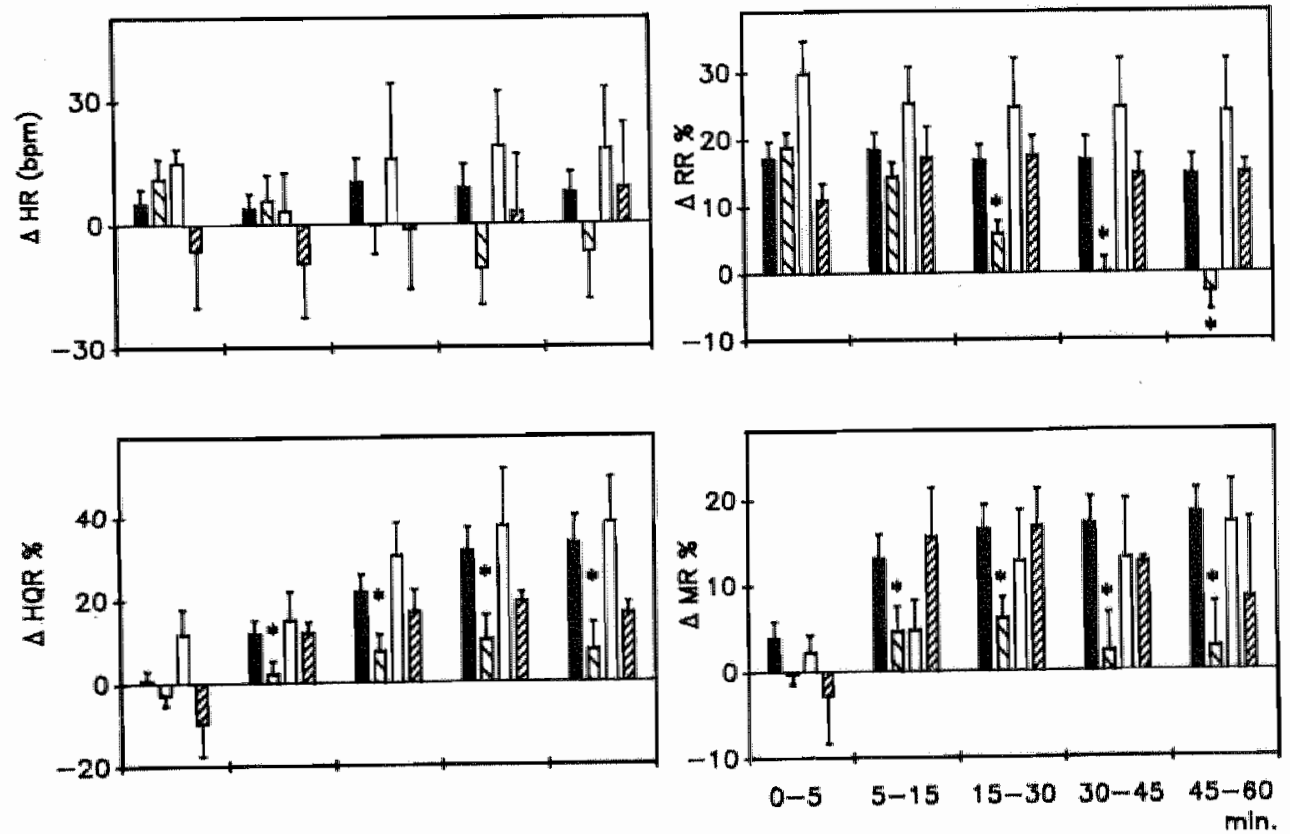

Fig. 7.3 Effects of pretreatment with captopril $(30 \mathrm{mg} / \mathrm{kg})$ and indomethacin ( $5 \mathrm{mg} / \mathrm{kg}$ ) on the regional hemodynamic changes induced by furosemide (F). Furthermore, the effects of volume repletion by an iv Ringer's infusion ( $14 \mathrm{ml} / \mathrm{hr}$ ) on the $\mathrm{F}$ induced changes are shown. Significantly different from furosemide: *p<0.05. 


\subsection{Discussion}

The purpose of this study was to evaluate the contribution of nervous and hormonal factors to hemodynamic mechanisms which underly the acute cardilovascular effects of furosemide. The resulls indicate that despite the reduction in cardiac output (cf. Struyker-Boudier et al., 19B3) and the huge diuresis - $13 \mathrm{ml}$ in 1 hour: this amount is similar to the blood volume of the rat - arterial pressure was maintained at a constant level. The regional flow measurements indicate that this was achieved by a redistribution of cardiac output, characterized by an acute but short lasting increase in renal vascular reststance, followed by a general AII dependent vasoconstriction in all vascular beds studied.

The mechanisms evoking the acute decrease in renal blood flow upon injection of furosemide are still unclear. This vasoconstrictor effect was

\section{Verification of ARN - x}

\section{Effects of intrarenal Bradykinin infusions}

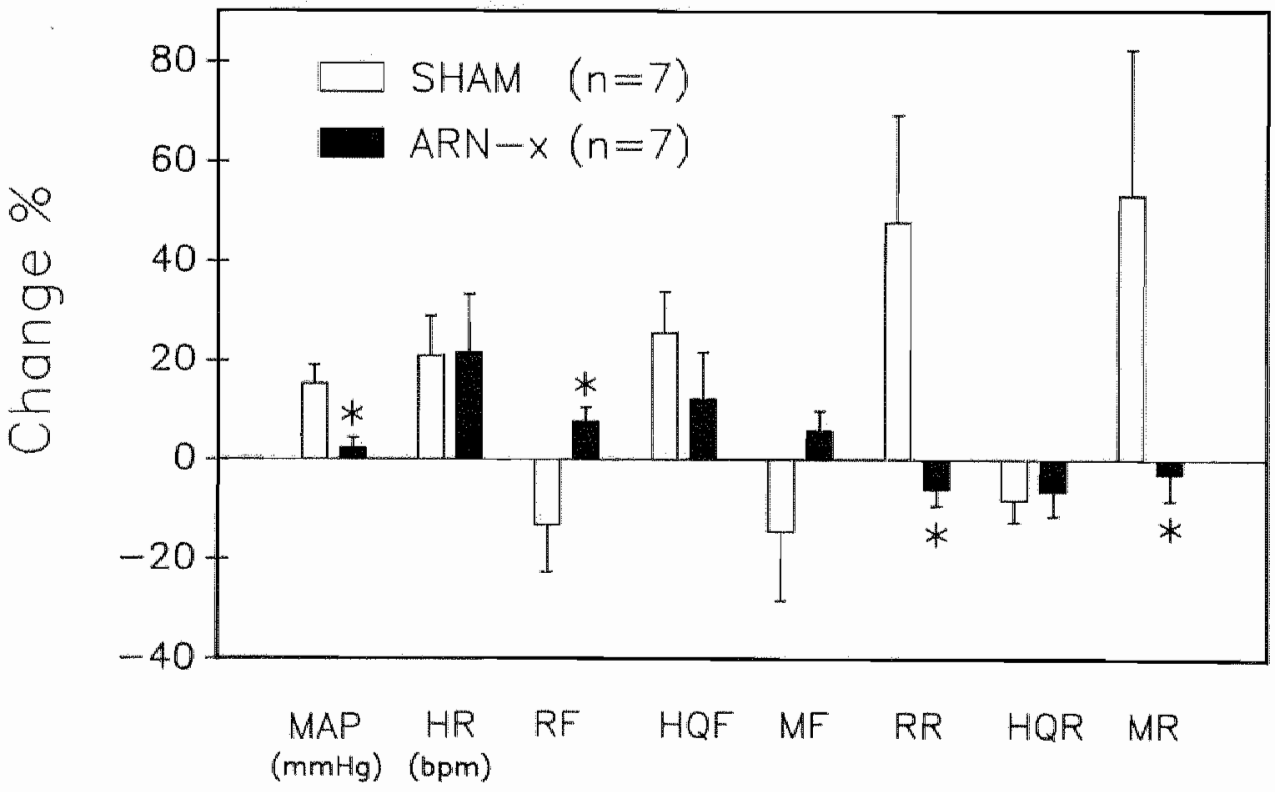

Fig. 7.4 Acute hemodynamic effects of ir BK infusions (1 $\mathrm{wg} / \mathrm{min}$ ) in renal deafferented $(A R N-x)$ and sham operated rats. Significantly different rom shan: * $\mathrm{p}<0.05$. 
not affected by volume repletion "inhibition of prostaglandin or angiotensin II production or elimination of the renal nerves. Preliminary experiments (data not shown) using theophyline suggested that this increase in renal resistance was not mediated through stimulation of adenosine receptors (cf. Osswald, 1984). Furthermore it is possible that the early renal vasoconstriction is the result of an elevated vasopressin release. The fact that during volume repletion similar effects were observed, however, argues against this hypothesis. Whether furosemide has a direct vasoconstrictor action on the renal resistance arteries is unknown. It has been shown by

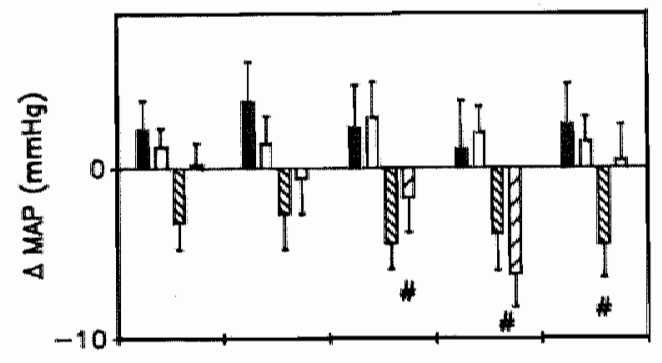

DHAM furosemide

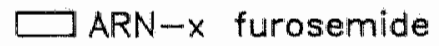

SHAM acetazolamide

ZZA ARN-x acetazolamide
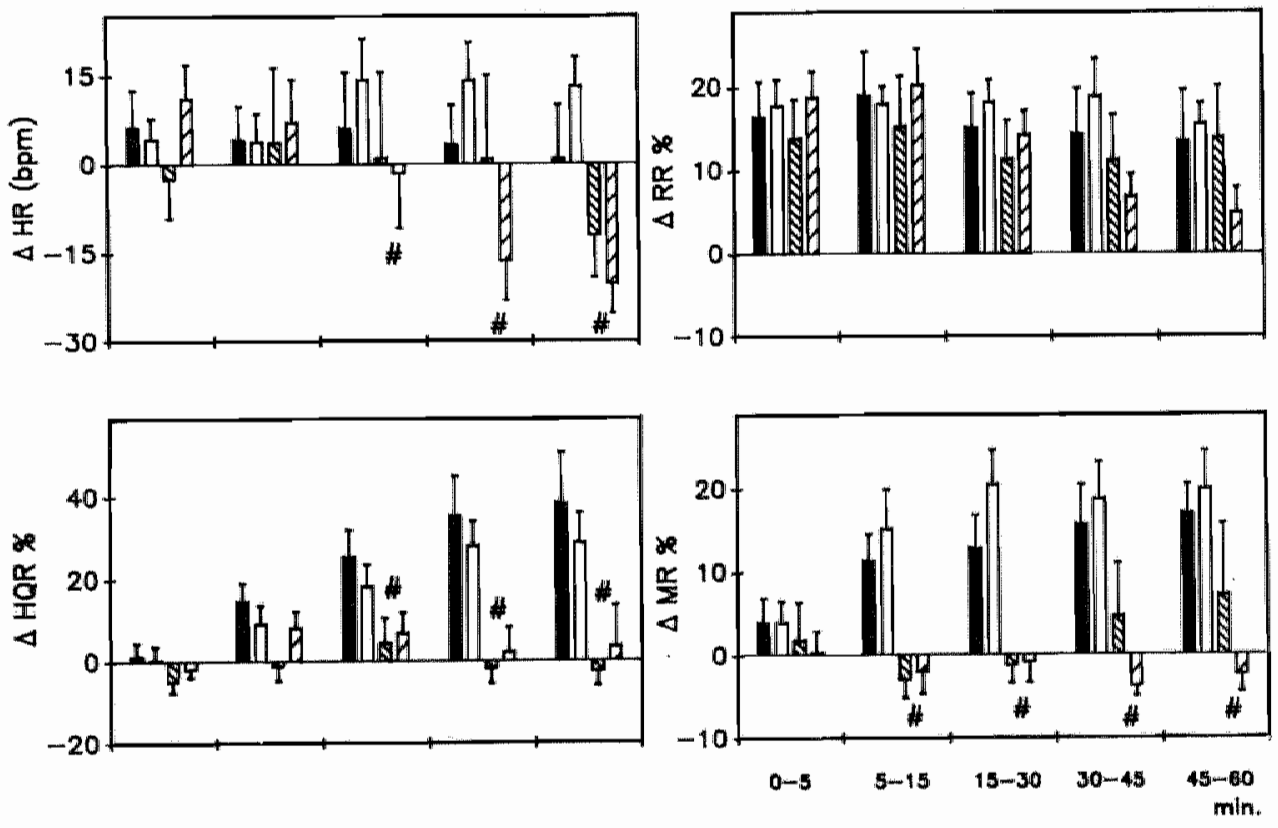

Fig. 7.5 Regional hemodynamic effects of furosemide ( $8 \mathrm{mg} / \mathrm{kg}$ ) and acetazolamide $(10 \mathrm{mg} / \mathrm{kg}$ ) in renal deafferented (ARN-x) and sham operated rats. Significantly different from furosemide: *p<0.05. 
Deth et al. (1987) that furosemide relaxes precontracted isolated rat aortic rings in a dose-dependent manner. Furthermore, Diksth t et al. (1973) repotted that furosemide increases venous compliance which suggests that, If anything, the drug has vasodilatory properties. Thus, up to now no pharmacological or physiological intervention had any effect on the furosemide induced renall vasoconstriction. This suggests that unknown intrinsic factors may be involved. A flrst explanation might be that the diuretic induced increase in renal resistance reflects the activation of the tubuloglomerular feedback mechanism. It has been observed repeatedly that administration of furosemide or acetazolamide results in reduction of Glomerular flltration rate (Burke and Duchin, 1979; Erik et al., 1982; Christensen et al., 1986), which is probably the result of preglomerular vasoconstriction. However, another report did not confirm this hypothesis and showed that

FUROSEMIDE

EX. CAPTOPRIL + F
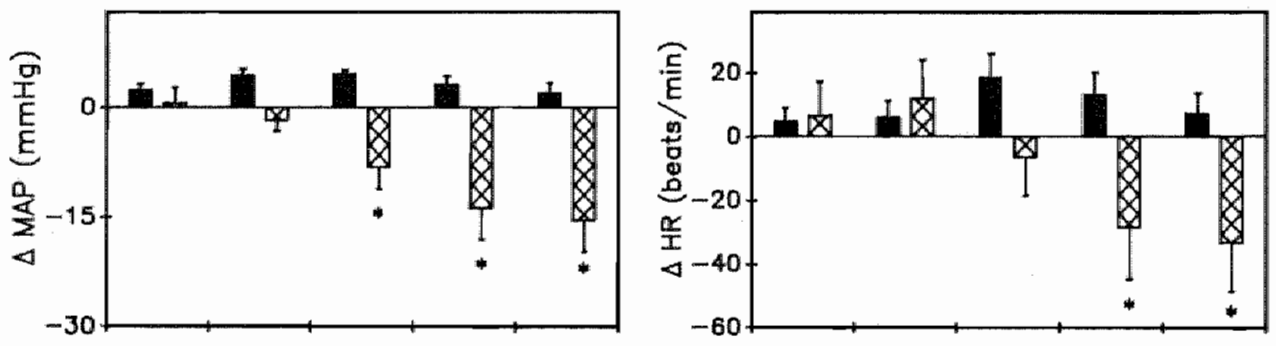

\section{FUROSEMIDE}

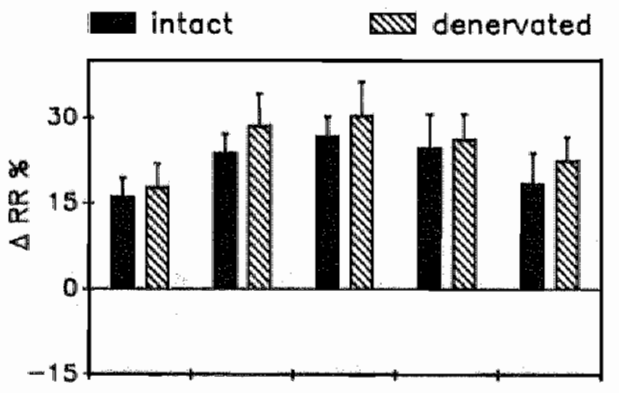

CAPTOPRLL + F

$\square \square$ intoct

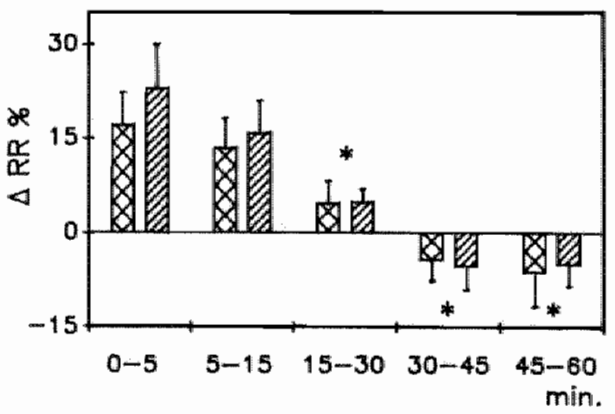

Fig. 7.6 Regional hemodynamic effects of furosemide $(8 \mathrm{mg} / \mathrm{kg})$ in rats with one intact kidney and one completely denervated kidney. Significantly different from control when pretreated with captopril: *p<0.05. 
tubuloglomerular feedback was attenuated upon furosemide administration and associated th a decrease in afferent arteriolar resistance (Tucker and Blantz, 1984). Furthermore, since the renin-angiotensin system is belleved to be the final mediator of the macula-densa-glomerulus feedback system, converting enzyme inhibition with captopril should have prevented the initial increase in renal resistance in the present experiments (Smits and struyker-Boudier, 1984). Altogether, this suggest that activation of the tubulo-glomerular feedback mechanism is not the rechanism for the early increase in renal resistance. Therefore, we propose a second mechanism, which partly depends on the tissue pressure theory, originally proposed by Hinshaw et al. (1959). Spitalewitz et al. (1982) reported that 10 min after furosemide injection $(3 \mathrm{mg} / \mathrm{kg}$ ) in dogs renal papillary plasma flow, measured by the albumin accumulation technique, was decreased by more than 50\%. Total renal blood flow, however, was slightly increased. These data suggest that following administration of furosemide, renal bllood flow is redistributed and shifted to the cortical layers. Comparable results for furosemide on medullary blood flow were obtained by Imbs et al. (1970). The reason for this redistribution of blood flow might be that, due to the furosemide induced reduction in papillary solute concentration, which approaches isotonicity, medullary vascular cells swell and thereby increase medullary vascular resistance. An indication for this mechanism might be the fact that renal interstitial hydrostatic pressure can rise by $7 \mathrm{~mm} \mathrm{Hg}$ during furosemide administration in rats (Tucker and Blantz, 1984). In this respect, the discrepancies in total renal blood flow following furosemide in rats (present study) and in dogs (Ludens et al., 1968; Spitalewitz et a1., 1982) might not reflect a different mechanism of action, but rather the anatomical and functional organization of the renal vasculature in both species. The greater the ratio of medullary and cortical blood flow in a species, the more a diuretic drug would increase total renal vascular resistance by this mechanism. As described by Ludens et al. (1969) furosenide in dogs also induced an initial 30-40\% transtent decrease in renal blood flow" This transient decrease might reflect the increase in renal medullary vascular resistance. Similar observations have been made in dogs when ureteral pressure was rapidly increased (Nash and selkurt, 1964). In dogs the diuretic induced acute vasoconstriction might be abolished by renal prostaglandin production. Williamson et al. (1975a, 1975b) showed that in this species, furasemide induces prostaglandin release to increase renall blood flow. Conversely, administration of inhibitors of prostaglandin synthesis lowers medullary plasma flow (Chuang et al., 1978) and renal 
blood flow (Gerber, 1983). In the present experiments, however, furosemide induced prostaglandin release is of minor importance since neither the acute nor the later hemodynamic effects were significanty influenced by pretreatment windomethacin.

The later generalized increase in resistance upon furosemide is, as indicated by the experiments wh captopril, dependent upon the activation of the renin angiotensin system. Also in humans, acute increases in peripheral vascular resistance hawe been measured when a bolus injection of furosemide was given (Johnston et al., 1986). Since these increases in peripheral vascular resistance were associated wh a rise in plasma renin activity, it was suggested that furosemide directly stimulates acute renin release in the kidney. The fact that in the present study even in volume repleted rats, mesenteric and hindquarter resistances were increased, supports this theory. Similar observations, that loop diuretics are potent stimuli for renin secretion even if sodium chloride and volume losses are replaced, were made by Vander and Carlson (1969). Injection of acetazolamide, however, did not elevate resistances in these vascular beds. In these experiments, blood pressure fell significantly, although diuresis was much less than in furosemide treated rats. As suggested by Kotchen et al. (1987), furosemide might interfere directly with the $\mathrm{Na}^{+}, \mathrm{K}^{+}, 2 \mathrm{Cl}^{-} \mathrm{co-}$ transport over the macula densa and thereby stimulate renin release direct$1 y$.

The fact that furosemide caused similar hemodynamic changes in the intact as well as in the completely denervated kidney, both in the absence and in the presence of captopril, indicates that furosemide does not cause a reflex activation of efferent renal nerves. Thus, the later furosemide induced wascular changes are not the results of a reflex activation of sympathetic efferent nerve activity. This supports also the finding that furasemide does not cause baroreceptor reflex activation (Janssen et al., in prepration).

The involvement of sensory renal nerves in the cardiovascular adaptatlons to diuretic induced volume loss was assessed by comparing the effects of furosemide and acetazolamide in selective renal deafferented and sham operated rats. The results indicate that there is no difference in hemodynamic reflex regulation between $A R N-x$ and sham operated rats, when elther one of the diuretics were injected. Thus, the so-called renal baroreceptors are not involved in the acute cardiovascular adaptations to loss of circulating volume induced by diuretics. Although, renal interstitial hydrostatic pressure may rise by $7 \mathrm{~mm}$ Hg (Tucker and Blantz, 1984), this increase 
may be too low to activate the renal mechanoreceptors. Cardiovascular reflex activation via stimulation of renal chemoreceptors was probably suppressed, due to the diuretic induced wash-out of the renal medullary gradient. Evidence from electrophysiological studies (cf. chapter 3) indicates that spontaneous as well as stimulated afferent renal nerve activity can be reduced by diluting the urine. However, as described in chapter 6 , sensory renal nerves may play a role in the modulation of renal sodiut excretion. In the present experiments, however, urine samples were not analyzed.

In sumary, the acute regional henodymamic effects of furosemide in conscious rats are characterized by an immediate increase in renal resistance followed by a gradual increase in resistances of other vasculat beds. The nature of the early renal vasoconstriction remains unclear, but possibly relates to changes in intrarenal pressure. The late generalized vasoconstriction was shown to be dependent upon activation of the renin angiotensin system. A role for renal sensors in the acute cardiovascular adaptations to diuretics could be excluded by selective renal deaferentation. 
8. EFFECTS OF COHPLETE RENAL DENERVATION AND SELECTIVE AFFERENT RENAL DENERVATION ON THE HYPERTENSION INDUCED BY INTRAREWAL NOREPINEPHRTNE TNPUSTON IN CONSCTOUS RATS

\subsection{Introduction}

Renal nerves contain both efferent and afferent fibers (Moss, 1982). Both nerve populations are considered to be involved in the pathogenesis of some forms of hypertension (Hoss, 1982; Katholi, 1982, 1985). Evidence for an important role of the efferent renal nerves comes from studies in which renal adrenergic hyperactivity was mimicked by infusions of norepinephrine directly into the renal artery. In conscious dogs this intervention causes sustained hypertension which is characterized by a positive sodium balance and fluid retention (Katholi et al. "1977; Cowley et al., 1979). Klelnjans et a1. (1984) showed that ir NE infusions in conscious rats produces sustained hypertension without fluid retention. During 5-day ir infusions of NE in conscious rats blood pressure was consistently increased above levels reached during iv infusions of the same amounts. The extra pressor response was not associated with changes in glomerular filtration rate, increased plasma sodium levels, or an elevated plasma volume (Kleinjans et al., $1984 \mathrm{~b}$ ). The involvement of the renin-angiotensin system was also excluded (Kleinjans, 1983).

One of the most striking observations by Kleinjans et al. (1984b) was that rats, infused ix ith $N E$, exhibited significantly higher plasma NE lem wels than iv infused rats, while no differences in NE clearance were observed (Kleinjans et al., 1983). This suggested that direct application of NE to the kidney causes an actiwation of the sympathetic nervous system. This mechanism could be mediated through activation of afferent renal nerves, similar to the NE-induced stimulation of carotid sinus baroreceptors afferents (Mills and Smith, 1983). Several authors have shown that stimulation of ARN by ir infusion of adenosine (Katholi et a1., 1983, 1985) or bradykinin (Smits and Brody, 1984) or, alternatively, through a reduction of renal blood flow (Faber and Brody, 1985), causes a sympathetically mediated increase in blood pressure which can even lead to sustained hypertension (Katholi et al., 1985).

The present study was designed to test the hypothesis that chronic ir NE infusions produce hypertension via activation of ARN. Two different 
approaches were chosen. In one experimental group, rats were subjected to a complete surgical section of both nerve populations before starting a 5 day continuous ir NE infusion. Because this procedure may induce supersensitivity to NE (Kline and Mercer, 1980), the fect of ir NE was also studied in group subjected to selective afferent renal denervation.

\subsection{Experimental protocols}

Male Wistar rats, aged 12-16 weeks, were used in this study. All rats underwent a left-sided nephrectomy at least 4 weeks before they underwent the surgery for renal denervations. During the whole experimental period, rats had free access to standard food and water. Rats were subjected to complete renal denervation (RN-x) or selective afferent renal denervation (ARN-x) or the appropriate sham procedures. After $7-8$ days of recovery, rats were instrumented with catheters for blood pressure measurements and ir and iv infusions. Surgical techniques are described in detail in chapter 2.

Before measurements were started, rats were allowed to recover for additional 2-3 days. In each experimental group MAP and HR were recorded on 2 control and 5 experimental days between 9 and 12 a.m., using standard procedures (see section 2.1).

The following experiments were performed:

1. Twenty-five $R N-x$ and 26 sham $R N-x$ rats received during the 2 -day control period an ir saline infusion using osmotic minipumps (flow rate: $1 \mu \mathrm{l} /$ hr). Then, under light ether anesthesia, saline pumps were replaced by pumps containing saline ( $R N-x: n=8$; sham $R N-x: n=9$ ) or $N E((-)$ norepinephrine bitatrate) in concentrations that achieved ir infusions of free

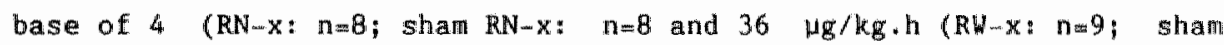
RU-x: $n=9$ ). Ascorbic acid ( $1 \mathrm{mg} / \mathrm{ml}$ ) was added to the solutions to prevent oxidation of $N E$ (see Kleinjans et al., 1981). From all rats, $0.5 \mathrm{~m}$ ] arterial blood samples vere drawn on control day 2 and experimental day 1 and 4 to measure plasma NE concentrations. The plasma samples were stared at $-80^{\circ} \mathrm{C}$ until assayed (cf. section 2.9.2).

2. Six $\mathrm{RN}-\mathrm{x}$ and 6 sham $\mathrm{RN}-\mathrm{x}$ rats received after 2 control days a 5-day 12 $\mu \mathrm{g} / \mathrm{kg} \cdot \mathrm{hr}$ ir infusion of NE. On control day 2 and experimental day 4 , a $0.4 \mathrm{ml}$ arterial blood sample was taken for measurement of plasma renin levels (cf. section 2.9.1).

3. Fourteen $A R N-x$ and 16 sham $A R N-x$ rats were studied. After the blood 
pressure measurements on the first control day, rats were briefly anesthetized with ether to disconnect the osmotic minipump from the ir catheter. Then the catheter was filled with heparinized saline (5 IU/ml) and exteriorlzed in the neck. After the blood pressure measurements on the second control day, BK was infused ir to verify ARN-X as described below in detail. Then under light ether anesthesia osmotic minipumps filled 1 th NR in amounts to release $12 \mu \mathrm{g} / \mathrm{kg} \cdot \mathrm{hr}$ were implanted and connected either to the ir or to the iv catheter for a 5-day continuous infusion. On control day 2 and experimental day 4 , a $0.9 \mathrm{ml}$ blood sample was taken for assessment of plasma NE and plasma renin lewels.

4. To study whether the surgical procedures had any influences on the blood pressure measurements, ARN-X and sham ARN-x rats were infused either ir or iv with saline (ARN-x: ir: $n=3$, iv: $n=3$; sham ARN-x: ir: $n=3$, iv: $\mathrm{n}=2$ ). Throughout the experimental period, all values for MAP and HR did not deviate significantly from control levels (table 8.1).

\section{Verification of the renal denervations}

1. RN-x: At the end of the experimental protocol, the osmotic minipumps were taken out under light ether anesthesia. The ir catehter was plugged to prevent leakage of $\mathrm{NE}$. The next day, rats were anesthetized with ether and the right kidney was rapidly excised, weighed and prepared for measurement of $\mathrm{NE}$ content as described in section 2.9.2.

2. ARN-x: Smits and Brody (1984) showed that ir infusions of bradykinin (BK) in conscious rats causes an immediate increase in MAP and HR through activation of afferent renal nerves. In pilot experiments we demonstrated that dorsall rhizotomy (T9-T13) abolishes the effects of ir BK infusions (Janssen et al., 1985).

Therefore, rats were given a $5-m i n$ ir and iv infusion of BK $(0.3$ and 1.0 $\mathrm{Hg} / \mathrm{min}$ of the $\mathrm{tri-acetate}$ salt (SIgma). Changes in MAP and HR were calculated using the pre-infusion values and the mean values of the last $3 \mathrm{~min}$ during $\mathrm{Bk}$ infusion.

At the end of the experiments, animals were perfused transcardially with 10\% formaline. The spinal cord was dissected and inspected under a dissection microscope. Only the animals in which a complete transsection of the dorsal roots T9-T13 was observed were included in the data as being deafferented. In the sham controls, all dorsal roots were intact.

- A11 data were statistically analyzed by means of a 2- or 3-way ANDVA and relevant $t$-statistics as indicated in section 2.10 . 
Tabile 8.1. MAP and HR on control and experimental days in renal deafferented $(A R N-x)$ or sham operated rats during saline infusions (data are pooled for intravenous and intrarenal infusions).

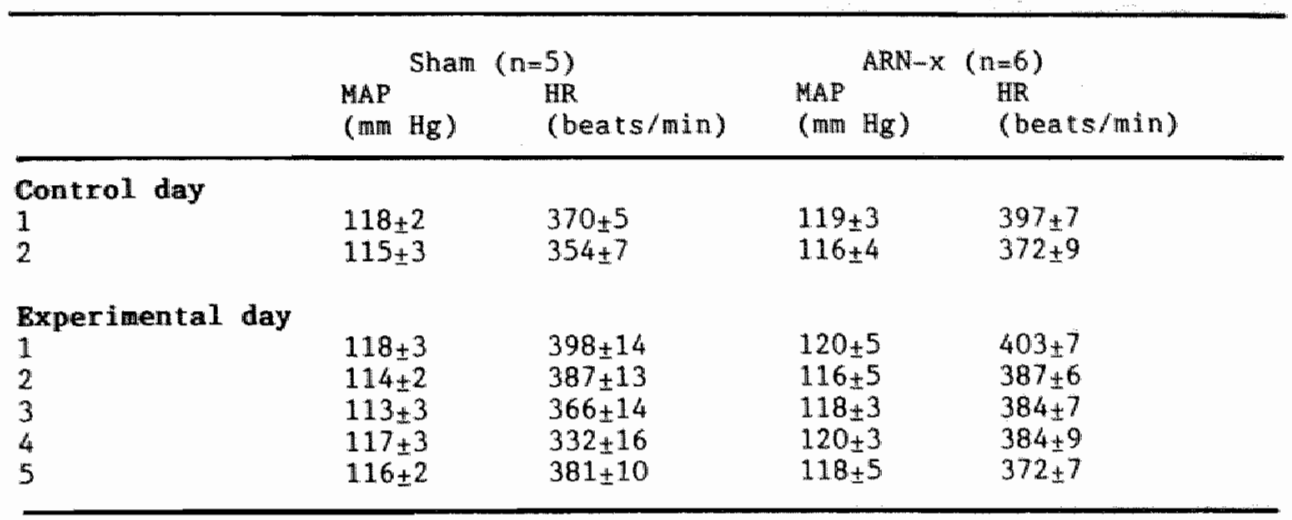

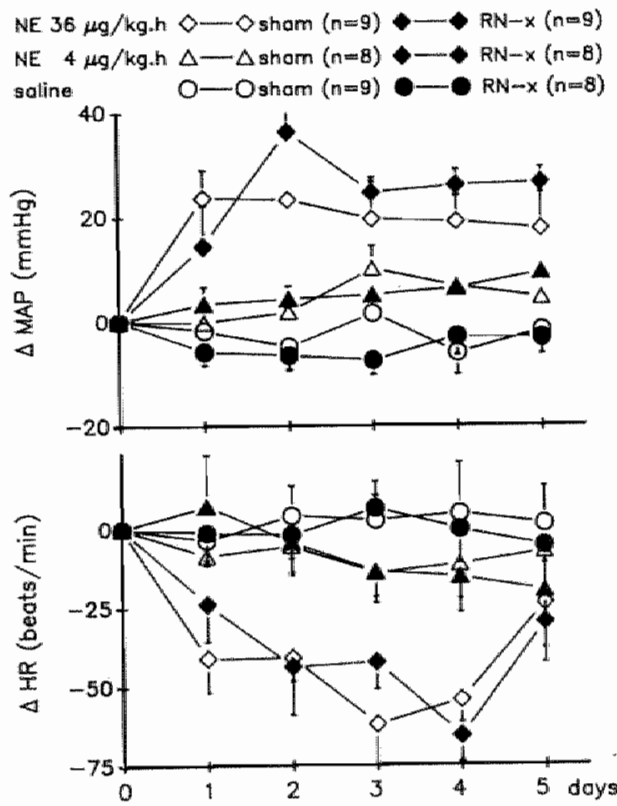

Fig. 8.1 Changes in MAP and HR in completely renal denerwated rats (RN-X) and sham operated rats during ir infusion of saline and NE. ANOVA revealed no statistical difference between sham operated and $\mathrm{RN}-\mathrm{X}$ ratis.

\subsection{Results}

8.3.1 Effects of chronic ir NE infusion in rats following total renal denervation

Total renal denervation reduced renal $N E$ content by $86 \%$ (RN-X $(n=22)$ 


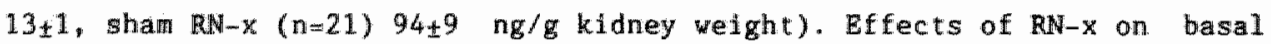
MAP and HR are described in table 8.2. MAP vas significantly (p<0.001) lower in RN-x than in sham RN-x rats. HR did not differ significantly. Fig. 8.1 summarizes the effects of ir infusion of $\mathbb{N E}^{\mathrm{in}} \mathrm{RN}-\mathrm{X}$ and sham $\mathrm{RN}-\mathrm{X}$ rats. In both groups, continuous it infusion of NE increased MAP significantly (p60.01) as compared to levels of MAP during ir saline infusion. MAP increased in sham $R M-x$ during it infusion of $4 \mu \mathrm{g} N \mathrm{NE} / \mathrm{kg}$.hr to $120 \pm 3 \mathrm{~mm} \mathrm{Hg}$ (mean value of 5 days of infusion). A 5 -day ix $\mathrm{NE}$ infusion of $36 \mathrm{\mu g} / \mathrm{kg} \cdot \mathrm{hr}$ brought MAP up to $134 \pm 5 \mathrm{~mm} \mathrm{Hg}$.

Blood pressure levels in $\mathrm{RN}-\mathrm{x}$ rats rose to $114 \pm 3$ and $134 \pm 5 \mathrm{~mm} \mathrm{Hg}$ durfing 4 and $36, \mu \mathrm{NE} / \mathrm{kg} . \mathrm{hr}$ infusions, respectively. ANOVA revealed no statistical differences between the response to ir NE infusions in intact and RN-X rats. HR decreased significantly as compared to control levels during 36, but not during $4 \mathrm{\mu g} \mathrm{NE} / \mathrm{kg} \cdot \mathrm{hr}$. No differences between the response of $\mathrm{RN}-\mathrm{X}$ and sham $\mathrm{RN}-\mathrm{X}$ were observed.

Table 8.2. MAP and HR on control days (mean of 2 days) in total renal denervated $(R N-x)$, renal deafferented $(A N R-x)$, and sham operated controls. * $\mathrm{p}<0.001$ compared to sham $\mathrm{RN}-\mathrm{x}$, Student's t-test.

\begin{tabular}{llll} 
Group & $\mathrm{n}$ & MAP $(\mathrm{mm} \mathrm{Hg})$ & HR (beats/min) \\
\hline Sham RN-x & 26 & $117 \pm 1$ & $382 \pm 5$ \\
RN $-x$ & 25 & $109 \pm 1 *$ & $376 \pm 5$ \\
Sham ARN-x & 16 & $118 \pm 2$ & $356 \pm 5$ \\
ARN $-x$ & 14 & $118 \pm 2$ & $366 \pm 6$
\end{tabular}

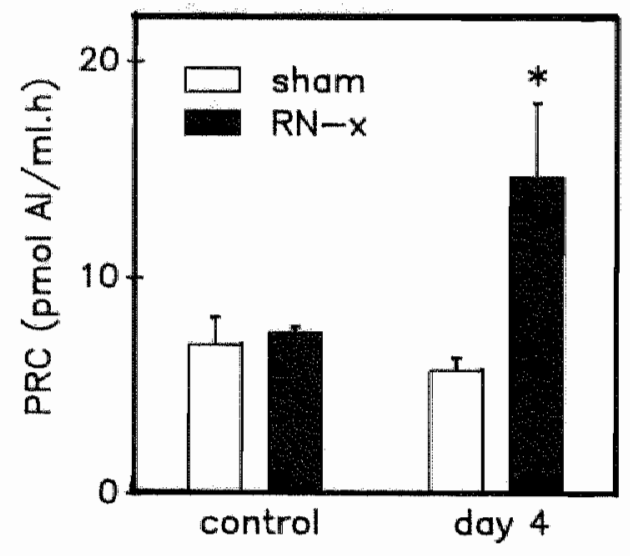

Fig. 8.2

Effects of ir NE (12 $\mathrm{Mg} / \mathrm{kg} \cdot \mathrm{hr})$ infusions on plasma renin concentram tion (PRC) in sham operated $(n=6)$ and completely renal denervated ( $\mathrm{RN}-\mathrm{x}$ ) rats $(\mathrm{n}=6)$ on control day and day 4 of infusion. Significantly different from sham: *p< 0.05 . 
In table 8.3 the effects of ir NE infusion on plasma NE levels are shown. Plasma NE concentrations on control days were not significantly different in RN-X and sham $R N-x$ rats. During ir infusion of $4 \mu \mathrm{g}$ NE/kg.he plasma NE concentration rose slightly in RN-X rats, but this increase was similar to that in intact rats. Thirty-six $\mu \mathrm{g} N \mathrm{NE} / \mathrm{kg} \cdot \mathrm{hr}$ ir infusion raised plasma NE concentration in both groups to comparably high levels.

In a separate group of sham $\mathrm{RN}-\mathrm{x}$ and $\mathrm{RN}-\mathrm{x}$ rats, $12 \mu \mathrm{g} \mathrm{NE} / \mathrm{kgg}$.hr was infused ir for 5 days to study effects on PRC. In RN-X rats, MAP rose froin $108 \pm 3$ (control) to $137 \pm 3 \mathrm{~mm} \mathrm{Hg}$ on experimental day 4 . In sham $\mathrm{RN}-\mathrm{x}$ ir $\mathrm{NE}$ infusion increased MAP from $119 \pm 2$ to $139 \pm 6 \mathrm{~mm}$ fig, respectively. During ir NE in $\mathrm{RN}-\mathrm{x}$ rats plasma renin concentration (see fig. 8.2) was significantly ( $p<0.05)$ raised from $7.5 \pm 0.3$ to $14.7 \pm 3.5 \mathrm{pmol} A I / \mathrm{ml} . \mathrm{hr}(\mathrm{p}<0.05)$ as conpared to PRC levels during ir NE infusion in intact rats (from 6.9 1.3 to $5.7 \pm 0.6$ pmol AI/mI.hr).

Table 8.3. Plasma $\mathrm{NE}$ concentrations in total renal denervated ( $\mathrm{RN}-\mathrm{x}$ ) or sham operated rats on control day and day 1 and 4 of intrarenal infusion of saline and 4 or $36 \mu \mathrm{g} \mathrm{NE} / \mathrm{kg} . \mathrm{h}$. 3-way ANOWA (RN-x vs. shan): NS.

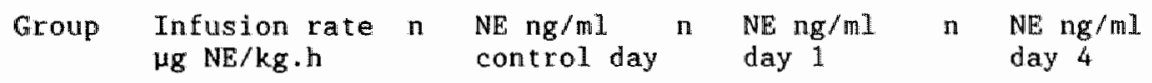

$\begin{array}{llllllll}\text { Sham } & \text { saline } & 7 & 0.59 \pm 0.22 & 9 & 0.27 \pm 0.06 & 7 & 0.35 \pm 0.07 \\ \mathrm{RN}-\mathrm{x} & \text { saline } & 6 & 0.61 \pm 0.22 & 8 & 0.31 \pm 0.07 & 7 & 0.25 \pm 0.03 \\ \text { Sham } & 4 & 7 & 0.39 \pm 0.16 & 6 & 0.62 \pm 0.14 & 8 & 0.57 \pm 0.08 \\ \mathrm{RN}-\mathrm{x} & 4 & 5 & 0.45 \pm 0.12 & 4 & 1.37 \pm 0.91 & 6 & 0.88 \pm 0.16 \\ \mathrm{Sham} & 36 & 7 & 0.33 \pm 0.08 & 7 & 2.12 \pm 0.32 & 7 & 3.81 \pm 0.67 \\ \mathrm{RN}-\mathrm{x} & 36 & 6 & 0.57 \pm 0.19 & 8 & 2.81 \pm 0.60 & 7 & 3.73 \pm 0.91\end{array}$

\subsubsection{Effects of chronic ir and iv NE infusion in renal deafferented rats}

Verification of renal deafferentation was performed by comparing the effects of iv and ir BK infusion on MAP and HR in intact and ARN-x rats. The results are presented in fig. 8.3. During ir Bk infusion of 0.3 and 1.0 $\mu \mathrm{g} / \mathrm{min}$, MAP rose 10 and $19 \mathrm{~mm} \mathrm{Hg}$, respectiwely, in sham ARN-x rats. This response was significantly different ( $p<0.001$ ) from values obtained during iv infusions of the same amounts ( 1 and $5 \mathrm{~mm} / \mathrm{Hg}$, respectively). In ARNrats both infusion rates of BK failed to increase MAP significantly when infused ir. The response in ARM- $x$ rats was not significantly different as compared to control levels during iv infusion (ir: 2 and 9 mor $\mathrm{Hg} ; \mathrm{iv:} 2$ and 
8. Ham Hg), suggesting functional afferent renal denervation. The effects on IR during. BK infusions in ARN- $x$ and sham ARN-x rats showed a similar pattern as described for MAP although the differences between groups are less pronounced. The effect of 115 BK on HR in ARN-x rats was neither significantly different from the effects observed in intact rats, nor different from the results of iv BK infusion in ARM-x rats (see fig. 8.3).

BRADYKININ $0.3 \mu \mathrm{g} / \mathrm{min}$
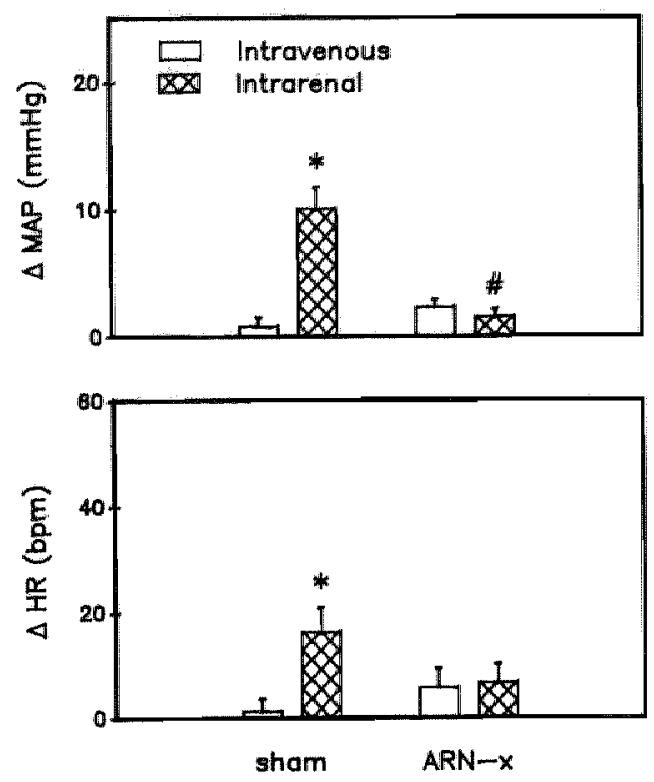

BRADYKININ $1.0 \mu \mathrm{g} / \mathrm{min}$
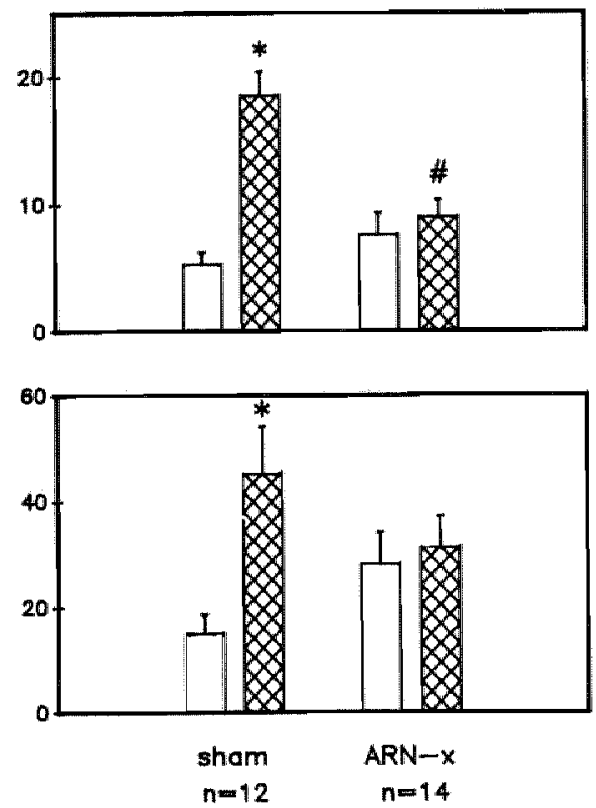

Fig. 8.3 Changes in MAP and HR during iv and ir infusions of BR in renal deafferented (ARN-X) and sham operated rats. Significantly different between ir and $1 v$ : $\star_{p}<0.01$ (ANOVA). Significantly different between sham and ARN-X: \#p<0.05 (ANOVA).

Baseline values of MAP and HR for sham ARN $-x$ and ARN- $x$ rats are shown in table 8.2 . MAP or HR did not change as a result of the remal deafferentation. The effects of a continuous ir and iv infusion of $12 \mu \mathrm{g} / \mathrm{kg} . \mathrm{hr}$ NE in $A R N-x$ and sham ARM-x rats are presented in $\mathrm{EIg}, 8.4$. Ir NE increased MAP in intact animals by $17 \pm 3 \mathrm{~mm} H \mathrm{Hg}$ (mean over 5 days of infusion) which was signiflcantly different $(\mathrm{p}<0.05)$ from the effects during iv $N E$ infusion $(10 \pm 2$ 


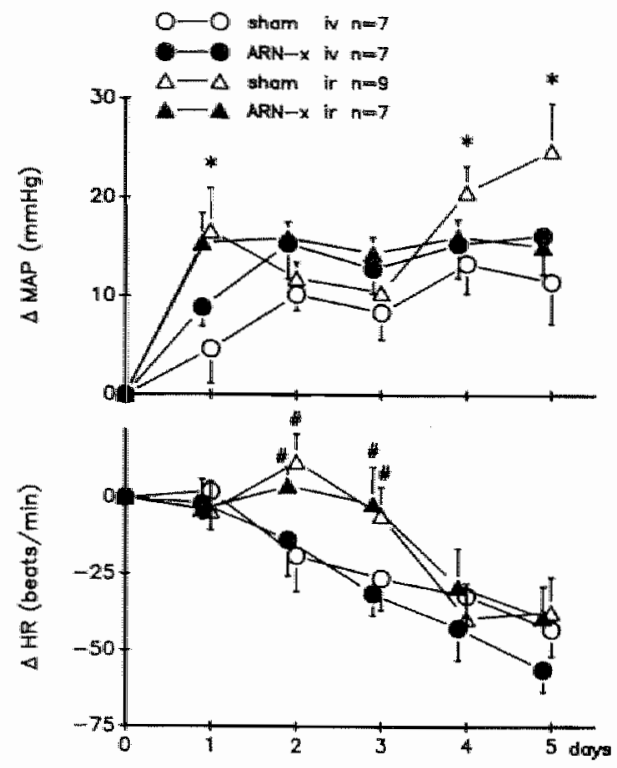

Fig. 8.4 Changes in MAP and $M R$ during ir or iv infusions of $\mathbb{N E}$ $(12 \mu g / k g$. hr) in renal deafferented (ARN-X) or sham operated rats.

Significantly different between ix and iv infused sham operated rats: * 00.05 (ANOVA).

Significantly different between ir and iv infused rats: \#p<0.05 (ANOVA).

$\mathrm{mm} \mathrm{Hg}$ ). In ARN-x rats ir infusion of NE increased MAP by $16 \pm 2 \mathrm{~mm} \mathrm{Hg}$, which did not differ from the values during ir NE infusion in intact rats. Iv NE infusion in deafferented rats increased MAP slightly more than in sham conm trols $(14 \pm 2 \mathrm{~mm} \mathrm{Hg})$.

Plasma NE concentrations (see table 8.4) on control day as well as on experimental day 4 were comparable for sham ARN-X and for ARN-x rats. After 4 days of ir ME infusion ( $12 \mathrm{wg} / \mathrm{kg} \cdot \mathrm{hr}$ ) plasma NE concentration was raised in sham ARN-X rats to levels which were not different from the levels achieved during iv NE infusion. In ARN-x rats plasma NE levels during ir NE infusions were also not different from levels reached during iv infusions.

The effects of ir NE infusion on plamsa renin levels in renal deafferented and sham operated rats are summarlzed in fig. 8.5. In renal deafferented rats baseline PRC was significantly ( 00.05$)$ reduced by $26 \%$ as compared to sham operated rats $(A R N-x(n=22): 4.5 \pm 0.5$ pmol AI/ml.hr; sham $\operatorname{ARN}-\mathrm{x}(\mathrm{n}=22): 6.1 \pm 0.6$ pmol $\mathrm{AI} / m 1 . \mathrm{hr})$. In sham $A R N-x$ as well as in $A R N-x$ rats, PRC decreased during iv infusions of $12 \mu \mathrm{g} \mathrm{NE} / \mathrm{kg} \cdot \mathrm{hr}$. Ir NE infusions of the same amount did not change PRC from control levels in sham ARM- $x$ but raised PRC by $79 \%$ in ARN-x rats. When calculated changes in PRC (day 4 day 0$)$ were compared, ANOVA revealed no differences between ARN-.x and intact rats. However, significance $(p<0.02)$ was obtained for differences beween rats infused ir and iv. 
Table 8.4. Plasma NE concentration in renal deafferented (ARN-X) and sham operated rats on control day and on day 4 of intrarenal (ir) or: intravenous (iv) $12 \mu \mathrm{g} \mathrm{NE} / \mathrm{kg} \cdot \mathrm{h}$ infusions. 3-way ANOVA (ARN-x vs. sham): NS.

\begin{tabular}{llllll}
\hline Group & $\begin{array}{l}\text { Infusion } \\
\text { route }\end{array}$ & $\mathrm{n}$ & $\begin{array}{l}\text { NE ng/m1 } \\
\text { control day }\end{array}$ & $\mathrm{n}$ & $\begin{array}{l}\text { NE ng/m1 } \\
\text { day 4 }\end{array}$ \\
\hline Sham & iv & 7 & $0.38 \pm 0.06$ & 8 & $1.65 \pm 0.12$ \\
ARN-x & iv & 6 & $0.50 \pm 0.07$ & 6 & $1.78 \pm 0.32$ \\
Sham & ir & 8 & $0.42 \pm 0.11$ & 8 & $1.73 \pm 0.20$ \\
ARN- $x$ & ir & 6 & $0.32 \pm 0.06$ & 7 & $2.83 \pm 0.56$ \\
\hline
\end{tabular}
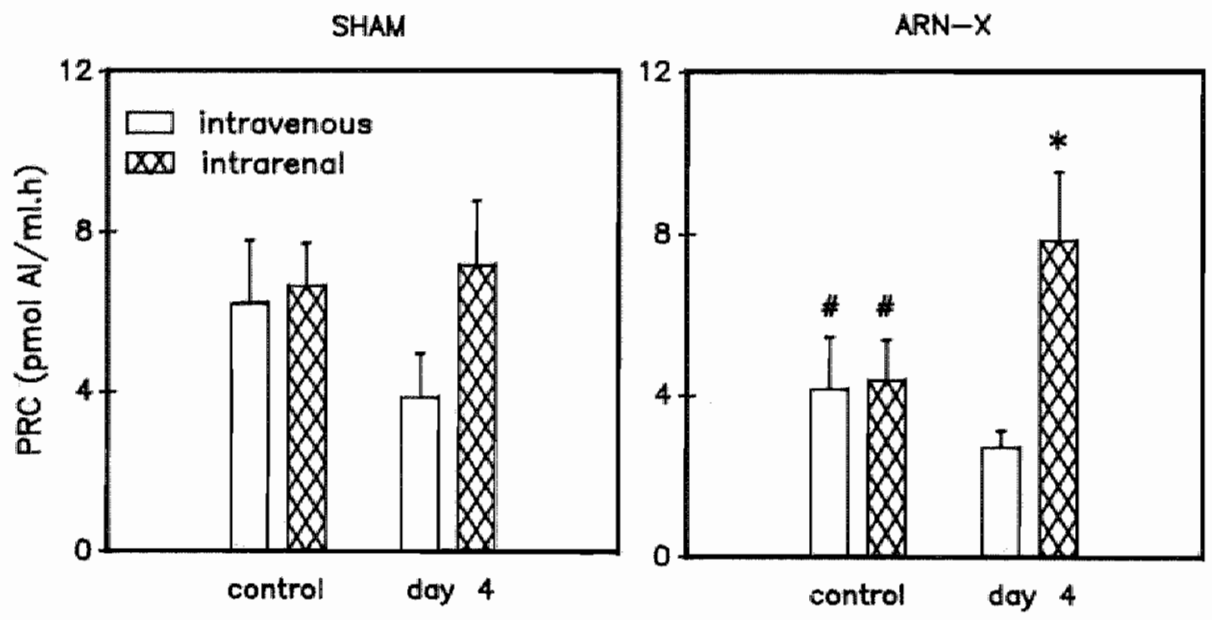

Fig. 8.5 Effects of $\mathrm{ir}$ and iv infusions of $\mathrm{NE}(12 \mu \mathrm{g} / \mathrm{kg} \cdot \mathrm{h})$ on plasma renin concentration (PRC) in renal deafferented (ARN-x) and sham opew. rated rats on control day and day 4 of infusion.

Stgnificantly different on control day between ARN-x $(n=14)$ and sham operated rats $(n=12): \# p<0.05$ (Student's t-test).

significantly different between in $(n=15)$ and iv $(n=16)$ infused rats: ${ }^{*} p<0.05$ (ANOVA).

\subsection{Discussion}

The major objective of this study was to investigate the role of renal nerves in the development of hypertension induced by continuous ir $\mathrm{NE}$ infusions. Since renal nerves are a mixed population of afferent and efferent fibers, selective methods for denervation were used. All studies were 
performed in unilaterally nephrectomized rats to exclude interference by the non-infused kidney (Kopp, 1985). RN-x resulted in an almost complete depletion of renal NE content. Approximately 3 veeks after RN-x, remal NE content was still 86\% below levels in intact rats, indicating that the denervation procedure was satisfactory. According to Kline and Mercer (1980) functional reinnervation starts within 14-21 days, but complete return of the functional efferent innervation in rats does not occur before 8 weeks after $\mathbb{R N}-\mathrm{X}$.

$A R N-X$ was verified by measuring reflex responses on MAP and HR to intrarenal $B K$ infusions. In $A R N-x$ rats ir infusion of $B K$, which is known to stimulate renal chemoreceptors (Moss, 1985; Knuepfer and Schramm, 1985), failed to increase MAP signiflicantly above levels obtained during $1 v$ infusions of the same amount. Thus, these results indicate that a transsection of the dorsal roots T9-T13 which according to anatomical data (Lappe et al., 1982; Donovan et al., 1983), destroys more than $90 \%$ of the renal sympathetic afferent projections, is sufficient for inhibiting BK induced sympathetic afferent renal reflex responses. ARN-x did not consistently prevent the effects on HR. Since dorsal roots, once cut, are unable to regenerate connections with spinal neuronal elements (Kiernan, 1979), the attenuation of this response might be caused by stimulation of the remaining sympathetic renal afferents. Alternatively, the effects on heart rate might be the result of stimulation of renal afferent nerves projecting via the vagal nerves (Gattone et al., 1986). However, up to now, there are no studies concerning the possible function of these vagal renal afferents.

RN-x reduced basal MAP significantly, confirming the results of Bencsáth et al. (1982) and Fernández-Repollet et al. (1985). However, the mechanisms through which $\mathrm{RN}-\mathrm{x}$ decreases basal MAP are not fully understood. The suggestion that the fall in blood pressure is caused by an intercuption of the ARN can be excluded by our finding that ARN-x did not decrease basal MAP, which is consistent ith observations by others (Lappe et a1., 1985; Pan et al., 1985; Wyss et al , 1986). It therefore seems that the ERN are the most important renal nerve fiber population involved in the maintenance of blood pressure in normotensive rats. It has been shown that in conscious RN-x rats, plasma aldosterone and renin concentrations were reduced (Fernăndez-Repollet et al., 1985). The latter finding could not be conflrmed in this study. RN-X, however, may also reduce blood pressure by causing diuresis and natriuresis (Rogenes and Gottschalk, 1982) or, alternatively, as was reported for spontaneously hypertensive rats (Krueger et al., 1986), by a specific dilation of the renal and mesenteric vascular beds. 
Renal deafferented rats exhibited significantly lower basal PRC levels than intact rats. Although we cannot exclude that changes in factors known to be involwed in the regulation of renin release (Zanchetti et al., 1976) may have occurred in $\mathbb{A R N}_{-X}$ rats, this finding might indicate that ARN have a tonlc stimulatory input on renin release, which is in accordance with studies in rats in which stimulation of ARN increases ERN activity (Moss, 1982; Hermansson et al., 1984; Webb and Brody, 1987). On the other hand, studies by Kopp (1985) showing that activation of ARN results in a contralateral decrease in ERN activity, do not confirm the above mentioned hypothesis. Studies on the possible influence of ARN on peripheral sympathetic nerwe activity have to be performed, preferably in conscious animals to gain more insight in these mechanisms.

RN- $x$ did not prevent or attenuate hypertension during ir NE infusions. In contrast, the increase in MAP in RN-X rats was even greater than in the control group. Since $R N-x$ is known to cause denervation supersensitivity (Kline and Mercer, 1980), exaggerated adrenergic postsynaptic renal mechanisms (D1Bona, 1982) may also explain the extra pressor response and mask the possible role of the renal nerves in this model. The fact that PRC was significantly elevated after 4 days of ir infusion of NE in RN-X rats, but not in intact rats, supports this hypothesis.

To study the role of ARN in this model, ir NE infusions were performed in renal deafferented rats and responses were compared to the effects of ir NE infusions in intact rats. As expected from the results of Kleinjans et al. (1983) ir NE infusions in intact rats increased MAP significantly more over the experimental period than iv NE infusions did. Although on experimental day 3 MAP tended to decrease, there was a steep rise in pressure on days 4 and 5 of infusion. The responses of ir $M E$ infusions in ARN-x vere at no polint in time significantly different from the effects observed in intact rats, although increases in MAP tended to diverge between the 2 groups on days 4 and 5 . Yet, the present results suggest that ARN-x does not have a significant inhibitory effect on the development of hypertension induced by ir NE infusions. We must add that the loss of the spinal afferent feedback through $T 9-T 13$ is possibly accompanied by alterations in firing rate of efferent sympathetic neurons (Sato and Schmidt, 1987), so that again clear effects could be masked due to a changed efferent sympathetic tone. An indication for this might be the reduction in basal PRC and the fact that during iv ME infusion in ARN-x rats, the increase in MAP tended to be greater than in intact rats. Effects on HR during 11 NE infusions did not differ between $A R N-x$ and intact rats. Dif- 
ferences in $\mathrm{HR}$, however, are observed between the responses to iv and in infusions. NE applied iv gradually reduced HR, but during ir infusion HR did not change for 3 days after which it decreased to comparable levels as during iv infusion. A possible explanation for these differences in $H R$ response could be that ir application of $\mathrm{NE}$ causes an activation of neural (remaining sympathetic or vagal renal afferents) or unknown humoral mechanisms, which counteract the baroreflex mediated decreases in HR.

Basal plasma NE levels in ARN- $x$ rats were not different from levels abserved in the intact rats. In contrast to what was obserwed by kleinjans et al. (1983) plasma NE levels in sham ARN-X rats did not rise significantly more during ir NE infusions than during iv infusions. The reasons for this discrepancy with the present results is unclear since exactly the same infusion protocols were used. It is common knowledge, however, that plasma NE levels do not consistently reflect sympathetic activity (Hjemdahl, 1987). In $A R N-x$ rats there is a tendency for plasma NE to be higher during it than iv NE infusion, but the difference was not statistically significant. If, indeed, ir infused $N E$ should have an excitatory influence on ARN leading to higher plasma $N E$, then plasma $N E$ levels in $A R N-x$ rats should be at least lower than in intact rats. However, it is possible that since NE is actively secreted by the kidney (Lappe et al., 1982), the differences in plasma NE levels might reflect changes in proximal tubulat function as a result of the deafferentation.

In $A R N-x$ as well as in sham ARN-x rats $P R C$ was significantly increased during ir NE infusions compared to PRC levels obtained during iv infusion. Ir application of NE may increase renin release through direct activation of beta ${ }_{1}$-receptors while during iv NE infusion renal $\mathbb{N E}$ concentrations might not reach sufficiently high levels to stimulate beta $1^{\text {-recep- }}$ tors and to overcome the inhibitory effect on renin release via stimulation of alpha ${ }_{2}$-receptors (De Leeuw et al., 1985). During ir NE infustons no significant changes in PRC due to renal deafferentation were observed, although the effect on PRC during ir NE in ARN-X rats was more pronounced than in intact rats.

Thus, in the present study, the pressor responses observed during ir NE Infusions in ARN- $x$ rats were probably dependent on the activation of the renin-anglotensin system. On the other hand, inhibition of the renin-anglotensin system in intact rats with captopril (Kleinjans, 1983) did not prevent the extra pressor response as a result of the ir NE infusions. We therefore suggest that both systems react upon stimulation with ir NE infu-... sions and that dominance of one system is assumed when the other is inti- 
palred. Similar conclusions were reached by Faber and Gettes (1987) who showed that the role of the ARN in causing a pressor reflex as a result of acute reductions in renal blood flow only could be demonstrated in rats in which the renin-anglotensin system and the sino-aortic baroreceptor reflex were inhibited. Thus, on the basis of the present results, we cannot exclude a posstble role for ARN in inducing hypertension in intact animals during it NE infusions.

The effect of selective renal deafferentation on the development of hypertension has been studied in several experimental models. ARN-x had no influence on the Induction of hypertension in Grollman renal wrapped rats (Pan et al., 1985) or spontaneously hypertensive rats (Janssen et al., 1987). In one-kidney, one-clip Goldblatt rats, sensory renal denervation prior to clipping could only attenuate the hypertension (Wyss et al., 1986). So, these studies also demonstrate that other factors besides the ARN contribute to the development of hypertension or may have compensated the effect of ARN-X.

The fact that only in one-kidney, one-clip Goldblatt rats, ARN-x results in a hypotensive response, might indicate that the involvement of ARN in the induction of hypertension is dependent on the degree to which renal blood flow is impaired. It is known that reductions in renal blood flow and perfusion pressure stimulates ARN (Moss, 1985) and that renal blood flow is relatively severely reduced in this model compared to renal blood flow in the spontaneously hypertensive rat, renal wrapped rat or in the model used in this study (Kleinjans et al., 1984). However, it is still unclear to what extent the ARN are stimulated in the several hypertensive models and to what degree $A R N$ interact with other systems in hemodynamic control.

In summary, RN-X but not ARN-x lowered basal blood pressure levels in normotensive rats, suggesting an important cole of ERN in maintaining basal blood pressure. ARN-x decreased basal plasma renin concentrations, suggesting that ARN may play a role in the control of renin release. RN-x nor ARN-* prevented or attenuated significantly the hypertension induced by ir NE infusions. These data do not confirm the hypothesis that ir applied NE increases blood pressure through activation of ARN. However, care should be taken in the interpretation of these data. Since in RN-x as well as ARN-X rats PRC was consistently increased during the ir NE infusions, a possible role for the renal nerves in this model may have been masked. Further studies are necessary to define such possibilities. 


\subsection{Introduction}

Evidence has accumulated that the development of spontaneous hypertension in rats (SHR) is characterized by an elevated sympathetic nervous system activity as measured by direct nerve recordings (Judy et a1., 1976; Coote and Sato, 1977; Thorén, 1987), responses to ganglionic blockade (Smith et a1., 1984), or by enzymatic parameters (McCarty, 1986). The cause for this sympathetic abnormality is unknown yet.

Central as well as peripheral nervous factors - especially the renal nerves (Liard, 1977; Kline et al., 1978; Winternttz et al., 1980; Krueger et al., 1986) - have been suggested to contribute to the initiation of spontaneous hypertension. Lesions of various brain structures decrease blood pressure in SHR, probably via a reduction in central sympathetic nervous activity (Ciriello et al., 1984; Van den Busse et al., 1986). Hypotensive responses have also been observed after bilateral renal denervation in these rats (Liard, 1977; Kline et al., 1978; Winternitz et al., 1980; Krueger et al., 1986). The antihypertensive mechanisms of renal denervation are still unclear. Renal denervation may lead to a hypotensive effect through alterations in renal excretory function or renin release (DiBona, 1982). In addition, renal denervation changes hypothalamic norepinephrine content (Calaresu and Ciriello, 1981; Winternitz et al., 1982) and cardiovascular responses to ganglionic blockade (Krueger et al., 1986) and electrical hypothalamic stimulation (Takeda et al., 1987) in several experimental hypertensive models. These data suggest that peripheral section of these nerwes may alter central sympathetic nervous activity.

In the above mentioned reports renal denervation techniques did not discriminate between efferent and afferent nerve flbers. This implies that the centrally mediated hypotensive effects of renal denervation can be explained by changes in renin secretion and plasma angiotensin II levels as a result of a decreased ERN activity (Ganten et a1., 1984; 0pari1, 1986). Alternatively, these effects may be mediated by the intercuption of ARN. Histological and electrophysiological studies have shown that ARM project to structures within the central nervous system that are involved in cardiovascular regulation (Ciriello and Caverson, 1987; Hebb and Brody, 1987). stimulation of ARN in conscious animals, results in a sympathetically me- 
diated pressor response (Smits and Brody, 1984; Faber and Gettes, 1987) and may lead to thypertension (Katholi et al., 1985). Furthermore, electrophyslological studies have shown that ARN in SHR are hyperresponsive to certain stimuli as compared to the effects evoked in normotensive controls (Moss, 1987), although contrasting results have been reported elsewhere (Kopp et al., 1987).

The present study was designed to determine the effects of a selective intercuption of ARN on the development of hypertension in SHR. To compare developmental and direct effects ARN-x was performed in prehypertensive and adult hypertensive rats. Additional experiments were performed to study the impact of the loss of ARN to sympathetic function and baroreflex sensitivity.

\subsection{Experimental protocols}

9.2.1 Experiment 1 : Effects of renal deafferentation im pre-hypertensive SHIRR

From each litter, young female SHR (SHR young) were divided over 2 groups. In one group of rats (23-27 days) a selective renal deafferentation of the right kidney was performed by unilateral section of the right dorsal roots of the vertebrae T8-L1. This procedure has been described in detail elsewhere in chapter 2. The second group of rats served as sham controls. These animals underwent the same surgical procedures, except for the actual rhizotomy. After $2-3$ days of recovery all rats were (left) uninephrectomized under light ether anaesthesia, to exclude a possible role of the contralateral. kidney (Kopp et al., 1987). Systolic blood pressure (SBP) was measured between $9: 00$ and $12: 00$ a.m. at weekly intervals from $3-15$ weeks, using the tall-cuff nethod. HR was derived from the arterial pulse signal with a tachograph. During this periad (week 10) rats were placed in mettabolism cages for 7 days. After 4 days of habituation daily intake of food and water was monitored for 3 days. Urine collections ( $24 \mathrm{hr}$ ) were made for determination of urinary sodium excretion. When blood pressure had reached a constant level in the sham operated SHR (week 16), all rats were instrumented with catheters (see chapter 2) to perform an experimental protocol as described below.

\subsubsection{Experiment 2 : Effects of renal deafferentation in aduIt SAR and WRY} Male 16-18 weeks old SHR (SHR adult) and WKY (WKY adult) were nephrec- 
tomized on the left side 4-5 veeks before enterling the study. To determine the effect of ARN-X, both SHR and WKY rats were divided into 2 groups with comparable blood pressures, as measured with the tail-cutit method before the surgery.

The groups of SHR and WKY vere subjected to ARN-x or sham-surgery as described above. All rats were allowed to recover for 7-10 days. After chronic instrumentation with catheters the same experimental protocol was performed in all rats.

\subsubsection{Experimental protocol}

on 3 consecutive days baseline (30-60 min) MAP and $H \mathbb{R}$ were recorded before starting the following pharmacological experiments.

\section{Verification of renal deafferentation (day 1)}

Previous studies have shown that ir infusion of BK causes an increase of MAP and HR through activation of afferent renal nerves. This reflex can be prevented by ARN-x (Janssen et al., 1987). Thus, to test the functional integrity of the ARN, rats were given $5 \mathrm{~min}$ ir and iv infusions of $B K(0.3$ and $1.0 \mu \mathrm{g} / \mathrm{min}$ of tri-acetate salt) in random order at $15 \mathrm{~min}$ intervals. Mean (2-5) min changes in MAP and HR were calculated.

\section{Heasurement of baroreflex sensitivity (day 2)}

Baroreflex sensitivity was determined as described in detail in section 2.5 .

\section{Ganglionic blockade (day 3)}

To assess the contribution of the sympathetic nervous system tone to the maintenance of blood pressure in these conscious animals, changes in MAF and HR were recorded (mean of $5-15 \mathrm{~min}$ ) after iv injection of $20 \mathrm{mg} / \mathrm{kg}$ hexamethonium (Sigma), which causes total ganglionic blockade in rats (Krueger et al., 1986).

Before any drugs were given (day 1) a $0.5 \mathrm{ml}$ arterlal blood sample was taken from all rats for determination of plasma NE levels (see section 2.9.2). At the end of the experiments, the kidney of the adult SHR was extilipated, weighed and honogenized and assessed for NE content by HPLC. Then, rats were perfused transcardially w th $10 \%$ formaline. The spinal cord was dissected and inspected under a dissection microscope. Only the animals with a complete transsection of the dorsal roots T9-T13 (about 90\% of the 
ARN; Donowan et al., 1983) were included in the data as being deafferented. In sham-ARN-x rats, all dorsal roots were intact.

Statistical analysis of the data performed as described in section 2.10 .

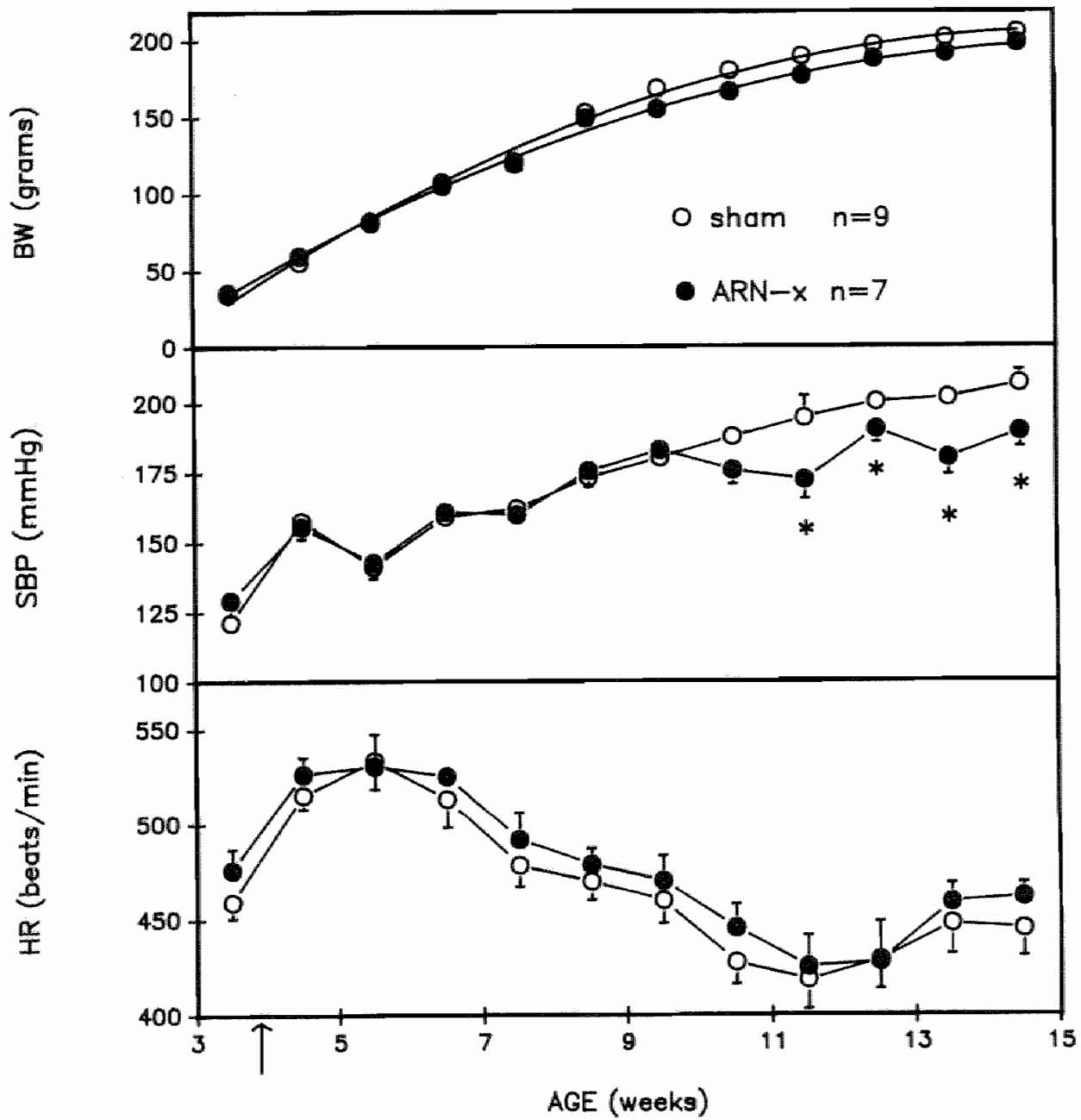

Fig. 9.1 BW, SBP and HR in renal deafferented (ARN-x) and sham operated SHR . Time of surgery is indicated by $(\downarrow)$. Significantly differenung rom sham: *p<0.05 (ANOVA). 


\subsection{Results}

A11 rats recovered remarkably well from the surgery * Wean body welght of ARN-x SHR young $(n=7)$ and the control group $(n=9)$ are presented in fig. 9.1. Up to 9 weeks the mean body weight of both groups was similar. From 10 weeks on, rhizotomized rats had a slightly but not significantly lower body welght than the control rats. Yet, during the whole experiment no obvious $\$ 11$ effects could be observed. Values for $24 \mathrm{hr}$, food and water intake and urinary wolume and sodium excretion 7 weeks after ARN-x are presented in table 9.1. No statistical differences were found in any parameter between both groups.

Table 9.1. Twenty-four hours food intake, water intake and urinary excretion of sodium and potassium in sham operated and renal deafferented (ANR-x) SHR 7 weeks after surgery. No statistical differences in anyougameter was detected (unpaired t-tests). Values are means \pm SE from 3 collection periods.

\begin{tabular}{lcclll}
\hline & $\begin{array}{c}\text { Food intake } \\
(\mathrm{g})\end{array}$ & $\begin{array}{c}\text { Water intake } \\
(\mathrm{ml})\end{array}$ & $\begin{array}{l}\text { UV } \\
(\mathrm{ml})\end{array}$ & $\begin{array}{l}\text { UNaV } \\
(\mathrm{mEq})\end{array}$ & $\begin{array}{l}\text { UKV } \\
(\mathrm{mEq})\end{array}$ \\
\hline $\begin{array}{l}\text { Sham SHR } \\
(\mathrm{n}=9)\end{array}$ & $16.9 \pm 0.6$ & $32.0 \pm 3.2$ & $17.5 \pm 0.9$ & $3.29 \pm 0.26$ & $2.48 \pm 0.28$ \\
$\begin{array}{l}\text { ANR-x SHR } \\
(\mathrm{n}=7)\end{array}$ & $15.9 \pm 1.0$ & $32.6 \pm 1.4$ & $16.0 \pm 1.2$ & $3.20 \pm 0.24$ & $2.44 \pm 0.32$ \\
\hline
\end{tabular}

\subsubsection{SBP measurements}

In $\mathbb{E}$ ig. $9.1 \mathrm{SBP}$ and $\mathrm{HR}$ of the $\mathrm{ARN}-\mathrm{x}$ SHR young and sham $\mathrm{SHR}$ young are given. SBP did not differ between the 2 groups until the rats were 10 weeks of age. In the period from 11-15 weeks the SBP ranged in control rats from 188 to $207 \mathrm{mmHg}$. The levels for the deafferented rats were silghtly (10\%) but significantly lower ( $p<0.05$ ) over this period (173-1900 mmlig). HR was at no point in time statistically different in the rhizotomized and control aninals ( $\mathrm{fig} .9 .1$ ).

\subsubsection{Direct blood pressure measurements}

Basal MAP, HR (mean values over 3 days) and plasma NE levels of al.l. rats studied in this experiment are shown in table 9.2. Although SBP in the developmental study differed in the sham control and ARN-X SAR young, MAP was identical during the direct measurements. In ARN-x SHR adult, MAP was slightly decreased. ARN-x had no effect in WKY adult . HR was not affected by 
ARll-x in either group. Plasma levels of NE vere not different between ARN-x and their sham controls in any group.

Table 9.2. Baselline MAP, HR and plasma NE concentrations in renal deafferented and sham operated SHR and normotensive WKY controls which underwent renal deafferentation (ARN-X) or sham denervation at 4 weeks (SHR young $_{\text {) or } 18 \text { weeks of age (SHR adult" WKY }}$ whIt).

Significantiyng ifferent between sham-operated and adH It sHR: *p< 0.05 (unpaired $t$-test). ND = not determined.

\begin{tabular}{|c|c|c|c|c|}
\hline & $\begin{array}{l}\text { Number } \\
\text { of rats }\end{array}$ & $\begin{array}{l}\text { MAP } \\
(\mathrm{mm} \mathrm{Hg})\end{array}$ & $\begin{array}{l}\text { HR } \\
\text { (beats/min) }\end{array}$ & $\begin{array}{l}\text { Plasma NE } \\
(\mathrm{pg} / \mathrm{m} \mathrm{I})\end{array}$ \\
\hline $\begin{array}{l}\text { SHR }_{\text {young }} \\
\text { shar wo } \\
\text { ARN-X }\end{array}$ & $\begin{array}{l}9 \\
7\end{array}$ & $\begin{array}{l}173_{ \pm} 6 \\
173_{ \pm} 8\end{array}$ & $\begin{array}{l}361 \pm 13 \\
344 \pm 9\end{array}$ & $\begin{array}{l}251 \pm 31 \\
211 \pm 34\end{array}$ \\
\hline $\begin{array}{l}\text { SHIR } \\
\text { shamplult } \\
\text { ARN - }\end{array}$ & $\begin{array}{l}24 \\
26\end{array}$ & $\begin{array}{l}182 \pm 3 \\
172 \pm 3\end{array}$ & $\begin{array}{l}330 \pm 5 \\
321 \pm 6\end{array}$ & $\begin{array}{l}373 \pm 56 \\
345 \pm 38\end{array}$ \\
\hline $\begin{array}{l}\text { WKY gdult } \\
\text { shainilut } \\
\text { ARN-x }\end{array}$ & $\begin{array}{l}8 \\
7\end{array}$ & $\begin{array}{l}125 \pm 1 \\
124 \pm 2\end{array}$ & $\begin{array}{l}346 \pm 8 \\
338 \pm 9\end{array}$ & $\begin{array}{l}\text { ND } \\
\text { ND }\end{array}$ \\
\hline
\end{tabular}

\subsubsection{Verification of renal deafferentation}

Renal deafferentation had no effect on renal NE content. In ARN-x SHR $_{\text {adult }}(n=7)$ and sham SHR adult $(n=7)$ renal concentrations were $132 \pm 22$ and $106 \pm 13 \mathrm{ng} / \mathrm{g} \mathrm{kidney}$ weight respectively. This indicates that renal efferent innerwation was not affected by ARN-x. BK was infused ir to evoke ARN dependent increases in MAP and HR. In all sham operated rats Bk infused ir at a rate of 0.3 and $1.0 \mu \mathrm{g} / \mathrm{min}$ increased MAP significantly (p<0.001) compared to levels reached during iv infusions of the same amount (see fig. 9.2). In ARN-X rats, the same dose of BK falled to increase MAP significantly more during ir than during iv infusions. Interestingly, comparison of the pressor responses in sham operated rats revealed that ir as well iv $B K$ infusions led to significantly ( $p<0.001$ ) more potent pressor responses in intact SHR than in intact WKY.

\subsubsection{Deternination of baroreflex sensitivity of $\operatorname{HR}$}

Sympathetic and vagal barocetlex control of HR were assessed respectively by injections with sodium nitroprusside and phenylephrine. The results are sumarlzed in figure 9.3. Baroreflex sensitivity was higher in 


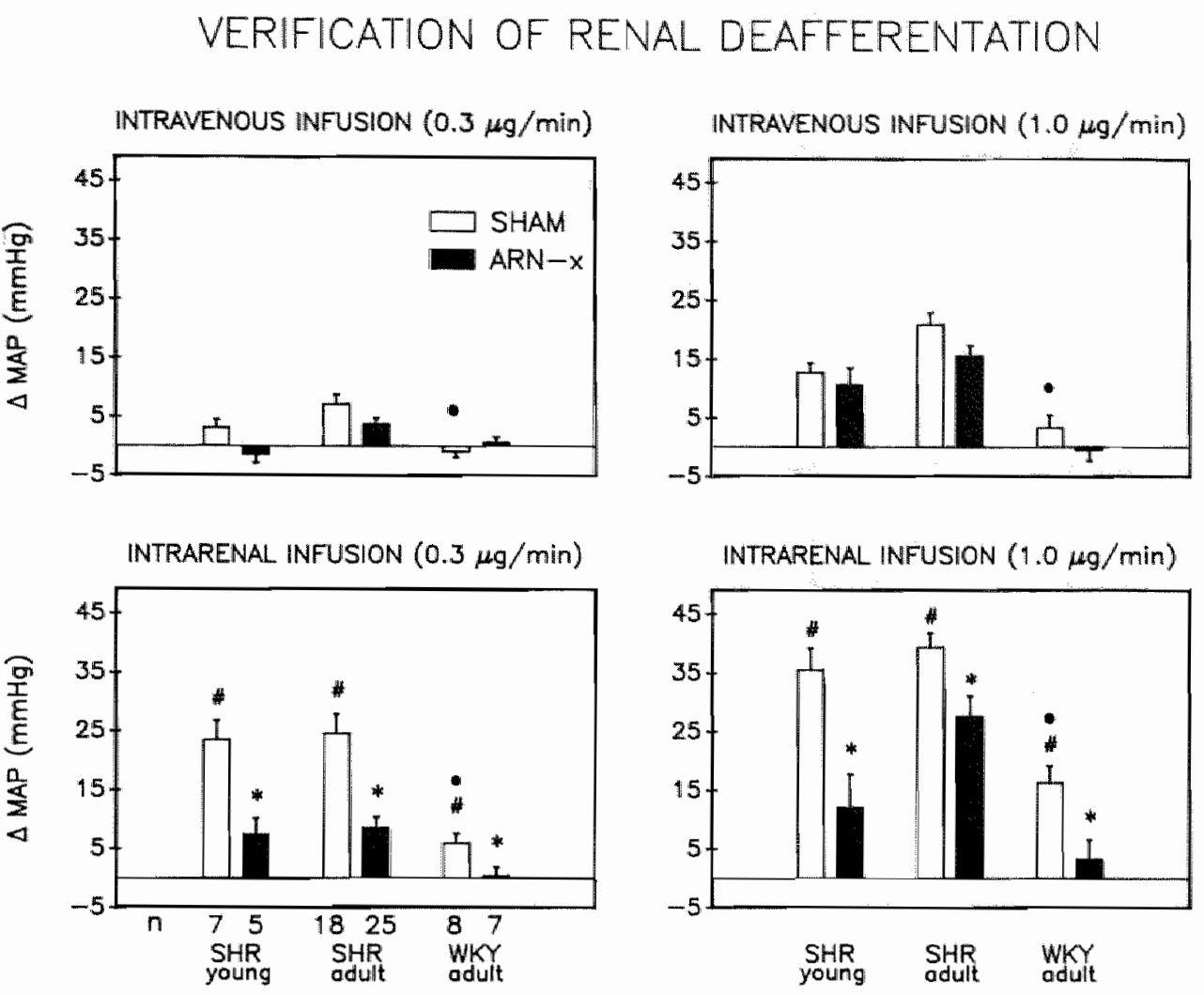

Fig. 9.2 Changes in MAP and HR during ir and IV BK infusions in renal deafferented $(A R N-x)$ and sham operated rats at 4 (young) and 22 (adult) weeks of age.

Significantly different between sham and ARN-x: $* 0<0.01$.

Signiflicantly different between iw and ir infusion: \#p<0.01.

Significantiy different between SHR and WKY: $\bullet$ p<0.01.

ARN-X SHR young than in sham controls. The difference was statistically significant $(\mathrm{p}<0.05)$ both for phenylephrine $(0.68 \pm 0.09 \mathrm{vs} .0 .40 \pm 0.07 \mathrm{~ms} / \mathrm{m}$ migh) and sodihim nitroprusside $(1.19 \pm 0.11 \mathrm{vs} .0 .66 \pm 0.07 \mathrm{~ms} / \mathrm{mmHg})$ induced baroreflex responses in heart rate. In SHR adult renal deafferentation caused a significant $(\mathrm{p}<0.05)$ increase in the phenylephrine induced baroreflex effect (ARN-X $0.52 \pm 13$ us. sham $0.27 \pm 0.03 \mathrm{~ms} / \mathrm{mmHg}$ ). ARN-x did not affect baroreflex sensitivity in WKY adult. 

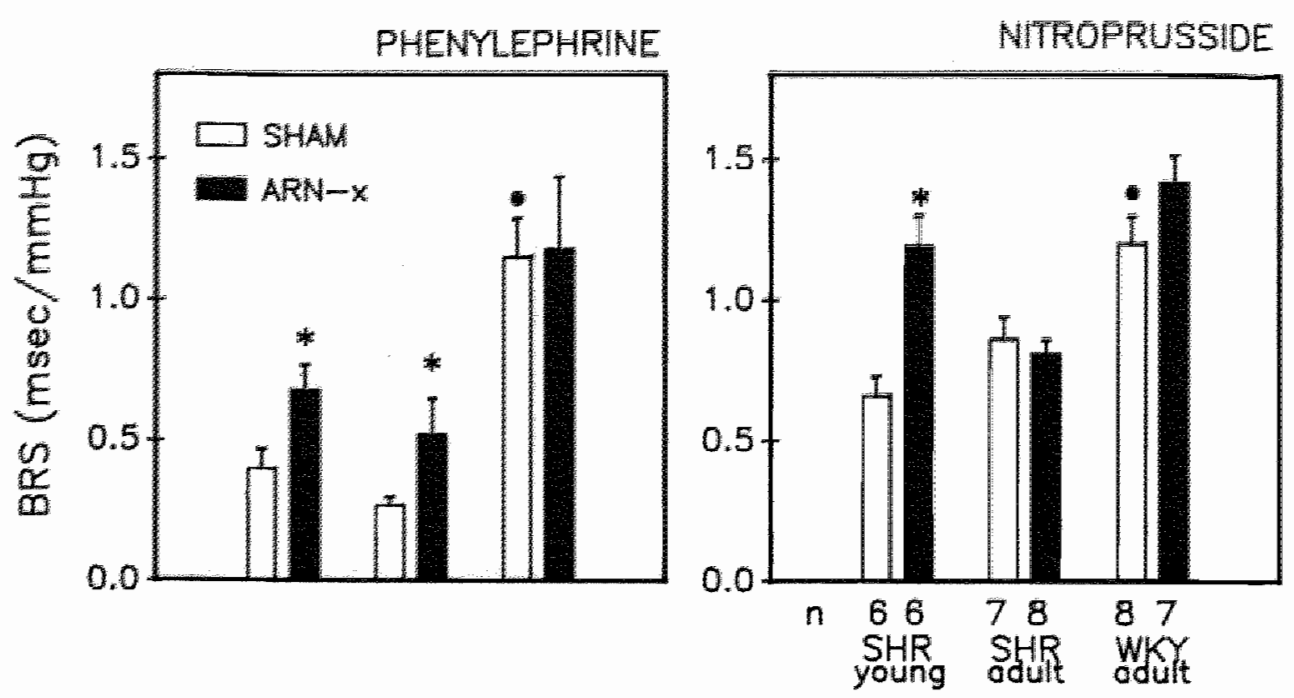

Pig. 9.3 Baroreflew sensinivity to phenylephrine and mitroprusside injections in renal deafferented (ARN-X) and sham operated rats at 4 (young) and 22 (adult) weeks of age.

Signifleantiy different between sham and ARN-x: ${ }^{*} \mathrm{p}<0.05$.

Signiflcantly different between WKY and SHR: $\bullet<<0.01$.

RESPONSE TO GANGLIONIC BLOCKADE

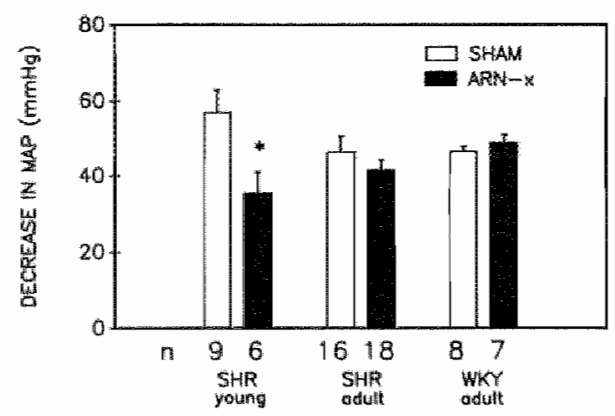

Fig. 9.4

Effects of ganglionic blockade (hexamethonitum) on MAP in renal deafferented (ARN-X) and sham operated rats at 4 (young) and 22 (adult) weeks of age. Significantly different from shan: $\star p<0.05$.

\subsubsection{Responses to ganglionic blockade}

MAP responses to ganglionic blockade are shown in fig. 9.4. Intravenous bolus infection of hexamethonium decreased MAP significantly ( $<<0.05$ ) more in sham operated $\mathrm{SHR}_{\text {young }}(57 \pm 5 \mathrm{~mm} \mathrm{Hg})$ than in $A R N-x$ SHR young $(35 \pm 6 \mathrm{~mm}$ $\mathrm{Hg}$ ). ARN $-\mathrm{x}$ had no effect on changes in MAP induced by hexamethonium in SHR adult and WKY adult rats. 


\subsection{Discussion}

The purpose of this study was two-fold. First we examined whether ARN contribute to the development and maintenance of hypertension in SHR. Secondly, it was determined whether ARN-x affected the development of an exaggerated sympathetic nervous system activity and reduced baroreflex sensitivity, which are known to develop in parallel with hypertension in SHR. Complete renal denervation techniques by surgical stripping of the renal blood vessels cannot discriminate between the afferent and efferent nerve population. We therefore performed a selective transsection of the ARN by cutting at least the dorsal roots T9-T13, which, according to anatomical data (Donovan et al. 1983), contain approximately $90 \%$ of the right renal afferent projections. This procedure results in a functional deafferentation as verified by the attenuation of afferent renal nerve dependent reflex effects evoked by intrarenal bradykinin infusions in conscious rats. ARN-X does not affect the efferent renal innervation, since renal NE content was not different in sham and deafferented SHR.

This study demonstrates that ARN- $x$ does not prevent the development of hypertension. In rhizotomized ARM-x SHR young and sham-operated rats, the time course of blood pressure increase was similar until the animals were 10 weeks old. From the 12 th week on, SBP as measured with the tailcuff method was slightly but significantly lower in the deafferented rats, although direct arterial blood pressure measurements indicated that the resting MAP and HR were not different in both groups.

The course of the development of hypertension after ARN-x is quite different from the pattern observed after complete renal denervation. The latter method results in a delay of onset of hypertension by approximately 3 weeks (Liard, 1977). The fact that development of hypertension is only delayed and not prevented has been explained on the basis of reinnervation, which has been shown to start within days following denervation and to parallel development of hypertension (Kline et al., 1980; Winternitz et a1., 1980; Cuche and Liard, 1981).

ARN-X performed in SHR adult with established hypertenstion had no important influence on resting blood pressure and HR. MAP was sllghtly ( $5 \%$ ) but significantly reduced in ARN-X SHR adult as compared to sham SHR aduIt" This hypotenstve effect of ARN-X is small in comparison with the $20-27 \%$ reductions in arterial blood pressure after complete renal denervation in adult SHR (Rrueger et al., 1986). In other reports, howewer, renal denervation failed to induce a hypotensive effect in sin when hyperterision was 
established (Minternitz et al., 1980). Nevertheless, our data suggest that the reported hypotensive effects of complete renal denervation in young as wel1. as in adult SHR (Liard, 1977; Kline et al., 1978; Winternitz et al., 1980; Winternitz et al., 1982; Lee and Walsh, 1983; Krueger et al., 1986; Takeda et al., 1987) are mainly due to the loss of the ERN.

The contribution of the sympathetic nervous system to arterial blood pressure as determined by ganglionic blockade with hexamethonium did not change in SHR and WKY rats when ARN-X was performed in adult animals. However, renal deafferentation in prehypertensive SHR young reduced the blood pressure responses to ganglionic blockade significantly as compared to walues reached in sham operated SHR young. Thus, ARN may contribute to the development of sympathetic nerwous system activity, but are not invalved in the malintenance of this system, when hypertension is established in these rats. The latter part of this conclusion is in accordance with a study from Kline et al. (1982) who reported that complete renal denervation in adult SHR did not alter central or peripheral noradrenergic activity.

Krueger et al. (1986) showed that in adult SHR following complete renal denervation, blood pressure was significantly less dependent on central sympathetic tone. Comparison of our results with these obserwations suggests that this effect of complete renal denervation is once more mediated through the loss of the ERN. Thus in adult SHR, ERN in contrast to ARN, have a tonic contribution to the maintenance of blood pressure and sympathetic tone. This deduction is supported by evidence from electrophysiological studies showing that ERN activity in SHR is increased (Judy et al., 1976; Coote and Sato, 1977; Lundin et al., 1984; Thorén, 1987) and renal blood flow is decreased (Evenwel et al., 1983) as compared to normotensive control animals. ARN may contribute to the development of the increasing sympathetic tone which, according to Judy et al. (1976), runs in parallel with the course of blood pressure in SHR.

The mechanisins through which a loss of the ARN population causes the afore mentoned effects are not fully understood. The results from the present studies provide evidence for the fact that in SHR, besides central (Clrlello et al., 1984; Van den Buuse t al., 1986) and spinal (Schramm and Chornoby, 1982) structures, also peripheral sensory mechanisms may contribute to central sympathetic tone. The hyperreactivity of SHR to sensory (stressfull) stimuli (Chiuch and koplin, 1978; Kvetnansky et al., 1979) and the fact that sensory deprivation (Lais et al, 1974; Hallback, 1975) and treatment with capsaicine (Virus et al., 1981) have hypotensive effects in SHR, can be interpreted as evidence for the involvement of hyperexcitable 
peripheral mechanisms. Furthermore the exaggerated sympathetic responses of SHR in comparison to WKY, when BK is infused ir and $1 v$, or injected into the carotid artery (Buñag and Takahashi, 1981), can also be explained by an increased sensitivity of sensory mechanisms in these rats. In electrophysiological studies ARN of SHR are known to be hyperreactive as compared to normotensive controls when an uschemic stimulus is applied to the kidney (Moss, 1987). This characteristic response to hypoxia is likely not rem stricted to the renal afferents only. Trezbki and co-workers (1982) reported that arterial chemoreceptor drive is augmented in SHR and also in hypertensive humans, leading to increased ventilatory and hemodynamic pressor responses when acute hypoxia was induced. Taking these data together, we suggest that hypersensitivity of several sensory mechanisms may contribute to the early changes in sympathetic and cardiovascular variables in SHR.

Sympathetic and vagal baroreflex control of HR but not of renal sympathetic nerve activity (Thorén, 1987) is reduced in SHR (Coote and Sato, 1977; Struyker-Boudier et al., 1982). The reduced sensitivity of baroreflex control of $\mathrm{HR}$ is due to an impaired development of a normal baroreflex sensitivity (Struyker-Boudier et al., 1982) and most likely associated with the increase in central sympathetic tone (Coote and sato, 1977) and structural changes in aorta riorphology (Krieger, 1986). The present results indicate that the vagal reflex arch to the heart is sensitized following ARN-x in SHR young and SHR adult" We propose that this sensitization of baroreflex control of HR is due to the loss of a tonic intibition of ARN input to central structures which convey baroreflex information and to which ARN are known to project (Felder, 1986; Ciriello and Caverson, 1987; Webb and Brody, 1987). In ARN-X SHR young but not in the ARN-x SHR adult the sympathetic reflex arch has also increased in sensitivity even to values comparable to those in WKY rats. Since in these animals sympathetic tone is set to a lower level, it is likely that an adequate response of HR occurs to counteract the induced hypotension. In adult SHR in which the sympathetic nervous system activity has not decreased following ARN-x, HR responses to hypotension are impaired since sympathetic influence is already at a higher level.

Summarizing, ARN are not essential in the development and malintenance of hypertension in SHR but do affect the development of central sympathetic function and baroreflex sensitivity of heart rate in SHR. "This suggests the existence of an imbalance of sympatho-excitatory sensory mechanisms, and sympatho-inhibitory baroreflex mechanisms in SHR. The causal link between these mechanisms remains to be determined. 

In this thesis, experimental data have been presented on the significance of afferent renal nerves in circulatory control under a variety of experimental conditions. The purpose of this chapter is not to repeat the discussing of these findings in detail, but to present a concept of what the physiological and possible pathophysiological functions of these afferent nerves in body fluid homeostasis are.

To understand the functional significance of the afferent renal nerves in circulatory control, the following points should be noted. Afferent renal nerves are a part of the autonomic nervous system, which has been called "the integrator of body function in its totality" (Brooks, 1983, 1987). The autonomic nerves are involved at several levels. They may influence local cellular activity and coordinate the cellular pracesses within an organ or organ system. Moreover they are under the direction of control centers which organize the activities of organ systems through the autonomic nerves. Neuro-anatomical and neurophysiological studies have shown that afferent renal nerves project to various levels of the autonomic nerwous system. Afferent renal nerwes, with some overlap, are segmentally distributed throughout the spinal cord, where they modulate preganglionic efferent nerve activity. They might even, as suggested by Dector and Weems (1981) directly influence postganglionic efferent nerve activity. At supraspinal levels ARN terminate in nuclei of the central nervous system which are known to be involved in cardiovascular regulation (Webb and Brody, 1987; Ciriello and Caverson, 1987).

Since each segmental level of the autonomic nervous system exhibits its own rules (Calaresu, 1982), the final reflex pattern induced by changes in ARN activity is most likely the result of integration of this information with the ongoing activity at spinal and supraspinal levels (Felder, 1986). According to Ross et al. $(1984)$, under normal conditions sympathetlc preganglionic nerve activity depends to a large degree on descending excitatory and inhibitory supraspinal information. However, sptnal systems, left to themselves, are capable of generating substantial activity and can alter their activity upon spinal afferent input (Schramm and Livingstone, 1987). Thus, the influence of ARN on sympathetic outflow is dependent on the interaction with central descending activity and nervous activity arising from other visceral and somatic structures (Jânig, 1988). Specifi- 
city in the efferent output depends on the organization of the reflexes and the quality and quantity of afferent input.

In most of the studies on ARN, individual parts and processes of the ARN reflex pathways have been examined under conditions and using methods which were relatively far from those occurring under normal physiological conditions (anesthesia, electrical stimulation). To understand the role of the afferent renal nerves in the control of body fluid homeostasis, their possible interactions should be considered in the whole animal. For this reason, most of the experiments in this thesis were performed in conscious unrestralned anümials.

To determine the role of ARN in body fluid homeostasis in consclous unrestralned animals, three different approaches were used. Hemodynamic reflex effects vere examined during acute as well as prolonged stimulation of the ARN. Altematively, hemodynamics were studied in rats which were subjected to a selective surgical interruption of ARN. The results of those studies were compared with the effects occurring in intact animals. It should be recognized that although the latter method inhibits hemodynamic reflex effects induced by ir infusions of BK as shown in several chapters, a complete renal deafferentation, due to the complex arrangement of the renal innervation is almost impossible to achieve (Drukker et all., 1987). Consequently, most effects were not fully abolished but rather significantly attenuated in renal deafferented rats. The dorsal rhizotomy does furthermore eliminate afferent information arising from somatic structures and other visceral structures. The influence of the loss of this non-renal afferent input on the present results is difficult to judge and may depend on the experimental protocol. Nevertheless, the clear adwantage of this method is that in contrast to complete renal denervation techniques, dorsal rhizotomy results in a more permanent loss of renal sensory information and does not affect the efferent renal nerves. Taken together, we are confident that our approaches allow formulation of conclusions on the functional role of the sensory renal nerves.

In deduction from the results presented in this thesis in combinaw tion with data obtained from 11terature, we propose that ARM are involved in clrculatory control by modulating efferent sympathetic merve activity at the level of the kidneys (renorenal reflexes) as well as at the level of other organ systems (renosystemic reflexes).

To out opintion, the most specialized function of the ARN is their modulatory influence on renal excretory function. We propose that because of their unique anatomical location and their sensory properties ARN con- 
tinuously monitor renal electrolyte transport at several levels of the tubular transport mechanisms. They may contribute to the maintenance of the renal osmotic gradient and change their activity when changes in the osmotic composition of the renal filtrate occur. As suggested in chapter 6, they may have a special sensitivity for sodium and induce compensatory changes in ERN activity when sodium output is altered. These reflexes can occur at spinal and supraspinal levels. They induce compensatory changes in the contralateral kidney as well as in the ipsilateral kidney, although in most studies the latter effects are often masked by the stimulus Itself. According to the electrophysiological data of Rogenes (1982) and the observations of the present experiments and those of others (see Kopp, 1985), renorenal reflexes may be inhibitory as well as excitatory. Despite their unique sensitivity, the regulatory capacity of renorenal reflexes is probably 10w. The special task of ARN may be their fine-tuning role in the control of ERN activity under conditions which are within a normal physiological range. As suggested in chapter 6 , and indicated in studies with completely denervated kidneys, larger compensatory changes in renal excretory function are under control of other more powerful mechanisms. The ARN induced adjustments in ERN activity may take place in the absence of renal and systemic hemodynamic changes. Several studies (DiBona, 1982; Beers et al., 1986; Osborn and Kinstetter, 1987) have provided evidence that small changes in ERN activity may alter renin secretion and tubular reabsorption without changes in GFR and ERPF. The observation in the present thesis that in normotensive renal deafferented Wistar rats plasma renin levels were significantly lower than in intact rats might indeed be a reflection of the control of ARN on basal efferent renal neurogentic tone. More subtle experiments are needed to give a specific description of the influence of ARN on renal function. In this regard, it is important to determine the exact stimuli and physiological factors that modulate ARN activity under normal physiological conditions. Studies using antibodies to the sensory endings of the renal nerves to demonstrate their exact anatomical location may help elucidating this problem.

As has recently been suggested by Caverson and Ciriello (1987, 1988), ARN may have a specialized central function in stimulating vasopressin release. ARN sense intrarenal osmotic disturbances independent of changes in plasma osmolarity. Thus, ARN may modulate AVP release before hypothalamic osmosensors become activated. The existence of such a reflex in conscious unrestrained animals has not yet been demonstrated, but teleologically its existence makes sense. Future research should define such a 
posslbie role.

Whether the above mentioned spectalized functions of the ARN are of importance in maintalining normal electrolyte and fluid balance in humans is not known. Nevertheless, observations made in patients exhibiting various degrees of autonomic fallure, can be interpreted as being caused by the loss of these autonomic reflexes. Several examples of these disturbances have been discussed in detail by Wilcox (1983) and Gottschalk et al. (1985). It is obvious that the above discussed renorenal reflexes can play an limportant role in the long-terin control of arterlal pressure. According to Kopp et al. (1987, 1988), these mechanisms contribute to the development of hypertensilon in Goldblatt $2 \mathrm{~K}-1 \mathrm{C}$ and spontaneously hypertensive rats.

Wth regard to the renosystemic reflexes, the present thesis has dealt primarlly ith thelr involvement in short-term cardiovascular control. Barber and Moss (1985) showed that renal chemosensitive nerves raise their activity when renal blood flow is gradually reduced. Under conditions of ischemia, they increase their activity up to 500\% (Recordati, 1978; Moss, 1987). In conscious rats, acute reductions in renal blood flow increase blood pressure sympathetically through ARN (Faber and Gettes, 1987). On the basis of these experiments and data presented in this thesis (chapter 3 and 4) we suggest that these nerves play a regulatory role in cardiovascular control under conditions of hypoxia. These properties are probablyr not unique for ARN but thay be a general feature of most visceral, thoracic and somatic afferents (see chapter 4). There are many reports that activam tion of sympathetlc afferents gives profound cardiovascular reactions (MalLlani, 1982; Osterziel et al., 1984; Hagenan et al., 1987; Longhurst et a1., 1987; ordway et al., 1988).

It is tempting to speculate that these reflexes subserve mechanisms which control organ perfusion. Autoregulatory mechanisms counteract a fall in organ blood tlow by releasing vasodilatory substances such as bradykinin (BK) and adenostme. At the same time, however, as is indicated in the present experiments, the same substances may act as chemoreceptor stimulants to Increase blood pressure. It is unclear how these mechanisms interreact and under wich circumstances one of them prevails. Infusion of minimal anounts of BK $(0.1$ nmol/min) in organs with a relatively high blood flow can evoke pressor responses. Thus, concentrations in the vicinity of the nerve endings needed to raise afferent nerve activity are probably much lower. This suggests that under normal physiological conditions local endom genous BK levels may be responsible for ongoing afferent nerve activity, thereby contributing to the setting of efferent sympathetic tone to main- 
tain perfustion pressure. This latter theory, however, stands or falls with the concentrations of BK generated under normal physiological conditions in vivo. Since $B K$ is rapidly unactivated by several peptidases, its exact local concentration is diffucult to judge (Regoli and Barabe, 1980).

Most of the observations discussed above have been obtained from acute studies. It now becomes important to determine whether these mechamisms contribute to long-term regulation of blood pressure. Experimental evidence for such a role is scanty (see chapter 5).

The question whether afferent renal nerve dependent sympathetic reflexes or comparable reflexes arising from other structures play a role In the development and maintenance of several forms of hypertension is st111 under debate. Many investigators (for overview, see Genest et al., 1983) agree that an elevation of sympathetic activity is involved in this process but disagree about the mechanisms how this hyperactivity develops and induces high blaod pressure.

We bypothetize that the sympathetic reflexes described in this thesis may induce hypertension when they are activated chronically.

To elevate blood pressure permanently, baroreflexes and other reflexes which may counteract the sensory nerve induced pressor response are necessary to be reset. One of the characteristics of the sensory nerve dependent reflexes is that an increase in blood pressure is accompanied by an increase in heart rate. Consequently, this means that these reflexes can overrule baroreceptor function. In many experimentally induced forms of hypertension, baroreflex resetting is secondary to the effect of the rise in blood pressure and may be the result of structural changes in the aortic vessel wall (Krieger, 1986). However, under some circumstances, the resetting is so fast that it is likely that central nervous mechanism are involved (Coleridge et al., 1981; Kunze, 1981). Similar processes as the latter may occur during activation of the afferent nerves studied in this thesis. On the basis of the experiments performed on SHR (chapter 9) we have suggested that an increased input from sympathetic afferent nerves can suppress baroreceptor function. Elimination of part of afferent sympathetic input to the nuclei relaying baroreceptor afferents can facilitate baroreceptor function. This implies that although sensory nerve dependent sympathetic reflexes can be generated at spinal levels (Faber, 1987), a central component of these reflexes is a prerequisite to induce permanent changes in blood pressure by interference with central baroreflex mechanisms. Structures in the cortex (Verberne et al., 1988), hypothalamus (Brody et al., 1980; Webb and Brody, 1987) as well as medulla (Palkovits 
and Zaborsky, 1977) are probably involved.

Another feature of the sensory nerve dependent reflexes wich to our opinion is of major importance in the development of hypertension is the fact that activation of the sympathetic afferent nerve reflexes is characterized by an increased efferent renall sympathetic nervous activity, resulting in increased tubular sodium reabsorption (DiBona, 1982). Without an Intact efferent renal Innervation, the sympathetic increases in blood pressure may be blunted by the accurrence of pressure diuresis. As described in the introduction, many forms of experimental hypertension (see table 1.2) fall to develop when the kidney has been completely denervated. The development of thypertension is in many of those cases delayed onlly. Blood pressure rises in parallel with the reinnervation of the kidney (Kline et a1., 1980).

Summarizing, activation of sympathetic afferents increases blood pressure, inhibits the counterregulatory influence of the baroreflex and prevents the occurrence of pressure diuresis by enhancing tubular reabsorption. According to theory (Folkow, 1982) these mechanisms should lead to hypertension by inducing structural changes in the vasculature when they are chronically activated. Such crucial experiments still have to be performed. As suggested in chapter 5, an intermittent ir BK infusion could be a method to test this hypothesis.

There is scanty evidence for the involvement of afferent reflexes in the initiation of different forms of experimental hypertension. The most positive evidence for the involvement of ARN comes from studies on renovascular hypertension in $1 \mathrm{~K}-1 \mathrm{C}$ rats. Wyss et al. (1986) showed that renal deafferentation one week prior to renal artery clipping significantly attenuated (for minimally 8 weeks) the rise in blood pressure which was observed in clipped sham operated rats. The fact that the blood pressure level in ARN-X elipped rats was elevated above levels in control non-clipped rats suggest that also other factors contribute to the development of high blood pressure. When rats ith established 1K-1C hypertension were subjected to ARN-x, systolle blood pressure fell only for 2 weeks but returned to prethizotomy levels in the 3rd week (Opar11,1986). These data indicate that ARN have an important contribution in the development and maintenance of IK-1C hypertension. In Grollman hypertensive rats, renal deafferentation did not affect the progressive rise in blood pressure induced by renal wrapping (Pan et al. 1985). Also wen hypertension was established, ARN-x did not influence blood pressure levels. Thus, afferent renall nervous mechantsins do not seem to contribute to hypertension in this model. 
The involvement of the ARN in the development of hypertension induced by continuous ir $N$ infusions is less clear. The results presented in chapter 8 suggest that the extra pressor responses observed during ir $\mathrm{NE}$ infusions is partly dependent on activation of the ARN but also partially dependent upon activation of the renin-angiotensin system.

The significance of the ARN in the development and maintenance of spontaneous hypertension in rats has been extensively described in chapter 9. Deafferentation studies showed that ARN are not essential in the development and maintenance of spontaneous hypertension but do affect the development of central sympathetic function and baroreflex sensitivity. These data suggest the existence of an imbalance of sympatho-excitatory sensory mechanisms and sympatho-inhibitory baroreflex mechanisms. The causal link between these mechanisms remains to be determined. It may be hypothetized that the increased sensitiwity of chemoreceptors in SHR (chapter 5,9; Moss, 1987; Fukuda et al., 1987) contributes to the development of sympathetic nervous system hyperactivity and increased vascular resistance. Whether this reflects a genetic defect in the sensory receptors or in the processing of information is unknown.

The above data on the involvement of ARN in the several models of experimental hypertension lead us to the following conclusions. ARN contri... bute to a considerable amount to the setting of the blood pressure level only in models in which renal blood flow is severely impaired. One may speculate through a reduction of renal blood flow ARN become activated to ellcit an increase in efferent sympathetic activity to elevate renal perfusion pressure directly as well as by reflexly stimulating renin release. When a clip or other obstruction (atherosclerotic plaque) prevents the return of renal blood flow to normal, the increased angiotensin production and sympathetic increases in renal vascular resistance prolong the hypoxic citcumstances and increase ARN activity and systemic blood pressure even further. This feed forward loop will continue increasing blood pressure until normoxic conditions in the kidney are reached or until other systems counteract it. Whether these mechanisms indeed take place in human renovascular hypertension is unknown. Yet, in several clinical studies, neurogenic components in this disease have been observed (Oparil, 1986; Mathias et al., 1987).

According to this theory and to the observations in chapter 4 that renal and visceral afferent nerves have similar properties. fit would be reasonable to assume that also clipping of other arteries (e.g. superior mesenteric artery or femoral artery) would ultimately induce hypertension. 
We suggest that this manoeuver indeed stimulates afferent activity to increase perfusion pressure. Yet, the activity of these systems declines in time since the visceral and femoral vascular beds can restore blood flow by shunting of the blood from ather arteries supplying the organ system. For this reason Goldblat et al. (1934) probably did not find hypertension after clipping the superior mesenterlc artery in dogs. Also when organ blood flow can be restored in any other way (e.g. formation of collaterals or hormonal actions) the activation of the afferent nerve system is not necessary anymore. Thus, systemic blood pressure 111 not rise and deafferentation studies will give negative results.

In conclusion, this thesis examines the physiological and pathophyslological aspects of the involvement of renal sensory mechanisms in circulatory control. The studies indicate that these renal sensory mechanisms contribute to the control of normal renal function. Furthermore, evidence is presented suggesting that renal as well as sensory nerve activity arising from other peripheral structures may contribute to the maintenance of organ blood flow by increasing perfusion pressure. Under certain conditions, these sensory nerves may be involved in the initiation of hypertension. The present studies emphasize the physiological and pathophysiological importance of afferent nerwous mechanisms and may open new perspectives in our understanding and therapy of diseases of the cardiovascular system. 
The kidney is thought to play a key role in the regulation of body fluid homeostasis and blood pressure by controlling the excretion of water and electrolytes. The contribution of neural mechanisms to these processes has been recognized only recently: Studies in this field are malnly concerned with the functional significance of the efferent nerves wich conduct information to the kidney. Little attention has been pald to the significance of nerve impulses arising from the kidney. The concept that the kidney is a sensor organ involved in circulatory control is currently gaining more support. The research described in this thesis has been performed to test the hypothesis that the kidney wilits sensory or afferent nerves (ARN) influences effector mechanisms that play a key role in the regulation of body fluid homeostasis and blood pressure. A detalled introduction to this hypothesis can be found in chapter 1 .

The functional significance of the ARN has been studied in rats via different approaches. The materials, techniques and methods that were applied are described in chapter 2. ARN impulse activity was recorded electrophysiologically during intrarenal infusions of bradykinin, adenosine and norepinephrine. It was shown (chapter 3) that ARN increase their firing rates when these agents are infused into the renal blood stream. The same local infusion thethod was applied to study the acute hemodynamic effects of activation of ARN in conscious unrestrained rats. In order to obtain information about the specificity of the ARN induced reflexes, the results were compared to those elicited by lacal infusion of the same agents into a side-branch of the superior mesenteric artery. It was found that for all agents applied, renal as well as mesenteric afferents (chapter 4), in general, evoke similar sympathetically mediated pressor responses. This: indicates that the renal and mesenteric afferents share not only common reflex pathways but also a similar physiological function. It is suggested that these sensory nerves play a role in the maintenance of organ perfusion. Furthermore, it was hypothetized that a continuous activation of these reflexes could contribute to the mechanisms leading to hypertension. This latter hypothesis was tested by examining the hemodynamic effects of prolonged periods of intrarenal bradykinin infustons (chapter 5). Although blood pressure can be effectively elevated by this method, probably much longer infusion periods are needed to induce permanent changes in blood pressure. 
In chapter 6, the influerce of ARN on renal sodium excretion was examined during the transition from a period of normal to a period of low sodium intake in control rats as well as in rats which vere subjected to surgical elimination of the ARN (ARN-X). The study revealed that ARN are not necessary in the maintenance of sodium balance at both intake levels, but that they are able to correct a short-lasting transient increase in sodium excretion. Chapter 7 describes the possible contributions of ARN dependent reflex mechanisms in the cardiovascular adaptations wen urine flow is increased by diuretics. Denervation studies showed that ARM are not involved in the mechanisms that induce acute increases in renal and later increases in systemic vasicular resistance when furosemide or acetazolamide was applied. The mechanisms of the acute increase in renal wascular resistance upon administration of these drugs is still unknown. The later generallzed increase in vascular resistance was shown to be renin dependent.

The contribution of ARN to the development of hypertension in rats made hypertensive by chronic intrarenal norepinephrine infusions (chapter 8) and spontaneously hypertensive rats (chapter 9) was examined by ARN $-x$ prior to the initiation of hypertension. ARN-x did not prevent nor attenuate the increase in blood pressure in the first model significantly. The results suggest that a concomitant activation of the renin-angiotensin system may have masked a possible role for ARN in the intrarenally norepinephrine-induced hypertensive rats. In SHR, afferent in contrast to efferent renal nerves are not essential to develop or maintain a high blood pressure. However, ARN-x affects the development of sympathetic function and baroreflex sensitivity. This suggests that these rats exhibit an imbalance between sensory sympatho-excitatory and baroreflex sympatho-inhibitory mechanisms. The causal link between these mechanisms in relation to spontaneous hypertension remains to be determined.

Finally, chapter 10 tries to combine all observations and to place them in one context, explaining how the kidney as a sensor organ is interrelated wh effector mechanisms that control the circulation in physiological and pathophysiological states. 
In de klassieke medische literatuur speelt de nier een sleutelral in thet konstant houden van de lichaamsuloeistoffen en de bloeddruk, doordat de nier de uitscheiding van water en zouten kontroleert. Recentelijk heeft men de invloed van nerveuze mechanismen op deze processen anderkend. Studies op dit terrein bespreken voor het merendeel de funktlonele invloed van de informatie die door de efferente nierzenuwen van het centrale zenuwstelsel naar de nier geleid wordt. Het feit dat de nier ook afferente zenuwezels bevat die signalen van de nier naar andere organen leiden, duidt er op dat de nier tevens een sensorfunktie heeft. In dit proefschrift wordt het konsept dat de nier als zadanig invloed heeft op mechanismen die de $1 i$ chaamsvloeistoffen en de bloeddruk reguleren, nader uitgewerkt. In de algemene inleiding (hoofdstuk 1) is deze stellingname verder onderbouwd. De funktionele betekenis van de afferente renale zenuwen (ARN) is op werschil lende manieren in ratten onderzocht. De materialen, technieken en methoden die hierbij toegepast worden, zijn beschreven in hoofdstuk 2 .

ARM aktiviteit werd elektrofysiologisch gemeten gedurende intrarenale infusles van bradykinine, adenosine en noradrenaline. Het bleek (hoofdstuk 3) dat de vuurfrekwentie van deze zenuwpopulatie toenam, wanneer deze stoffen in de renale bloedstroom toegediend werden. Dezelfde lokale infusiemethode werd gebruikt on de akute hemodynamische effekten van een woegenomen ARN-aktiviteit in wakkere raten te bestuderen. Om informatie te krijgen over de specificiteit van de opgewekte reflexen werden de resultaten vergeleken met de effekten die optraden tijdens lokale unfusie van dezelfde stoffen in een mesenteriale arterie. Voor alle geteste stoffen bleek dat de renale en mesenteriale afferenten kwalitatief eenzelfde door het sympathisch zenuwstelsel gemedleerde bloeddrukstijging veroorzakten. Dus renale en mesenteriale afferenten sluiten waarschijnlijk op dezelfde reflexbanen an en dienen eenzelfde fystologische funktie. Men neemt aan dat deze sensorische zenuwen een rol spelen in het handhaven van de organdoorbloeding. Bovendien wordt verondersteld dat een kontinue aktivering van deze reflexen bif zou kunnen dragen tot de ontwikkeling van hoge bloeddruk. Deze laatste hypothese verd getest door de hemodynamische effekten van een intrarenal bradykinine-infus gedurende langere tifd te bestuderen (hoofdstuk 5). Hoewel de bloeddruk op deze manter effektief verhoogd kon worden, zijn de infusieperioden warschijnlijk te kort geweest om blijvende veranderingen te veroorzaken in de bloeddruk. 
In hoofdstuk 6 is de invloed van ARN op de renale zoutexkretie onderzocht tijdems de overgang wan een periade van normale natriuminname naar een periode van een zeer geringe natriuminname in intakte ratten en rattem watbij de ARN door chirurgisch ingrijpen verwijderd yaren (ARN-X). De renale afferente zenuwen bleken niet noodzakelijk voor de handhaving van de natriumbalans op beide innamenivo's, maar waren in staat een in de overgangsfase kortstondige toename in de natriumexkretie te korrigeren.

Hoofdstuk 7 beschrijft de rol van ARN-afhankelijke reflexmechanismen bif kardiovastulaire aampassingen die plaatsvinden wanneer door diuretika de urineproduktie sterk wordt verhoogd. Denervatiestudies lieten zien dat ARN niet betrokken zijn bij de mechanismen die een akute toemame in de renale en latere toename in systemische valweerstand veroorzaken, wanneer furosemide en acetazollamide toegediend werden. Het mechanisme van de akute toename in de vaskulaire weerstand van de nier na toediening van deze stoffen kon niet worden opgehelderd. De later optredende algemene vasokonstriktie bleek echter renine-afhankelijk te zijn.

De invloed wan ARN op de ontwikeling van hypertenste tijdens een kontinu intrarenaal infuus van noradrenaline wordt beschreven in hoofdstuk 8. ARN $-X$ ratten vertoonden tijdens de intrarenale noradrenaline-infusies eenzelfde mate van bloeddrukstijging als intakte ratten. Het is echter mogelijk dat een aktivering van het renine-angiotensine systeem in dit model het effekt van ARN-x op de bloeddruk versluierd heef $t$.

Afferente in tegenstelling tot efferente renale zenuwen bleken niet bij te dragen tot de ontwikkeling wan hoge bloeddruk in spontaan hypertensieve ratten. De resultaten geven echter aan dat $A R N-x$ wel de ontwikkeling van de sympathische aktiviteit en de baroreflexgevoeligheid beinvloedt. Verondersteld wordt dat een disbalans tussen sensorische sympatho-excitatotre en baroreflex sympatho-inhlbitoite mechanismen zou kunnen bijdragen tot de ontwkeling van spontane hypertensie in dit model.

Hoofdstuk 10 tracht alle observaties in perspektief te plaatsen en beschrljft hoe de nier als sensor-orgaan betrokken is bij zowel fysiologische als pathofysiologsiche mechanismen die de cirkulatie reguleren. 
Aars $H_{*}$ Akre $S$. Reflex changes in sympathetic activity and arterial blood pressure evoked by afferent stimulation of renal nerve. Acta Physiol Scand $78,184-188,1970$.

Abildgaard U, Amtorp 0, Holstein-Rathlou N-H, Agerskov K, Sjontoft $\mathbb{E}$, Christensen NJ, Leyssac PP. Effect of renal venous pressure elevation on tubular sodium and water reabsorption in the dog lidiney. Acta Physiol Scand 132, 135-142, 1988.

Acara M, Roch-Ramel F, Rennick B. Bidirectional renal tubular transport of free choline: a micropuncture study. An J Physiol 236, F112-F118, 1979 .

Ammons US. Renal afferent input to thoracolumbar spinal neurons of the cat. Am $J$ Physiol 250, R435-R442, 1986.

Ammons WS. Characteristics of spinoreticular and spinothalamic neurons with renal input. J Neurobiol 58, 480-493, 1987.

Anderson WP, Selig SE, Korner PI. Role of angiotensin II in the hypertension induced by renal artery stenosis. Clin Exp Theor Pract A6 (suppl 2), $299-314,1984$.

Aström A, Crafoord J. Afferent activity recorded in the kidney nerves of rats. Acta Physiol Scand 70, 10-15, 1967.

Barajas L, Mueller J. The innervation of the juxtaglomerularapparatus and surrounding tubules: a quantitative analysis by serial section electron microscopy. J Ultrastruct Res 43, 107-132, 1973.

Barajas L; Wang $P$. Demonstration of acetylcholinesterase in the adrenergic nerves of the renal glomerular arterioles. J Ultrastruct Res 53 , $244-253,1975$.

Barajas L, Wang P. Myelinated nerves in the rat kidney. J Ultrastruct Res $65,148-162,1978$.

Barajas $L$, Wang $P$. Localization of tritiated norepinephrine in the renal arteriolar nerves. Anat Res 195, 148-162, 1979.

Barajas $\mathbb{L}$, Sokolski $K N$, Lechago $J$. Vasoactive intestinal polypeptide-immunoreactive nerves in the kidney. Neurosci Lett 43, 263-269, 1983.

Barajas $L$, Powers $K$, Mang $P$. Innervation of the renal cortical tubules: a quantitative study. Am J Physiol 247, F50-F60, 1984.

Barber JD, Moss NG. Renal chemoreceptor activation during modest reductions: in renal perfusion pressure. Kidney Int $27,187,1985$.

Barber JD, Scolrock A, Moss NG. $R_{2}$ chemoreceptor activation during renal hypoperfusion is prostaglandin dependent. Kidney Int 29, 329, 1986.

Barber JD, Harrington WW, Moss NG, Gottschalk CW. Prostaglandin blockade impairs denervation diuresis and natrduresis in the rat. Am J Phystol 250, F895-F900, 1986.

Baclow $P$, Marchbanks $\mathbb{R M}$. The effect of inhtbiting cholline dehydrogenase on choline metabolism in mice. Blochem Pharmacol 34, 3113-3122, 1985.

Beacham WS, Kunze DL. Renal receptors evoking a spinal vasomotor reflex. J Physiol (Lond) 201, 73-85, 1969.

Beers ET, Caroll RG, Young DB, Guyton AC. Effects of graded changes in reElex renal nerve activity on renal function. Am $J$ Physiol 250, $F 559$ F565, 1986.

Belcher $G$. The effects of intra-arterial bradykinin, histamine, acetylcholine and prostaglandin EI on nociceptive and non-nociceptive dorsal horn neurons of the cat. Eur J Pharmacol 56, 3835-395, 1979.

Bencsáth P, Fekete MI, Kanyicska B, Szénási G, Takacs L. Renal excretion of sodium after bilateral renal sympathectomy in the anesthet 1 zed and conscious rat. J Physiol (Lond) $331,443-450,1982$. 
Bencsáth $\mathrm{P}$, Széná⿴囗十 $\mathrm{G}$, Takács $\mathrm{L}$. Water and electrolyte transport in Henle's loop and distal tubule after renal sympathectomy in the rat. Am $J$ Physiol 249: P308-P314, 1985.

Blanchi G, Fox U, Di Francesco GF, Bardi U, Radice $M$. The hypertensive role of the kidney in spontaneously hypertensive rats. Clin Sci Mol Med 45 (supp1 1), 135-139, 1973.

Buanchi G, Pox U, Di Francesco GF, Giovanetti Am, Pagett D. Blood pressure changes produced by kidney cross transplantation between spontaneous1y hypertensive rats and normotenstve rats. Clin Sci Mol Med 47 , $435-448,1974$.

Borst JG, Borst-De Geus A. Hypertension explained by Starling's theory of circulatory homeostasis. The Lancet $677-682$, 1963 .

Bradley PB, Engel G, Fentuk $W$, Fozard JR, Humphrey PPA, Middlemiss DN, Mylecharane EJ, Richardson BP, Saxena PR. Proposals for the classification and nomenclature of funetional receptors for 5-hydroxytryptamine. Neuropharmacology 25, 563-576, 1986.

Brody MJ, Johnson AK. Role of the anteroventral third ventricle region in Eluid and electrolyte balance, arterial pressure regulation and hypertension. In: Frontiers in neuroendocrinology (eds: Martini L, Ganong WF), New York, 1980, pp 249-292.

Brooks GMCC. Newer concepts of the autonomic system's role derived from reductionist and behavioral studies of various animal species. $J$ Autonom Nerv Syst 7, 199-212, 1983.

Brooks GmcC. The autonomic nervous system and recently acquired understanding of its function and organization. In: Neurology and neurobiology, vol 31 (ed: Liss AR). New York, 1987, pp 459-463.

Buhag RD, Riley E. Chronic hypothalamic stimulation in awake rats fails to induce hypertension. Hypertension 1, 498-507, 1979.

Buñag RD, Takeda $K$, Riley $\mathrm{E}$. Spontaneous remission of hypertension in awake rats chroncially exposed to shaker stress. Hypertension 2, 311-318, 1980.

Bufag RD, Takahashi $H$. Exaggerated sympathetic responses to bradykinin in spontaneously hypertensive rats. Hypertension 3,433-440, 1981.

Burke TJ, Duchin KL. Glomerular filtration during furosemide diuresis in the dog. Kidney Int $16,672-680,1979$.

Calaresu FR, Kim P, Nakamura H, Sato A. Electrophysiological characteristics of renorenal reflexes in the cat. J Physiol (Lond) 283, 141-154, 1978.

Calaresu FR, Ciriello J. Altered concentration of catecholamines in the hypothalamus of the rat after renal denervation. Can J Physiol Pharmacol $59,1274-1277,1981$.

Calaresu FR, Ciriello J. Renal afferent nerwes affect discharge rate of medullary and hypothalamic single units in the cat. J Autonom Nerv Syst $3,311-320,1981$.

Calaresu FR. Autonomic preganglionic neurons - the final common pathway of physiological regulations. J Autonom Nerv Syst 5, 3-7, 1982.

Caverson MM, Ciriello J. Effect of stimulation of afferent renal nerves on plasma levels of vasopressin. Am J Physiol 252, R801-R807, 1987.

Caverson MM, Ciriello $J$. Contribution of paraventricular nucleus to afferent renal nerve pressor response. Am J Physiol 254, R531-R543, 1988. Chiuch CC, Kopin IJ. Hyperresponsitivity of spontaneously hypertensive rats
to indirect measurement of blood pressure. Am J Physiol 234, H690H695, 1978.

Christensen $S$, Steiness $E$, Christensen H. Tubular sites of furosemide natrifuresis in volume replaced and volume depleted conscious rats. J Pharmacol Exp Ther 239, 211-218, 1986.

Chuang EL, Relneck HJ, Osgood RW, Kunau RT, Stein JH. Studies on the mecha- 
nism of reduced urinary osmolarity after exposure to the renal papilla. J Clin Invest $61,633-639 ; 1978$.

Ciriello J, Calaresu $F R$. Hypothalamic projections of renal afferent nerves in the cat. Can J Physiol Pharmacol 58, 574-576, 1980.

Ciriello $J$, Calaresu. FR. Vagal innervation of the kidney in the rat. Can Fed Biol Soc 25, 83, 1982.

Ciriello $J$, Calaresu FR. Central projections of afferent renal fibers in the rat: an anterograde transport study of horseradish peroxidase. I Autonom Nervous Syst $8,273-285,1983$.

Cirifello $J$, Kline RL, zhong $T-X$, Caverson M. Lesions of the paraventricular nucleus alter the development of spontaneous hypertension in the rat. Brain Res 310, 355-359, 1984.

Ciriello J, Caverson MM. Central organization of afferent renal nerve pathways. Clin Exp Hypert Theor Pract A9 (Suppl 1), 33-46, 1987.

Cody RJ, Rodger RF, Hartley LH, Burton J, Herd A. Acute hypertension in a non-human primate: humoral and hemodynamic mechanisms. Hypertension 4, $219-225,1982$.

Coggeshall RE. Law of separation of function of the spinal roots. Physiol Rev 60, 716-756, 1980 .

Colleridge HM, Coleridge JCG, Kaufman MP, Dangel A. Operational sensitivity and acute resetting of aortic baroreceptors in dogs. Circ Res 48 , $676-684,1981$.

Coote Jh, Sato $Y$. Reflex regulation of sympathetic nerve activity in the spontaneously hypertensive rat. Circ Res $40,571-577,1977$.

Cowley Jr AV, Lohmeier TE. Changes in renal vascular sensitivity and arterial pressure associated with sodium intake during long-term intraw renal norepinephrine infusions in dogs. Hypertension $1,549-558$, 1979.

Cuche JL, Liard JF. Tissue catecholamines following renal denervation in spontaneously hypertensive rats. Experientia 37, 1089-1090, 1981.

Dahl LK, Heine M. Thompson K. Genetic influence of the kidney on blood pressure. Evidence for chronic renal homografts in rats with opposite predispositions of hypertension. Circ Res 34, 94-102, 1974.

Davies DL, Wilson GM. Diuretics. Mechanisms of action and clinical application. Drugs 9: $178-226,1975$.

Davis Jo, Freeman RH. Mechanisms regulating renin release. Physiol Rev 56, $1-56,1976$.

Dector DL, Weems WA. A study of renal-efferent neurones and their neural connections with cat renal ganglia using intracellular electrodes. $\mathrm{J}$ Physiol 321, 611-626, 1981.

De Leeuw PU, DeBos R, Van ES PN, Birkenbager WH. Effect of sympethetic stimulation and intrarenal alpha-blockade on the secretion of renin by the human idiney. Eur J Clin invest 15, 166-170, 1985.

De Leeuw $P W$, Birkenhäger. Efferent renal nerwe activity in hypertensive man. Clin Exp Fypert Theor Pract A9 (supp1 1), 281-292, 1987.

Deth RC, Payme RA, Peecher DM. Influence of furosemide on Rubidium-86 up take and alpha-adrenergic responsiveness of arterial smooth muscle. Blood Vessels 24, 321-333, 1987 .

DeWolf WC, Fraley EE. Renal pain. Urology, 6, 403-408, 1975.

DiBona GF. The functions of the renal nerves. Rev Physiol. Biochem Pharmacol $94,75-181,1982$.

DiBona GF, Sawin LL. Role of renal alpha-2 adrenergic receptors in spontan eously hypertensive rats. Hypertension $9,41-148,1987$.

DiBona GF. Mineral Electrol Metab 1988, in press.

Dikshit K, Vyden JK, Forrester JS, Chatterjee K, Prakas R, Swan HJC. Renal. and extrarenal hemodynamic effects of furosemide in congestive heart failure after myocardial infarction. N Engl J hed 288, 1987-1090, 
1973.

Diz DI, Najlett $A$, Baer PG. Renal denervation at weaning retards development of hypertension in New Zealand genetically hypertensive rats. Hypertension $4,361-368,1982$.

Donovari MK, Wyss JM, Winternutz SR. Localization of renal sensory neurons using the fluorescent dye technique. Brain Res 259, 119-122, 1983.

Drukker $J$, Groen $G J$, Boekelaar $A B$, Baljet $B$. The extrinsic innervation of the rat kidney. Clin Exp Hypert Theor Pract A9 (suppl 1), 15-32, 1987.

Dzlelak DJ, Norman RA. Renal nerves are not necessary for onset or maintenance of DOCA-salt hypertension in rats. An $J$ Physiol 249, H945-H949, 1985 .

Esler $M$, Jennings $G$, Korner $P$, Willett $I_{*}$. Dudley $F$, Hasking $G$, Anderson $W$, Lambert G. Assessment of human sympathetic nervous system activity from measurements of norepinephrine turnover. Hypertension $11,3-21$, 1988.

Evenwel $\mathbb{R T}$, Kasbergen CM, Struyker-Boudier HAJ. Central and regional hemodynamics and plasma volume distribution during the development of spontaneous hypertension in rats. Clin Exp Hypert A5, 1511-1536, 1983.

Paber JE, Brody MJ. Afferent renal nerve dependent hypertension following acute renal artery stenosis in the conscious rat. Circ Res 57, 676$688,1985$.

Faber JE. Role of prostaglandins and kinins in the renal pressor reflex. Hypertension $10,522-532,1987$.

Faber JE. Inhibitory effect of the contralateral kidney on the renal pressor reflex evoked by ipsilateral renal artery stenosis. Clin Exp Theor Pract A9 (suppl 1), $241-258,1987$.

Faber JE, Gettes DR. Renal pressor reflex: involwement of sympathetic vasoconstrictor mechanisms. Am J Physiol 252, H1147-H1158, 1987.

Fasth $S$, Hedlun $H$, Hulthén $L$, Nordgren $S$, öresland $T$. The effects of 5-hydroxytryptamine on large intestinal motility and blood flow in the cat. Acta Physiol Scand 118, 329-336, 1983.

Felder RB. Excitatory and inhibitory interactions anong renal and cardiovascular afferent nerves in dorsomedial medulla. Am J Physiol 250 , R580-R588, 1986.

Fernăndez-Repoliet E, Silva-Netto $\mathrm{CR}$, Colindres RE, Gottschalk CW. Role of renal nerves in maintaining sodium balance in unrestrained conscious rats. Am J Physiol 249, F819-F826, 1985.

Fink GD, Brody MJ. Neurogenic control of the renal circulation in hypertension. Fed Proc 37, 1202-1208, 1978.

PInk GD, Brody MJ. Impaired neurogenic control of renal vasculature in renal hypertensive rats. Am J Physiol 238, H770-H775, 1980.

Folkow B, Rubenstein EH. Cardiovascular effects of acute and chronic stimulation of the hypothalamic defence area in the rat. Acta Physiol Scand $68,48-57,1966$.

Folkow B. Physlological aspects of primary hypertension. Physiol Rev 62, $347-504,1982$.

Francisco LI, Sawin LL, DiBona GF. Renal sympathetic nerve activity and the exaggerated natriuresis of the spontaneously hypertensive rat. Hypertension 3, 134-138, 1981 .

Fujita T, Sato $Y$. Changes in renal and central noradrenergic activity with potassitum in DOCA-salt rats. Am J Physiol 246, F670-F675, 1984.

Fukuda $Y$, Sato A, Trzebskil A. Carotid chemoreceptor discharge responses to hypoxia and hypercapnia in normotensive and spontaneously hypertensive rats. J Autonom Nerv Syst 19, 1-11, 1987. 
Ganten D, Lange RE, Lehmann B, Unger T. Brain anglotensin: on the way to becoming a well-studied neuropeptide system. Blochem Pharmacol 33 , $3523-3528,1984$.

Gattone VH II, Marfurt CF, Dallie S. Extrinsic imervation of the rat kidney: a retrograde study. Am J Physiol 250, F189-F196, 1986.

Genest J. Personal views on the mechanisms of essential hypertension. In: Hypertension: physiopathology and treatment (eds: Genest J, Kuchel 0 , Hamet P, Cantin M). McGraw-Hill Book Company, New York, 1983, pp $599-675$.

Gerber JG. Role of prostaglandins in the hemodynamic and tubular effects of furosemide. Fed Proc 42, 1707-1710, 1983.

Gerkens JF. Does furosenide have vasodilator activity? Trends Pharmacol Sci. $8,254-257,1987$.

Gershon MD, Erde SM. The nervous system of the gut. Gastroenterology 80 , $1571-1594,1981$.

Gilmare JP, Tomomatsu E. Renal mechanoreceptors in non-human primates. Am J Physiol 248, R202-R207, 1985.

Goldblatt H, Lynch J, Hanze RF, Summerville WW. The production of persistent elevation of systolic blood pressure by means of renal ischernia. $\mathrm{J}$ Exp Med 59, 347-379, 1934.

Golin R, Genovesi S, Stella A, Zanchetti A. Afferent pathways of neural reno-renal. reflexes controlling sodium and water excretion in the cat. J Hypertension 5, 417-424, 1987.

Gootman PM, Cohen MI, DiRusso SM, Sica AL, Cohen HL, Eberle LP, Rudell AP, Gootman $\mathrm{M}$. Periodicities in spontaneous preganglionic sympathetic discharge. In: Organization of the autonomic nervous system: central and peripheral mechanisms (eds: Cirello J, Calaresu FR, Renaud LP, Polosa C). Liss AR Inc., New York, 1987, pp 133-142.

Gosling JA. Observations on the distribution of intrarenal nervous tissue. Anat Rec $163,81-88,1969$.

Gosling JA. "The innervation of the upper urinary tract. J Anat 106, 51-61, 1970.

Gottschalk CN, Moss $\mathrm{NG}$, Colindres RE. Neural control of renal function in health and disease. In: The kidney: physlology and pathophysiolgoy (eds: Seldin DW, Giebisch G). Raven Press, New York, 1985, pp 581611 .

Guidi E, Bianchi G, Dallosta V, Cantaluppi A, Mandelll V, Vallino F, Polli E. Influence of familial hypertension of the donor on the blood pressure and antihypertensive therapy of kidney graft recipients. Nephron $30,318-323,1982$.

Guyton AC, Coleman TG, Cowley Jr AW, Manning Jr RD, Norman RA, Ferguson JD. A systems analysis approach to understanding long-range arterial. blood pressure control and hypertension. Cire Res 35, 159-176, 1974 .

Guyton AC (ed). Textbook of medical physiology. WB Saunders Company, Philadelphia, 1986.

Guyton AC, Manning RD, Norman RA, Montani J-P, Lohmeier TE, Hall JE. Current concepts and perspectives of renal vollume regulation in relationship to hypertension. J Hypert 4 (supp1 4), 49-56, 1986.

Guzman F, Braun C, Lim RKS. Visceral pain and the pseudaffective response to intra-arterial injection of bradykinin and other algesic agents. Arch Int Pharmacodym Ther 136, 353-384, 1962.

Hageman GR, Gantenberg NS, Katholi RE. Differential activation of sympathetic efferent activities during intrarenal adenosine infusion in the dog. Fed Proc 46, 1238, 1987.

Harris H, Harris $\mathbb{R}$. Renal sympatheticotonus, renal paln and renal. sympathectomy. Br J Urol $2,367-3374,1930$.

Haywood JR, Schaffer RA, Fastenow C, Fink GD, Brody MJ. Reglonal blood flow 
measurement wh pulsed Doppler flow meter in conscious rats. Am I Physiol 241, H273-H278, 1981.

Hallback $K$. Consequence of social isolation on blood pressure, cardiowascular reactirity, and design in spontaneously hypertensive rats. Acta Physiol Scand 93, 455-465, 1975.

Hebert SC, Andreoll TE. Control of NaC1 transport in the thick ascending 14mb. Am J Physiol 246, F745-F756, 1984 .

Hermansson $K, 0 j t e g$, Wolgast $M$. The renorenal reflex: evaluation from renal blood flow measurements. Acta Physiol Scand 120, 207-215, 1984.

Heusch $G$, Deussen $A$, Thämer $V$. Cardiac sympathetic nerve activity and progressive vasoconstriction distal to coronary stenoses: feedback aggravation of myocardial ischemia. J Autonom Nerw System 13, 311 $326,1985$.

Hinshaw LB, Day SB, Carlson CH. Tissue pressure as a causal factor in the autoregulation of blood flow in the isolated perfused kidney. Am $J$ Physiol $197,309-317,1959$.

Hjemdahl P. Physiological aspects on catecholamine sampling tife $S c 141$, $841-84.4,1987$.

Högestät Ed, Johansson 0 , Andersson K-E, Kullendorff $C-M$. Influence of renal. denervation on vascular responsiveness of isolated rat intrarenal arteries. Acta Physiol Scand 132, 59-66, 1987.

Imbs JL, DeSaulles E, Schwartz J. Action du chlorure de sodium hypertonique et du furosemide, sur le flot medullaire: role de la rë́nine. J Pharmacol (Paris) $1,487-500,1970$.

Janssen BJA, Struyker-Boudier HAJ, Smits JFM. Dorsal spinal rhizotomy of renal afferent nerves prevents the reflex effects induced by intrarenal bradykinin infusion in the rat. Pharmaceut Wkbl Sci Ed 7, 231, 1985 .

Janssen BJA, Debets JJ, Struyker-Boudier HAJ, Smits JFH. Role of sensory renal nerves in the development of spontaneous hypertension. Clin Exp Hypert Theor Pract A9 (supp1 1), 227-239, 1987.

Janssen BJA, Struyker-Boudier HAJ, Smits JFM. Do afferent renal nerves play an important role in the development of spontaneous hypertension in rats? J Hypertension 5. (suppl 5), 375-377, 1987.

Janssen BJA, Smits JFM. Renal nerves in hypertension. Mineral Electrol Metab, in press, 1988.

Janssen BJA, Van Essen H, Vervoort-Peters HTM, Thijssen HHW, StruykerBoudier HAJ, Smits JFM. Effects of renal sensory denervation on sympathetic function and baroreflex sensitivity in spontaneously hypertensive rats. Submitted for publication.

Janssen BJA, Van Essen H, Vervoort-Peters HTM, Thijssen HHW, Derkx FHM, struyker-Boudier HAJ, Smits JFM. Effects of complete renal denervation and selective afferent renal denervation on the hypertension induced by intrarenal noreplnephrine infusion in conscious rats. Submitted for publication.

Jänig Wplnal cord integration of visceral sensory systems and sympathet1c nervous system reflexes. Prog Brain Res 67, 255-277, 1986.

Jänig W. Pre- and postganglionic vasoconstrictor neturons: differentiation, types and discharge properties. Ann Rev Physiol 50, 525-539, 1988.

Johnston GD, Nicholls DP, Kondowe GB, Finch MB. Comparison of the acute vascular effects of furosemide and bumetanide. $\mathrm{Br} J \mathrm{Cl}$ in Pharmacol $21,359-364,1986$.

Judy WV, Watanabe AM, Henry DP, Besch HR, Murphy WR, Hockel GM. Sympathetic nerve activity: role in regulation of blood pressure in the spontaneously hypertensive rat. Circ Res 38 (suppl 2), 21-29, 1976.

Jullus S, Sanchez R, Brant D. Pressure increase to external hindquarter 
compression in dogs: a facultative regulatory response. J Hypert 4 (suppl 6), 54-56, 1986.

Julius $S$. The blood pressure seeking properties of the central nervous system. J Hypertension $6: 177-185,1988$.

Julius S, Brant D. Fallure to develop established hypertension with repeated neurogenic pressor episodes. Abstract 881,12 th Scientific Meeting International Society of Hypertenison, Kyoto, Japan, 1988.

Katholi RE, Carey RM, Ayers CR, Vaughan ED, Yancey MR, Morton C1. Production of sustained hypertension by chronic intrarenal norepinephrine infusions in conscious dogs. Circ Res 40 (suppl 1), 118-126, 1977.

Katholi $R E$, Naftilan $A$, Oparil $S$. Importance of renal sympathetic tone in the development of DoCA-salt hypertension in the rat. Hypertension 2 , $266-273,1980$.

Katholi RE, Winternitz SR, Oparil S. Role of the renal nerwes in the pathogenesis of one-kidney renal hypertension in the rat. Hypertension 3 , $404-409,1981$.

Katholi RE, Whitlow PL, Winternitz SR, Oparil S. Importance of the renal nerves in the established two-kidney, one-clip Goldblatt hypertension in the rat. Hypertension 4 (suppl 2), 166-174, 1982 .

Katholi RE, Winternitz SR, oparil S. Decrease in peripheral sympathetic nervous system activity following renal denervation or unclipping in the one-kidney, one-clip Goldblatt hypertensive rat. J Clin Invest $69,55-62,1982$.

Katholi RE. Renal nerwes in the pathogenesis of hypertension in experimental aninals and humans. Am J Physiol 245, F1-F14, 1983.

Katholi RE, Hageman GR, Whitlow PL, Woods WT. Hemodynamic and afferent renal nerve responses to intrarenal adenosine in the dog. Hypertension 5 (suppl 1), 149-153, 1983.

Katholi RE. Renal nerves and hypertension: an update. Fed Proc 44, 2846$2850,1985$.

Katholi RE, McCann WP, Woods WT. Intracenal adenosine produces hypertension wa renal nerves in the one-kidney, one-clip rat. Hypertension 7 (suppl 1), 88-93, 1985 .

Katholi RE, Noods WT. Afferent renal nerves and hypertension. Clin Exp Hypert Theor Pract A9 (supp1 1), 211-226, 1987.

Kawabe KT, Watanabe TX, Shiono K, Sokabe $\mathrm{H}$. Influence on blood pressure of renal isografts between spontaneously hypertensive and normotensvie rats, utilizing the F1 hybrids. Jpn Heart J 19, 886-894, 1978.

Kiernar $J A$. Hypothesis concerned with axonal regeneration in the mammalian mervous system. Biol Rev 54, 155-197, 1979.

Kleinjans JCS. Stimulation of renal adrenergic mechanisms as a model. for the development of hypertension. PhD thesis, University of Limburg. Maastricht, 1983.

Kleinjans JCS, Smits JFM, Kasbergen CM, Vervoort-Peters HTM, StruykerBoudier HAJ. Blood pressure response to chronic low-dose intrarenal. noradrenaline infusion in conscious rats. Clin Sci 65, 111-116, 1983.

Kleinjans JCS, Smits JFM, Kasbergen CM, Struyker-Boudier HAJ. Body fluid and salt homeostasis during hypertension caused by chronlc intrarenal norepinephrine infusions $\mathbb{i n}$ conscious rats. J Hypertension $\mathbb{1}$ (suppl 2), 207-209, 1983 .

Kleinjans JCS, Smits JFM, Kasbergen CM, Van Essen H, Struyker-Boudier HAJ. Evaluation of renal function during intrarenal norepinephrine infusions in conscious rats. Renal Phys101 7, 243-250, $1984 \mathrm{a}$.

Kleinjans JCS, Smits JPM, Van Essen H. Kasbergen CM. Struyker-Boudler HAJ. Hemodynamic characterization of hypertension induced by chronic intrarenal intravenous infusion of norepinephrine in conscious rats. Hypertension $6,689-699,198.4 \mathrm{~b}$. 
Kine RL, Kelton PM, Mercer PF. Effect of renal denervation on the development of hypertension in spontaneously hypertensive rats. Can $J$ Physlol Pharmacol 56,818 .822, 1978.

Kline $\mathrm{KL}$, Metcer PF. Functional reinnerwtion and development of supersensitivity to NE after renal denerwation in rats. Am $J$ Physiol 238 , R353-R358, 1980.

Kllne RL, Strant PJ, Mercer PF. Effect of renal denervation on arterial pressure and renal norepinephrine concentrations in Vistar-Kyoto and spontaneously hypertensive rats. Can $J$ Physiol Fharmacol 58, 13841388,1980 .

KIIne RL. Reduced renal vascular response to nerve stimulation and norepinephrine in DoCA-salt hypertensive rats. Can J Physiol Pharmacol 61, $464-471,1982$.

K1ine RL, Patel KP, Mercer PF. Evidence that afferent renal nerwes (ARN) are not involved in the effect of renal denervation in spontaneously hypertensive rats (SHR). Fed Proc 42,743, 1982.

KIine RL, Patel KP, Ciriello J, Mercer PF. Effect of renal denervation on arterial pressure in rats with aortic nerve transsection. Hypertenston $5,468-475,1983$.

Kline RL, Denton KM, Anderson WR. Effect of renal denervation on the development of cellophane-wrap hypertension in rabbits. Clin Exp Hypert Theor Pract $A B, 1327-1342,1986$.

Knox FG, Granger JP. Control of sodium excretion: the kidney produces under pressure. NIPS 2, 26-29, 1987.

Knuepfer Mm, Gebhart GF, Brody MJ. Effects of baroreceptor activation on single unit activity of the anteroventral third ventricle region of the rat. Neurosci Letters $56,79-85,1985$.

Knwepfer MM, Schram LP. Properties of renobulbar afferent fibers in rats. An J Physiol 248, R113-F119, 1985.

Koepke JP, Jones $\$$, DiBona GF. Renal merve activity and renal function during environmental stress in DoCA-NaCl rats. Am I Physiol 251, $\mathrm{R} 289-\mathrm{R} 294,1986$.

Kopp UC, Bradley T, Hjemiahl. P. Renal venous outflow and urinary excretion of norepinephrine, epinephrine and dopamine during graded renal neve stimulation. Am J Physio1 244, E52-E60, 1983.

Kopp UC. Renorenal reflexes: neural and functional responses. Fed Proc 44, $2834-2839,1985$.

Kopp UC, Smith LA, DiBona GF. Renorenal reflexes: neural components of ipsilateral and contralateral renal responses. Am J Physiol 249, F507-F517, 1.985.

Kopp UC, Smith LA, DIBona GF. Impaired renorenal reflexes in spontaneonsly hypertensive rats. Hypertension 9, 69-75, 1987.

Kopp UC, Buckley-Bleiler Rl. Renorenal reflexes impaire in 2-kidney, 1-clip renal hypertensive rats. FASEB Journall A1717, abstr 8253, 1988.

Kotchen "TA, Velch WJ, Lorenz JN, Ott CE. Renal tubular chloride and renin release. J Lab Clin Med $110,533-540,1987$.

Kottke Fu, Kubicek WG, Visscher MB. The production of arterial hypertension by chronde renal artery nerve stimulation. Am J Physiol 145, 38-47, 1945.

Krayaclch $J$, Kline RL, Mercer PF. Supersensitivity to NE alters renall function of chronically denervated rat kidneys. Am J Physiol 252, F856-F864, 1987.

Krieger $\mathbb{C M}$. Neurogenic mechanisms in hypertension: resetting of the baroreceptors. Hypertension 8 (suppl 1), 7-14, 1986.

Krueger AD, Lee JY, Yang P-C; Papaioannou SE, Walsh GM. Selective vasodilatation produced by renal denervation in adult spontaneously hypertensive rats. Hypertension $8,372-378,1986$.

Kunze DL, Rapid resetting of the carotid baroreceptor reflex in the cat. 
Am J Physiol 241, H802-H806, 1981.

Kuo DC, Nadelhaft I, Hisamitsu T, De Groat WC. Segmental distribution and central projections of renal afferent fibers in the $c$ at studied by transganglionic transport of horseradish peroxldase. J Comp Neurol 216, $162-174,1983$.

Rvetnansky $R$, McCarty $R$, Thoa NB, Lake CR, Ropin IJ. Sympatho-adrenal responses in spontaneously hypertensive rats to immobilization stress. Am J Physiol 236, $\mathbb{1 4 5 7 - H 4 6 2 , ~} 1979$.

Lais LT, Bhantagar RR, Brody MJ. Inhibition by dark adaptation of the propgress of hypertension in the spontaneously hypertensive rats. Circ Res 34 and 35 (suppl 1), 55-66, 1974.

Lappe RW, Henry DP, Willis LR. Contribution of renal sympathetic nerves to the urinary excretion of norepinephrine. Can $J$ physiol Pharmacol 60 , $1067-1072,1982$.

Lappe RW, Webb RL, Boutelle S, Brody MJ. Identification of central projections of renal afferent nerves. Fed Proc 41, 1258, 1982.

Lappe RW, Webb RL, Brody MJ. Selective destruction of renal afferent versus efferent nerves in rats. Am Physiol 249, R634-R637, 1985.

Laughton $\mathrm{J}$. The function of the sympathetic nerve supply to the kidney and the effect of denervation. Br J Clin Pract 26, 353-357, 1972.

Lee JY, Walsh GM. Systemic and regional hemodynamic effects of renall denerwation in spontaneously hypertensive rats. J Hypertension 1, 381-386, 1983.

LeNoble LML, Lappe RW, Brody MJ, Struyker-Boudier HAJ, Smits JFM. Selective efferent chemical sympathectomy of rat kidneys. Am $J$ Hhysiol 249 , R496-R501, 1985.

Lew WY, Longhurst JC. Substance $P, 5$-hydroxytryptamine and bradykinin stimulate abdominal visceral afferents. Am J Physiol 250, R465-R567, 1987.

Liard JF. Renal denervation delays blood pressure increase in the spontaneously hypertensive rats. Experientia $33,339-340,1977$.

Lifschitz MD. Lack of a role for the renal nerves in renal sodilum reabsorp tion in conscious dogs. Clin Sci Mol Med 54, 567-572, 1978.

Liu CN, Chambas Wh. Intraspinal sprouting of dorsal root axons. Arch Neurol Psychiat 79, 46-61, 1958 .

Lohmeier TE, Tillman LJ, Carroll RG, Brown AJ, Guyton AC. Malignant hypertensive crisis induced by chronic intrarenal norepinephrine infusion. Hypertension 6 (supp1 1), 177-182, 1984.

Longhurst JC, Dittman LE. Hypoxia, bradykinin and prostaglandins stimulate ischemically sensitive visceral afferents. An J Physiol 253, H556H567, 1987 .

Longhurst JC, Kaufman MP, Ordway GA, Musch TT. Effects of bradykinin and capsaicin on endings of afferent fibers from abdominal visceral organs. Ant J Physiol 247, R552-R559, 1984.

Ludens JH, Heitz DC, Brody MJ, Williamson HE. Enhancenent of renal blood flow by furosemide. I Pharmacol Exp Ther 163, 456-460, 1968.

Ludens $J H$, Hook $J B$, Brody MJ. Differential effect of furosentide on renal and limb blood flows in the conscious dog. J Pharmacol Exp Ther 17l, $300-306,1970$.

Lundin $S$, Ricksten SE, Thorén P. Renal sympathetic activity in spontaneous$1 y$ hypertensive rats and normotensive controls, as studied by three different methods. Acta Physiol Scand 120, 265-272, 1984.

Malliani A. Cardiovascular sympathetic afferent fibers. Rev Physiol Biochem Pharmacol $94,1-74,1982$.

Mathias CJ, Kooner JS, Peart S. Neurogenic components of bypertension in human renal artery stenosis. Clin Exp Hypert. Theor Pract A9 (suppl. 
1). $293-306,1987$.

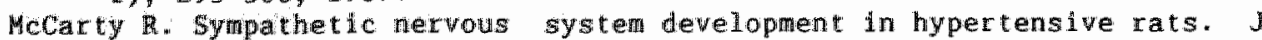
Hypertension: 4 (suppl 3), 155 157, 1986 .

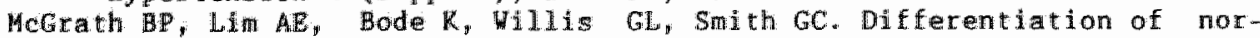
adrenergic and dopaminergic nerves in the rat kidney: evidence ageinst signiflcant dopaninergic innervation. Clin Exp Pharmacol Physiol 10, 543-553, 1983.

Meckler RI, Weaver LC. Characteristics of ongoing and reflex discharge of single renal and splenic sympathetic postganglionic fibers in cats. J Physiol (Lond) $396,139-153,1988$.

Hiler RJ. Bradykinin highlights the role of phospholipid metabolism in the control of nerve excltability. TINS 10,226-228, 1987.

$141 . \mathrm{s} \mathrm{E}$, Smith PG. Catecholamine infusions can increase sympathetic nerve activity reflexly by stimulating arterial chemoreceptors. Fed Proc $42,895,1983$.

Mitcheil GAG. The nerve supply of the kidney. Acta Anat 10, 1-37, 1950.

Milielle HL, Hall JE, Woods LL, Montani JP, Dzielak. DJ, Pan $Y-J$. Role of renal nerwes in compensatory adaptation to chronic reductions in sodium intake. Am J Physiol 252, F291-F298, 1987.

Moss NG. Renal function and renal afferent and efferent nerve activity. Am J Phys 101 243, F425-F433, 1982.

Moss NG. Electrophysiology of afferent renal nerves. Fed Proc 44, 28282833,1985 .

Moss NG, Powell SL, Falk RJ. Intravenous cyclosporine activates afferent and efferent renal nerves and causes sodium retention in innervated kidneys in rats. Proc Nat Acad Sci USA 82, 8222-8226, 1985.

Moss NG. Electrophysiological characteristics of sensory mechanisms in the kidney. Clin Exp Hypert Theor Pract A9 (suppl 1), 1-14, 1987.

Nagaoka A, Kakihana M. Effects of renal sympathectomy on sodium and water excretion in stroke-prone hypertensive rats. Jpn J Pharmacol 32 , $591-597,1982$.

Nash FD, Selkurt EE. Effects of elevated ureteral pressure on renal blood flow. Circ Res 15 (suppl 142), 1964.

Nievelstein HMNW, Schaefer MAG, Struyker Boudier HAJ, Smits JPM. Effect of baroreflex activation on regional hemodynamics in conscious normoten-sive rats. J Hypertension 4 (suppl 6), 287-289, 1986.

Niljima A. Afferent discharges from arterial mechanoreceptors in the kidney of the rabbit. J Physiol (Lond) 219, 477-485, 1971.

Nijima $A$. The effect of efferent discharges in renal nerves on the activity of arterial mechanoreceptors in the kidney in rabbit. $J$ Physiol (Lond) $222,335-343,1972$.

Nidjima A. Studies on the blood pressure sensitiwe receptors in the rabbit kidey in vivo. Jpn J Physiol 22, 433-440, 1972.

Nijima A. observation on the Localizatlon of mechanoreceptors in the kidney and afferent nerve flbers in the cenal nerves in the rabbit. J Physiol (Lond) $245,81-90,1975$.

Nifjma A. Glucose-sensitive afferent nerve fibers in the liver and their role in food intake and blood glucose regulation. J Autonom Merv Syst 9: $207-220,1983$.

Norman RA, Collemen TG, Dent AC. Continuous monitoring of arterial pressure indicates sino-aortlo denervated rats are not hypertensive. Hypertension 3, 119-125, 1981.

Norman RA, Dzielak DJ. Role of renal merves in onset and maintenance of spontaneous hypertension. An $J$ Physiol 243, H284-H288, 1982.

Norman $R A$. Role of the renal nerves in one-kidney, one-clip hypertension in rats. Hypertension $6,622-626,1984$.

Norvell JE, Anderson JE. Assessment of possible parasympathetic innervation 
of the kidney. J Autonom Nerw System 8, 291-294, 1983.

Notley RG. Electron microscopy of the upper ureter and the pelw-ureter junction. Br J Urol 40,37-52, 1968 .

Ohno T, Yajima T, Urano T, Nakamuro K. Interaction of prostaglandin $2_{2}$ and bradykinin in the induction of afferent splanchic nerve discharges in cats. Jpn J Pharmacol 34, 191-202, 1983.

okamoto $\mathbb{K}$, Aoki $\mathrm{K}$. Development of strain of spontaneousiy hypertensive rats. Jpn Circ J 27, 282-293, 1963.

oliver JA, Pinto J, Sciacca Rr, Cannon PJ. Basal norepinephrine overflow into the renal vein: effect of renal nerve stimulation. Am J Physiol 239, F371-F377, 1980 .

oparil $\mathrm{S}$. The sympathetic nervous system in clinical and experimental hypertension. Kidney Int 30, 437-452, 1986.

ordway GA, Boheler KR, Longhurst, JC. Stimulating intestinal afferents reflexly activates cardiowascular system in cats. Am J Physiol 254, H354-H360, 1988.

Ordway GA, Longhurst JC. Cardiovascular reflexes arising from the gall bladder of the cat: effects of capsaicin, bradykinin and distension. Circ Res 52, 26-35, 1983.

Ordway GA, Longhurst JC, Mitchell JH. Stimulation of pancreatic afferents reflexly activates the cardiovascular system in cats. Am J Physiol 245, R820 $-R 826,1983$.

Osborn JL, Kinstetter DD. Effects of altered $\mathrm{NaCl}$ intake on renal hemodymamic and remin release responses to RNS. Am J Physiol 253, F976-F981, 1987.

osswald $H$. The role of adenosine in the regulation of glomerular filtration rate and renin secretion. Trends Pharmacol Sci, March, 94-97, 1984.

Osterziel $\mathrm{KF}$, Julius $\mathrm{S}$, Brant $\mathrm{D}$. Blood pressure elevation during hindquarter compression in dog is neurogenic. J Hypert $4,411-417,1984$.

Pagani M, Pizzinzelli P, Furlan R, Guzetti S, Rimoldi 0, Sandrone G, Malliani A. Analysis of the pressor sympathetic reflex produced by tintracoronary injections of bradykinin in conscious dogs. Circ Res 56 , $175-183,1985$.

Palkovits M, Zaborsky L. Neuroanatomy of central cardiovascular control. Nucleus tractus solftarii: afferent and efferent neuronal connections in relation to the baroreceptor reflex arc. Prog Brain Res 47, 9-34, 1977.

Pan J-Y, Bishop VS, Ball NA, Haywood JR. Inability of dorsal spilnal rhizotomy to prevent renal wrap hypertension in rats. Hypertenton 7 , $722-728,1985$.

Pappenheim S. Arch Anat Physiol Wissensch Med 7, 543, 1840. In: Baker R, Tarabulcy E. Effects of contralateral nephrectomy and renal nerves on renal function. Med Ann Distr Columbia 35, 467-469, 1966.

Persson AEG, Vright FS. Evidence for feedback medlated reduction of glomerular filtration rate during infusion of acetazolamide. Acta physiol Scand $114,1-7,1982$.

Pettinger WA, Sanchez A, Saavedra J, Haywood JR, Gandler T, Rodes T. Al.tered cenal alpha-2 adrenergic receptor regulation in genetically hypertensive rats. Hypertension 4 (supp1 2), 188-192, 1982 .

Pines YL. The electrophysiological characteristics of the aferent connections of the kidney with the central nervous system. Sechenov Physiol J USSR $46,1622-1630,1960$.

Protasoni G, Genovesi S, Assi C, Stella A, Zanchetti. A. Meural control of renal excretory functions in normotensive and spontaneously hyperten sive rats. J Hypert 5 (suppl 5), 365-366, 1987. 
Recordari GM, Moss NG, Waselikov L. Renal chemoreceptors in the rat. Circ Res $43,534-543,1978$.

Recordat 1 GM, Hoss MG, Genovesi $S$, Rogenes PR. Renal receptors in the rat sensitive to chemical alterations of their environment. Circ Res 46 , $395-405,1980$.

Regoll D, Barabé $J$. Pharmacology of bradykinin and rellated kinins. Pharnacol Rev 32, 1-46, 1980.

Rettig $x$, Stauss H, Folberth $C$, Unger T. Transplantation of kidneys from spontaneously hypertensive rats induces hypertension in normotensive recipients. Proceedings 12 th Scientific Meeting International Society of Hypertension, Kyoto, Japan, 1988 , abstr nr 138.

Robie N. Presynaptic inhibition of canine renal adrenergic nerves by acetylcholine in vivo. Am J Physiol 237, H326-H331, 1979.

Rogenes PR, Gottschalk CW. Renal function in conscious rats with chronic unilateral renal denervation. Am J Physiol 242, F140-F148, 1982.

Rogenes PR. Single-unit and multiunit analyses of renorenal reflexes elicited by stimulation of renal chemoreceptors in the rat. I Autonom Nerv Sys $6,143-156,1982$.

Ross CA, Ruggiero DA, Park DH, Joh TH, Sved AF, Fernandez-Pardal J, Saavedra JM, Reis DJ. Tonic vasomotor control by the rostral ventrolateral medulla: effect of electrical or chemical stimulation of the area containing $C 1$ adrenaline neurons on arterial pressure, heart rate, and plasma catecholamines and vasopressin. J Neurosci 4, 47.4-494, 1984 .

Sanchez A, Pettinger HA. Dietary sodium regulation of blood pressure and renal alpha-1 and alpha-2 receptors in WKY and SH rats. Life Sc1 29 , $2795-2802,1981$.

Saphir R, Rapaport E. Cardiovascular responses of the cat to mesenteric intra-arterial admintration of nicotine, cyanide and venous blood. Circ Res 25, 713-724, 1969.

Sato A, Schmidt RE. The modulation of visceral function by somatic afferent activity. Jpn J Physiol 37, 1-17, 1987.

Säynäwälammi $\mathrm{P}$, Vaalasti $\mathrm{A}$, Pyykönen $\mathrm{M}-\mathrm{L}$, Ylitalo $\mathrm{P}$, Vapaatalo $\mathrm{H}$. The effect of renal sympathectomy on blood pressure and plasma renin activity in spontaneously hypertensive and normotensvie rats. Acta Physiol Scand $115,289-293,1982$.

Schramm LP, Chornoby ES. Sympathetic activity in spontaneously hypertensive rats after spinal transsection. Am $\mathrm{J}$ physiol 243, R5006-R5111, 1982.

Schramin LP, Livingstone R. Propriospinal and descending systems inhibiting and exciting renal nerve activity in hypertensive and normotensive rats. In: Organization of the autonomic nervous system: central and peripheral mechanisms (eds: Ciriello J, Calaresu FR, Renaud LP, Polosa C). Liss AR Inc, New York, 1987, pp 111-120.

Sealey JE, luatagh JW. How to do a plasma renin assay. Candiol Med 2, 1079$1092,1977$.

Simon OR, Schramm LP. The spinal course and medullary termination of myelinated renal afferents in the rat. Brain Res $290,239-247,1984$.

Smedes F, Kraak JC, Poppe H. Simple and fast solvent extraction system for selective and quantitative isolation of adrenaline, noradrenaline and dopamine from plasma and urine. J Chromatogr 231, 25-39, 1982.

Smits JFM. The antihypertensive effect of propranolol. Thesis, Maastricht, 1980.

Smits JFM, Kasbergen CM, Van Essen H, Kleinjans JC, Struyker-Boudier HAJ. Chronic local infusion into the renal artery of unrestrajned rats. Am $J$ Physiol 24.4, H304-H307, 1983.

Smits JFM, Brody MJ. Activation of afferent renal nerves by intrarenal bradykinin in conscious rats. Am J Physiol 247, R1003-R1008, 1984. 
Smits JFM and struyker-Bowdier HAJ. Systemic and regional hemodynamics following acute inhibition of anglotensin $I$ converting enzyme in the conscious spontaneously hypertensive rat. Prog Pharmacol 5: $39-49$, 1984 .

Smits JFM, Van Essen H, Tijssen CM, Struyker-Boudier HAJ. Effects of ketanserin on hemodynamics and baroreflex effects in conscious spontaneously hypertensive rats. J Cardiovasc Pharmacol 10, 1-8, 1987 .

Smith PG, Poston CW, Mills E. Ontogeny of neural and non-neural contributions to arterial blood pressure in spontaneously hypertensive rats. Hypertension 6, 54-60, 1984 .

sparf $B$. On the turnover of acetylcholine in the brain. Acta Physiol Scand (suppl) $397 \quad 7-47,1973$.

Spitalewitz S, Chou S-Y, Faubert PF, Porush JG. Effects of diuretics on inner medullary hemodynamics in the dog. Circ Res 1982 51, 703-710.

stebbins CL, Smith RC, Langhurst JC. Effect of prostaglandins on bradykinin-induced visceral cardiac reflexes. Am J Physiol 249, B155-H163, 1985.

Stein RD, Weaver LC. Multi- and single-fiber mesenteric and renal sympathetic responses to chemical stimulation of intestinal receptors in cats. J Physiol (Lond) 396, 155-172, 1988.

Stella A, Golin R, Busnardo I, Zanchett 1 A. Effects of afferent renal nerve stimulation on renal hemodynamic and excretory functions. Am J Physiol 247, H576 H583, 1984.

Stella A, Weaver L, Golin R, Genovesi $S$, Zanchetti A. Cardiovascular effects of afferent renal nerves stimulation. Clin Exp Hypert Theor Pract A9 (suppl 1), $97-112,1987$.

Struyker-Boudier HAJ, Renal sodium excretion and blood pressure regulation. In: Natrium und Hypertonie (eds: Bock KD, Schrey A), München, 1981, pp $38-50$.

Struyker-Boudier HAJ, Evenwel RT, Smits JFM, Van Essen H. Baroreflex sensitivity during the development of spontaneous hypertension in rats. Clin Sci $62,589-594,1982$.

Struyker-Boudier HAJ, Smits JFM, Kleinjans JCS, Van Essen H. Hemodynamlc actions of diuretic agents. Clin Exp Hypert AS (2), 209-223, 1983.

Struyker-Boudier HAJ, Vervoort-Peters LHTM, Rousch MJM, Smits JFH, ThHissen FHW. Beta-adrenoceptors in kidney tubules of spontaneously hyperten sive and normotensive rats. Life Sci 38, 137-145, 1985.

struyker-Boudier HAJ. Dopamine receptors in the cardiovascular system and the kidney. Prog Pharmacol 6, 81-96, 1986.

Struyker-Boudier HAJ, Janssen BJA, Smits JFM. Adrenoceptors in the kidney: localization and pharmacology. Clin Exp Hypert Theor Pract A9 (suppl. 1), $135-150,1987$.

Takahashi H, Iyoda I, Yamasaki H, Takeda K, Okajima H, Sasaki S, Yoshimura. $M$, Nakagawa $M$, IJichi $H$. Retardation of the development of hypertension in DOCA salt rats by renal denervation. Jpn Clic J 48, 567-574, 1984 .

Takeda K, Okajima H, Hayashi J, Kawasaki S, Sasaki S, Nakagawa M, Ijlchi H. At tenuation of hypothalamo-sympathetic hyperactivity by renal denervation in experimental hypertensive rats. Clin Exp Eypert Theor Pract A9 (suppl 1), 75-88, 1987 .

Tarazi RC, Fouad FM. Circulatory dynamics in autonomic fallure. In: Autonomic failure (ed: Bannister R). Oxford University Press, 0xford, 1983, pp $96-113$.

Taylor SH. Diuretics in cardiovascular therapy. Persulng the past, practising in the present, preparing for the future. Z Kardiol 74 (suppl 2), $2-12,1985$.

Thorén P, Andresen MC, Brown AlM. Effects of changes in extracellular tonic 
concentrations on artic baroreceptors wh nonnelinated afferent

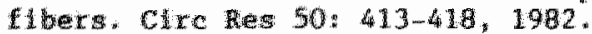

Thoren P. Eferent renal nerve tratic in the spontaneously bypertensive rat. Clin Rwp Hyert Theor Pract A9 (supp 1), 135-150, 1987.

Thurau K. Renal hemodynamics. Am J Med 36, 698-719, 1964.

Tobey 3C, Weaver LC. Prescoreceptor modulation of renal but not splenic sympathetic reflexes. Am I Physiol 252, R26-R33, 1987.

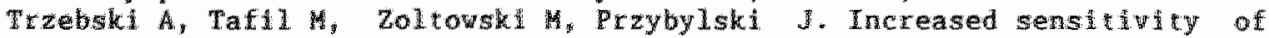
the arterial chemoreceptor drive in young men wth mild hypertension. Cardlovase Res 16, 1630-172, 1982.

Tudke BI, BIntz RC. Effect of furosemide administration on glomerular and wubular dynamies in the rat. Kidney Int $26,112-121,1984$.

Tucke $B J$, Mundy CA, Llantz RC. Adrenergic and angiotensin II infuences on renal vasculat tone in chronle sodium depilion. Am 3 Physiol 252 , F812-1817, 1987.

Tutte RS, Moleary M. Mesenteric baroreceptors. An J Physiol 229, 1514$1519,1975$.

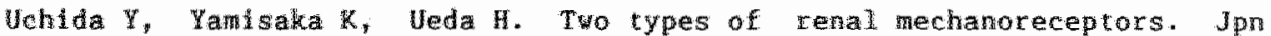
Heart J 1971 12, 233-241, 1971.

Veda $\mathrm{F}$, Uchida $\mathrm{Y}$, Ramisaka $\mathrm{R}$. Mechanisms of the reflex depressor effect by kidney dog. Jpn Heart J 8,597-606, 1967.

Van Brumelen $P$, Man 4 't Veld AJ, Schalekamp MDN. Hemodynamic changes durlng long-tern thiazide treatment of essential hypertension in responders and nonresponders. Clin Pharmacol Ther 27: 328-336, 1980 .

VandenBusse Versteeg DHG, DeJong. Brain dopamine depletion by lesions in the substantla nigra at enuates the development of hypertension in the spontaneously hypertensive rat. Brain Res $368,69-78,1986$.

Varter AJ, Carison $\%$. Mechantsm of the effects of furosemide on renin secretion in anesthetzed dogs. Circ Res 25, 145-152, 1969.

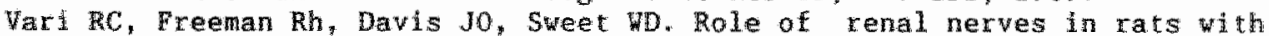
low-sodiun, one-kidney hypertension. Am $\&$ Physiol 250, H189-H194, 1986.

Vari RC, Zinn $S$, Verburg $K$, Freeman RH. Renal nerves and the pathogenesis of anglotensin-1nduced hypertension Hypextension 9, 345-349, 1987.

Verberne AJM, Lewis SJ, Jarrot B, Louls WJ. Medial prefrontal cortical lesions and baroreceptor heart rate reflex sensitivity in the spontaneously hypertensive rat. J Hypert $6,123-127,1988$.

VAlarreal. D, Freeman RH, Davis J0, Garoutte G, Sweet WD. Pathogenesis of one-kidney, one-clip hypertension rats after renal denervation. Am $J$ Phys 101 247, H61-H66, 1984.

Virus RM, Knuepter MM, McManus DR, Brody MJ, Gebhard GF. Capsaicin treatment in adult Wistar-kyoto and spontaneously hypertensive rats: effects on noclceptive behavior and cardiovascular regulation. Eur $y$ Pharmacol $72,209-217,1981$.

Wallenstein $S$, zucker $C L$, Fleis $J L$. Some statistical methods useful in circulation research. Circ Res $47,1-9,1980$.

Weaver LC, Genovesi S, Stella A, Zanchetti A. Neural, hemodynamic and renal responses to stimulation of intestinal receptors. Am $\mathrm{J}$ Physiol 253, H1167-H1176, 1987.

Webb RL, Brody MJ. Functional identification of the central projections of afferent renal nerves. Clin Exp Hypert Theor Pract A9 (suppl 1), 47$58,1987$.

Westfall $T C$. Meldrum MJ. Alterations in the release of norepinephrine at the vascular neuroeffector junction in hypertension. Ann Rev Pharmacol Toxicol $25,621-641,1985$. 
Whitlow PL, Katholi RE. Neurohumoral activity and the role of the renal nerves in canine coarctation of the aorta. Am $J$ Cardiol 49, 888, 1982.

Wilcox CS, Aminoff MJ, Slater JDH. Sodium homeostasis in patients with autonomic fallure. Clin Sci Mol Hed 53, 321-328, 1977.

Wilcox CS. Body fluids and renal function in autonomic fallure. In: Autonomic failure (ed: Bannister R). Oxford University Press, oxford, 1983, pp 115-154.

Villamsion HE, Bourland WA, Marchand GR. Inhlbition of furosemide induced increase in renal blood flow by Indomethacin (38497). Proc soc Exp Biol Med 148, 164-167, 1975 .

Williamson HE, Bourland WA, Marchand GR, Farley DB, van Orden DE. Furosemide induced release of prostaglandin $\mathrm{E}$ to increase renal blood flow (38982). Proc Soc Exp Biol Med 150, 104-106, 1975.

Winternitz SR, Katholi RL, Oparil S. Role of the renal sympathetic nerves in the development and maintenance of hypertension in the spontaneously hypertensive rat. J Clin Invest 66, 971-978, 1980.

Winternitz SR, Katholi RL, Oparil S. Decrease in hypothalamic norepinephrine content following renal denervation in the one-kidney, oneclip Goldblatt hypertensive rat. Hypertension $4,369-373,1982$.

Wyss JM, Aboukarsh N, Oparil S. Sensory denervation of the kidney attenuates renovascular hypertension in the rat. An J Physiol 250, H82H86, 1986.

Yamada S, Ishima $T$, Tomita $T$, Hayashi M, Hayashi $E$. Increased renal betaadrenoceptors in stroke-prone spontaneously hypertensive rats. Eur J Pharmacol 83, 149-150, 1982 .

Yamada S, Nakamoto M, Hayashi M, Tomitta T, Hayashi E. Tubular and glomerular adrenoceptors in stroke-prone spontaneously hypertensive rats. J Hypertension 4 (suppl 3), 209-211, 1986.

Yamori $Y$, Organ differences of catecholamine metabolism in spontaneously hypertensive rats. In: Spontaneous hypertension (ed: Okamoto K), Igaku Shoin, Tokyo, 1972, pp 59-68.

Zambraski EJ, Prosnitz EH, DiBona GF. Lack of evidence for renal vasodilation in anesthetized dogs. Proc Soc Exp Biol Med 158, 462-465, 1978 .

Zanchett A, Stella A, Leonetti G, Morganti A, Terzoli L. Control of renin release. a review of experimental evidence and clinclal implications. Am J Cardiol 37, 675-691, 1976.

Zerbe Go. Randonization analysis of the completely randomized design extended to growth and response curves. J Am Statis Assoc 4, 215-222, 1979.

Zimpfer M, Pit SP, Vatner SF. Effects of anesthesia on the canine carotid chemoreceptar reflex. Circ Res 48, 400-406, 1981. 

Nijmegen P.H.W. van de Kroon en Prof. dx. A.P. van Overbeeke); bijvakken ontwikkelingsbiolagie der dieren (Prof. dr. J.M. Denucé) en Inspanningsfysiologie (Prof. dr. R.A. Binkhorst)

1984 Wetenschappelijk assistent, werbonden aan de vakgroep Farmakologie wan de Rijksuniversiteit Limburg war dit proefschrift werd bewerkt

1988

Post-doktoraal medewerker, verbonden aan de vakgroep Farmakologie van de Rijksuniversiteit Limburg ten behoeve van een onderzoeksprojekt betreffende langdurige antihypertensieve therapie

\section{Publikaties}

Schoots AFM, Jansisen BJA, Denucé JM. Antigenicity of highly purified hatching enzyme from the pike, Esox lucius. Arch Int Physiol Btochem 89, B $75-B 76,1981$

BJA Janssen, H wan Essen, HAJ Struyker Boudier, JFM Smits. The role of renal nerves in the development of hypertension induced by intrarenal noradrenaline lnfusions. Naunyn Schmiedeberg's Arch Pharmacol 330 (suppi) $R 48,1985$

BJA Janssen, HAJ Struyker Bowdier, JFH Smits. Dorsal spinal rhizotomy of renal afferent nerves prevents the reflex effects induced by intrarenal bradykinin infusion in the rat. Pharmaceut Whbl Sci Ed 7, 231, 1985

JLML le Moble, BJA Janssen, RW Lappe, MJ Brody, HAJ Struyker Boudier, JFM Smits. Pharmacological evidence for rapid destruction of efferent renal nerves in rats by intrarenal infusion of 6-hydroxydopamine. J Hypertension 3 (suppl 3), S137-5140, 1985

A Struyker Boudier, J Smits, H Nievelstein, J le Noble, B Janssen. Antihypertensive drugs: renal sites of action. Naunyn Schmjedeberg"s Arch 
Pharmacol 330 (suppi) $\mathbb{R} 4,1985$

BJA Janssen, $H$ wan Essen HAJ Struyker Boudier, JFM Smits. Role of sensory nerves in hypertension induced by intrarenal norepinephrine infusions. Pharmaceut Wkbl Sci Ed 8, 273, 1986.

H Van Essen, B Janssen, H Struyker Boudier, J Smits. Hypertension induced by intrarenal norepinephrine infusions: role of afferent renal nerves: (ARN). Fed Proc 46, 669, 1987.

BJA Janssen, JJM Debets, HAJ Struyker Boudier, JFM Smits. Role of sensory renal nerves in the dewelopment of spontaneous hypertension in rats. Clin Exp Hypertension A9 (suppl), 227-239, 1987

B Janssen, H van Essen, H Struyker Boudier, J Smits. Acute regional hemodynamic effects of furosemide in rats: role of afferent renal nerves. Pharmaceut Wkbl Scil Ed 10, 294, 1987.

B Janssen, H Struyker Boudier, J Smits. Role of afferent renal nerves (ARH) in spontaneous hypertension in rats. Fed Proc 46, 669, 1987

BJA Janssen, HAJ Struyker Boudier, JFM Smits. Do afferent renal nerwes play an important rale in the development of spontaneous hypertension in rats. J Hypertension 5 (suppl) S375-5377, 1987

JFM Smits, JCS Kleinjans, BJA Janssen, HAJ Struyker Boudier. Characterization of hypertension induced by long-term intratenal morepinephrine infusion in conscious rats. Clin Exp Hypert A9 (suppl), 197-209, 1987

HAJ Struyker Boudier, BJA Janssen, JFM Smits. Adrenoceptors in the kidney: localization and pharmacology. Clin Exp Hypert A9 (suppl), 135 150, 1987

HAJ Struyker Boudier, HMNM Nievelstein, BJA Janssen, JFM Smits . Baroreflex control of the circulation in hypertension and aging. In: Early pathogenesis of primary hypertension (eds A Hofman, DE Grobbee, MADH Schalekamp). Elsevier Science Publishers BV, Amsterdam, 157-165, 1987

BJA Janssen, $H$ van Essen. Furosemide induces acute renal vasoconstriction in conscious rats. Naunyn Schmledeberg's Arch Pharmacol 337 (suppl), R50, 1988

B Janssen, H van Essen, J Smits, H Struyker Boudier. Comparison of hemodynamic effects evoked by intrarenal and intramesenteric infusions of bradykinin and adenosine in conscious rats. FASEB $J 2, A 502,1988$

BJA Janssen, JFM Smits. Renal nerves in hypertension. Mineral Electrolyt. Metab, 1988 (in press)

B.JA Janssen, H van Essen, HAJ Struyker Boudier, JFM Smits. Hemodynamic effects of activation of renal and mesenteric sensory nerves in consclous unrestrained rats (submitted)

BJA Janssen, H van Essen, HTM Vervoort-Peters, HAJ Struyker Boudier, JFM Smits. Effects of renal sensory denervation on sympathetic function and baroreflex sensitivity in spontaneously hypertensive rats (submitted)

BJA Janssen, H van Essen, LHTM Vervoort-Peters, HHW Thifssen, FHM Derkx, HAJ Struyker Boudier, JFM Smits. Effects of complete renal denervation and selective afferent remal denervation on the hypertension induced by intrarenal norepinephrine infusions in conscious rats (submitted)

BJA Janssen, HAJ Struyker Boudier, JFM Smits. Acute arteriolar vasoconstriction following furosemide in conscious spontaneously hypertensive rats (submitted)

BJA Janssen, H van Essen, HAJ Struyker Boudier, JFM Smits. Acute renal. vasoconstrictor response to furosemide in normotensive rats: its fhechanism of action (in preparation)

BJA Janssen, H van Essen, HAJ Struyker Boudier, JFM Smits. Role of afferent renal nerves in renal sodium excretion (in preparation) 